\begin{tabular}{|c|c|}
\hline Title & Surfactant adsorption to soil components and soils \\
\hline Author(s) & Ishiguro, Munehide; Koopal, Luuk K. \\
\hline Citation & $\begin{array}{l}\text { A dvances in colloid and interface science, 231, 59-102 } \\
\text { https://doi.org/10.1016/.cis.2016.01.006 }\end{array}$ \\
\hline Issue Date & 2016-05 \\
\hline Doc URL & http:/hdl.handle.net/2115/70018 \\
\hline Rights & $\begin{array}{l}\text { O2016, Elsevier. Licensed under the Creative Commons A ttribution-NonCommercial-NoDerivatives } 4.0 \text { International } \\
\text { http://creativecommons.org/icenses/oy-nc-nd/4.0/ }\end{array}$ \\
\hline Rights(URL) & http://treativecommons.org/icenses/by-nc-nd/4.0/ \\
\hline Type & article (author version) \\
\hline File Information & A dv Colloid Interface Sci231_59-102.pdf \\
\hline
\end{tabular}

Instructions for use 


\section{Surfactant adsorption to soil components and soils}

Munehide Ishiguro $^{1 *}$ and Luuk K. Koopal ${ }^{2 \#}$

${ }^{1}$ Research Faculty of Agriculture, Hokkaido University, Sapporo, Japan.

${ }^{2}$ Physical Chemistry and Soft Matter, Wageningen University and Research, Wageningen, The Netherlands.

Corresponding author. Tel.: +81 11706 2565; fax.: +81 117062494 .

E-mail address: ishi-m@env.agr.hokudai.ac.jp

${ }^{\#}$ Visiting professor, Research Faculty of Agriculture, Hokkaido University, Sapporo, Japan, May-July 2015. 


\section{ABSTRACT}

Soils are complex and widely varying mixtures of organic matter and inorganic materials; adsorption of surfactants to soils is therefore related to the soil composition. We first discuss the properties of surfactants, including the critical micelle concentration (CMC) and surfactant adsorption on water/air interfaces, the latter gives an impression of surfactant adsorption to a hydrophobic surface and illustrates the importance of the CMC for the adsorption process. Then attention is paid to the most important types of soil particles: humic and fulvic acids, silica, metal oxides and layered aluminosilicates. Information is provided on their structure, surface properties and primary (proton) charge characteristics, which are all important for surfactant binding. Subsequently, the adsorption of different types of surfactants on these individual soil components is discussed in detail, based on mainly experimental results and considering the specific (chemical) and electrostatic interactions, with hydrophobic attraction as an important component of the specific interactions. Adsorption models that can describe the features semi-quantitatively are briefly discussed. In the last part of the paper some trends of surfactant adsorption on soils are briefly discussed together with some complications that may occur and finally the consequences of surfactant adsorption for soil colloidal stability and permeability are considered. When we seek to understand the fate of surfactants in soil and aqueous environments, the hydrophobicity and charge density of the soil or soil particles, must be considered together with the structure, hydrophobicity and charge of the surfactants, because these factors affect the adsorption. The $\mathrm{pH}$ and ionic strength are important parameters with respect to the charge density of the particles. As surfactant adsorption influences soil structure and permeability, insight in surfactant adsorption to soil particles is useful for good soil management. 
Keywords: humic substances, silica, metal (hydr)oxides, kaolinite, montmorillonite, soil components

\section{Contents}

1. Introduction

2. Surfactants and their characteristics

2.1. Surfactant types

2.2. Surfactant adsorption at water/air surfaces

2.3. Micelles and their shape

3. Soil components

3.1. Humic substances

3.2. Silica and metal (hydr)oxides

3.3. Silicate clays (alumino-silicates)

3.4. Concluding remarks

4. Surfactant binding to humic substances

4.1. Early experimental studies and isotherms measured with surfactant electrodes

4.2. Surfactant binding measured by solid-phase micro-extraction

4.3. Modeling surfactant - humic substance interaction

4.4. Sorption of organic cations and risk assessment

5. Surfactant adsorption to metal (hydr)oxides and silicas

5.1. General aspects and reviews

5.2. Surfactant adsorption and charge regulation

5.3. Ionic surfactant adsorption on rutile.

5.4. Ionic surfactant adsorption on silica

5.5. Nonionic surfactant adsorption on silica

6. Surfactant adsorption to silicate clays (alumino-silicates) 
6.1. General aspects and outline

6.2. Cationic Surfactant adsorption on kaolinite

6.3. Anionic surfactant adsorption on kaolinite

6.4. Nonionic surfactant adsorption on kaolinite

6.5. Anionic surfactant adsorption on montmorillonite

6.6. Cationic surfactant adsorption on montmorillonite

6.7. Nonionic surfactant adsorption on montmorillonite

7. Surfactant adsorption modeling

7.1 General remarks

7.2 Mean-field models of monocomponent solute adsorption

7.3 Aggregation models for monocomponent solute adsorption

7.4 SCFA model for surfactant adsorption

7.5 NICA-Donnan model applied to surfactant sorption on humic and fulvic acids

8. Surfactant adsorption on soils

8.1. General considerations and trends

8.2. Fate of surfactants in soils; degradation, hysteresis and precipitation

8.3. Influence of surfactant adsorption on soil structure and permeability

Acknowledgements

References 


\section{Introduction}

(1)

Surfactants have an amphiphilic character, which means that they are composed of a polar (hydrophilic) and an apolar (hydrophobic) part. A fundamental property of surfactants in solution is their ability to form micelles (colloidal-sized surfactant aggregates) at a characteristic surfactant concentration, the critical micelle concentration or CMC. At the CMC the surfactant monomers aggregate into micelles with the hydrophobic tail groups located in the core of the micelle and the hydrophilic head groups in the aqueous interface. In this way the unfavourable contacts of water with the apolar tails are minimized. Micellization gives surfactants their excellent detergency and solubilization properties; due to solubilization (partially) hydrophobic organic compounds dissolve in a micellar solution much better than in water alone. Another characteristic feature of surfactants is their tendency to adsorb at interfaces, mostly in an oriented fashion. The name surfactant is derived from this property; it is a contraction of surface-active agent. By surfactant adsorption the particle surface characteristics change; for instance, by adsorbing with their hydrophobic part to a hydrophobic surface the hydrophilic part protrudes in solution and makes the particle surface hydrophilic, which facilitates wetting and dispersion of the particles in aqueous solutions [1]. Due to their specific character surfactants are widely used chemicals. In household applications they are used in detergents, soaps and shampoos. In industrial applications surfactants are used for emulsification, dispersion, flocculation, wettability, flotation, foaming and so on [2]. In agricultural applications, surfactants are used for the formulation of hydrophobic agricultural chemicals to make these chemicals easily dispersible in water and as anticaking agents for chemical fertilizers [3]. In some cases surfactants are used in soil and groundwater remediation $[4,5]$. The disadvantage of the application of large amounts of surfactants worldwide (>15 Mton/year) [6], is that their 
discharge is a cause of water pollution [7] and from the aqueous environment surfactants can easily bind to soil components. Dissolved surfactants are considered contaminants in aqueous environments and, in principle, they should be removed from wastewater before entering receiving waters. The 'Handbook of Detergents, Part B, Environmental impact' [8] considers the environmental aspects of most surfactant types. Recent information on the aquatic toxicity, biodegradability, and bioaccumulation, which are relevant for the assessment of surfactant ecotoxicity, can be found in [9-13]. Rebello et al. [10] conclude in their review: “...regarding surfactants as non-pollutants is a mistake. Visible manifestations of surfactant toxicity are available in the case of microbes, plants and animals”.

Soils are complex materials; they contain both inorganic particles and organic matter, see e.g.[14]. Roughly speaking, inorganic particles can be divided into primary minerals (physically weathered minerals from rocks), silicas, metal (hydr)oxides and layered aluminosilicates (clays) and within these three groups the particle sizes can vary largely. In general the primary minerals and silica particles make-up the sand and silt fraction, metal (hydr)oxides and aluminosilicates contain the smaller particle size fractions with large specific surface areas. All these soil particles contain in the normal $\mathrm{pH}$ range a surface charge, depending on the nature of the particles, which is largely or partly variable by changing the $\mathrm{pH}$. This primary charge is due to the adsorption of protons and/or hydroxyl ions to functional groups (sites) present at the surface of the particles. Soil organic matter, which potentially acts as sorbent, originates from living organisms. It consists of structurally randomized macromolecular residues due to partial degradation, rearrangement and recombination of the original organic structures [15]. Mostly a distinction is made between non-humic and humic substances. Non-humic substances are chemically recognizable in biochemistry and are transitory in soil. Humic substances are not easily degradable and carry specific functional groups that can dissociate depending on the 
51

structure of the group. As most groups are acidic the humic substances also carry a charge that is variable with $\mathrm{pH}$.

The nature of the surface sites of the inorganic particles and the functional groups of the organic particles, determine the particle charge and chemistry and charge govern to a large extent the sorption of other components, including surfactants, to the particles. Important for surfactant binding are the precise sites present at the surface as they determine the chemical nature of the surface and the primary particle charge. The chemical nature determines the "specific" or "chemical" interactions with the polar and apolar parts of the surfactant molecules and the charges determine the electrostatic interaction, which can be either attractive or repulsive, depending on the charge signs of surfactant and particles. Together these interactions determine the mode of the surfactant adsorption to the particles (with their hydrophilic or their hydrophobic part directed to the surface). As far as we are aware, there is no comprehensive review on surfactant sorption to soils, most likely this is due to the complicated and diverse nature of the soils and the many different types of surfactants.

In the present paper first the properties of surfactants are considered in some detail, including surfactant adsorption on the air/water interface, which can illustrate the importance of the CMC for the adsorption process and gives an impression of surfactant adsorption to a hydrophobic surface. Surfactant-soil interactions are determined by the soil components; therefore attention is given to the different types of soil particles: humic substances, silica and metal oxides and aluminosilicates. Information is provided on their structure, surface properties and primary charge characteristics, which are all important for surfactant binding. Subsequently, the adsorption of different types of surfactants on these individual soil components is discussed in detail, based on mainly experimental results and considering the various specific (chemical) and electrostatic interactions. Adsorption models that can describe the features semi-quantitatively are explained and discussed. In the last 
part of the paper some trends of surfactant adsorption on soils are discussed together with some complications that may occur and finally the consequences of surfactant adsorption for soil colloidal stability and permeability are considered.

\section{Surfactants and their characteristics}

\subsection{Surfactant types}

The chemistry of the polar and apolar part of surfactants can be quite different for different surfactants and based on the nature of the polar head group surfactants are classified into anionics, cationics, nonionics, and zwitterionics. The principal surfactant demand is composed of four types of surfactants: linear alkylbenzene sulfonates (LAS), fatty alcohol ethoxylates (AE), fatty alcohol ether sulfates (FES) and fatty alcohol sulfates (FAS). Both Western Europe and the US rely on the alcohol derivatives for $60-70 \%$ of the major surfactants but in the rest of the world it is $15-30 \%$, globally LAS is the most used surfactant [16]. Next to synthetic surfactants also natural surfactants and biosurfactants exist. Some examples of synthetic and natural surfactants are depicted in Fig. 1.

Biosurfactants are surfactants that are produced extracellularly or as part of the cell membrane by bacteria, yeasts and fungi. Mulligan [17] and Bustamante et al. [18] give examples of biosurfactants and their structures and review the environmental applications for soil remediation and water treatment. Biosurfactant applications in the environmental industries are promising due to their biodegradability, low toxicity and effectiveness in enhancing biodegradation and solubilization of low solubility compounds.

\subsection{Surfactant adsorption at water/air surfaces}

The attraction of surfactants for aqueous solution/air surfaces is due to hydrophobic attraction. Hydrophobic attraction is the thermodynamically favorable tendency of the 
103 surfactant hydrocarbon moiety to escape from the aqueous environment to an apolar

104 environment (air). With adsorption the hydrophobic attraction is the difference between the

105 Gibbs energy (energetic and entropic contributions) of the surfactant tails in aqueous

106 solution and that in the adsorbed state. The adsorption of surfactants at liquid interfaces is

107 generally studied by measuring the surface tension, $\gamma$, and using the Gibbs equation to

108 obtain the adsorption $[19,20]$.

109

$$
\mathrm{d} \gamma=-s_{a}^{(1)} \mathrm{d} T-\sum_{i=2} \Gamma_{i}^{(1)} \mathrm{d} \mu_{i}
$$

110 where $s_{a}^{(1)}$ is the relative surface entropy per unit area, $T$ the absolute temperature, $\Gamma_{i}^{(1)}$ is the

111 relative adsorption of component $i$ and $\mu_{i}$ is the chemical potential of $i$. The quantities $s_{a}^{(1)}$

112 and $\Gamma_{i}^{(1)}$ are conveniently determined by assuming that the solvent (component 1)

113 adsorption is zero $\left(\Gamma_{1}=0\right)$. In the case of surfactant adsorption from ideally dilute aqueous

114 solutions the Gibbs equation can be approximated as [21-23]:

115

$$
\mathrm{d} \gamma=-p R T \Gamma_{\text {suffactant }} \mathrm{d} \ln c_{\text {suffactant }}
$$

116 where $p=1$ for nonionic surfactants (they are only affected by the presence of electrolytes when the electrolyte changes the solvent quality), but for ionic surfactants

$$
p=1+\frac{c_{\text {surfactant }}}{c_{\text {surfactant }}+c_{\text {salt }}}
$$

119 This notation of the Gibbs equation has used the equality $\mathrm{d} \mu_{i}=R T \mathrm{~d} \ln c_{i}$ for all components 120 present and the implicit assumptions are made that the ion concentrations from the

121 background electrolyte are constant, that $T$ is constant, that the adsorption, $\Gamma_{\text {surfactant }}$, is a

122 simplified notation for $\Gamma_{i}^{(1)}$ and that for ionic surfactants the surfactant co-ion adsorption

123 can be neglected. The latter assumption holds best for highly charged surfaces, i.e. at

124 sufficient surfactant ion adsorption. Schematically the effect of surfactants on the surface

125 tension of aqueous solutions is shown in Fig. 2. 
In Fig. 2a $\gamma(\ln c)$ curve is shown, firstly the surface tension progressively decreases with increasing surfactant concentration (region I). In region II the decrease becomes approximately linear and at the start of region III the critical micelle concentration or CMC is reached. Typically, micelles are clusters of 50-200 surfactant molecules. For surfactant concentrations somewhat above the CMC (region III) the monomer concentration stays about constant and so does the chemical potential of the monomeric surfactant. With the Gibbs equation the adsorption, $\Gamma$, can be calculated from the derivative of the $\gamma(\ln c)$ curve. A typical result is depicted in Fig. 2b. The adsorption first increases but in region II, where

134 the slope of the $\gamma(\ln c)$ curve becomes constant (within experimental error), the adsorption

135 reaches a plateau value. In general, the maximum adsorption depends on the effective size 136 of the hydrated head group. In region III the monomer concentration remains constant. Application of the Gibbs equation in region III is not possible because the monomer surfactant concentration stays equal to the CMC, but there is general consensus that the adsorption stays at the level reached at the CMC. For ionic surfactants the $\chi(\ln c)$ curve depends on the ionic strength, see Fig. 3. For the calculation of the adsorption with the

141 Gibbs equation the factor $p$ comes into play, the extreme situations are $p=2$ in the absence of 142 salt and $p=1$ when the salt concentration is much larger than the surfactant concentration.

143 For the intermediate situations $p$ has to be calculated and can gradually increase with 144 increasing surfactant concentration. The maximum attainable adsorption, $\Gamma^{\mathrm{m}}$, depends on 145 the salt concentration and increases with increasing ionic strength. This is due to a decrease 146 in electrostatic repulsion between the surfactant head groups, which reduces the effective 147 head group size. The order of magnitude of adsorption of a univalent surfactant is 1-4 $148 \mu \mathrm{mol} / \mathrm{m}^{2}$ [24], which corresponds to about $0.1-0.4 \mathrm{C} / \mathrm{m}^{2}$ and indicates that the adsorption 149 occurs (due to hydrophobic attraction) even at a considerable electrostatic repulsion 150 between the surfactant ions. 
152

153

154

155

156

157

158

159

160

161

162

163

164

165

166

167

168

169

170

171

172

173

174

175

176

177

\subsection{Micelles and their shape}

The presence of micelles at surfactant concentrations above the CMC is a very important property of surfactants. Micelles are governed by energetic and geometric considerations. A comprehensive review on the models that describe micellization can be found in [25]. In a micelle the hydrophobic attraction between the hydrophobic moieties is just balanced by the repulsion between the hydrated head groups. The hydrophobic attraction is in this case the difference between the Gibbs energy of the surfactant tails in the aqueous solution and that in the core of the micelles. The shape of the micelles depends on the geometric characteristics of the surfactant molecules. In general, the concentration at which the CMC occurs depends on the surfactant structure and solution conditions. With respect to the surfactant structure the variation in the length of the hydrophobic tail of the surfactant is the most important factor and, in general, the CMC decreases as the hydrophobic character of the surfactant increases, see Fig. 2c. For nonionics also the head group size is important; in general the CMC and the surface tension at the CMC increases with the head group size, see Fig. 2d.

The solution conditions affect the CMC of nonionic surfactants when the solvent quality for the head groups is affected. For ionic surfactants the ionic strength and type of surfactant counterion are important. By way of illustration, Fig. 3 shows how the $\mathrm{NaCl}$ concentration affects the surface tension of an ionic surfactant: sodium dodecyl pyridinium chloride (C12PC). It is obvious that the salt concentration changes both the CMC and the slope of the curves. The presence of electrolytes in solution reduces the CMC by shielding the electrostatic repulsion between the ionic head groups. In soils, the CMC of ionic surfactants is likely to be reduced due to the salts present in the soil-solution.

The above illustrates that the CMC of surfactants in aqueous solution varies with surfactant structure and presence of electrolyte in the case of ionic surfactants; the presence of various organic compounds and the temperature also affect the CMC. Although the CMC of a surfactant may vary under different conditions, the CMC is a characteristic property of a 
178 surfactant. Tabulated values of many surfactants (for ionic surfactants with specification of

179 the salt concentration or ionic strength) can be found in [24, 26, 27].

180 The shape of the micelles varies from surfactant to surfactant and for ionic surfactants

181 the salt concentration is also affecting the micelle shape. In general, the micelle shape

182 depends on the area of the apolar tail, $V_{o} / l_{0}$, where $V_{o}$ is the tail volume in the micelle and $l_{o}$

183 the extended tail length, and the equilibrium area, $a_{e}$, of the polar/ionic head group at the

184 micelle surface. The parameter $V_{o}$ varies with the number of hydrophobic groups, chain

185 unsaturation, chain branching and chain penetration by other compatible hydrophobic

186 groups, while $a_{e}$ is mainly governed by electrostatic interactions and head group hydration.

187 For ionic surfactants $a_{e}$ depends on the ionic strength and for large tails the tail packing in

188 the micelle is non-uniform and affects also the equilibrium head group area [28, 29]. The

189 ratio tail area over head group area, $V_{o} / a_{e} l_{o}$, is called the "critical packing parameter" or

190 CPP and the CPP value provides a good indication of the shape of the micelles [30-32]. In

191 aqueous media and $0<\mathrm{CCP}<0.3$ the micelles are spherical (single chained surfactants with

192 large head group areas), for $0.3<\mathrm{CCP}<0.5$ the micelles are cylindrical (single chained

193 surfactants with small head group areas) and for $0.5<\mathrm{CCP}<1$ curved bilayers (lamellar) are

194 formed (double chained surfactant with large head group areas) and for $\mathrm{CCP}=1$ flat bilayers

195 (double chained surfactant with small head group areas). For CCP $>1$ only inverted micelles

196 can exist. When the surfactant concentration is raised above the CMC, linear growth of

197 micelles is the dominating type of growth. Some knowledge about the shape of the micelles

198 is also important because in the adsorbed state surfactants already tend to form micellar type 199 aggregates before the CMC is reached.

\section{Soil components}


The soil organic fraction contains the non-humic substances (e.g., proteins and natural macromolecules) and the humic substances. The humic substances are divided into fulvic acid, humic acid and humin by their aqueous solubility; humic and fulvic acids are soluble in the normal $\mathrm{pH}$ range and humins are not. Fulvic and humic acids are distinguished by their solubility at low $\mathrm{pH}$ : fulvic acids are soluble in all $\mathrm{pH}$, whereas humic acids precipitate at low pH. For the isolation and separation of the humic substances the procedure described by Swift et al. [33] is mostly used as the International Humic Substances Society recommends it.

Humic particles found in soil, sediment and water have an extreme structural complexity. In an overview Leenheer [34] discusses the successive approximations of model structures 214 to molecular structures that have appeared in literature for dissolved humic particles. 215 Present days insight is that the old "polyelectrolyte model” that represented the dissolved humic particles as randomly coiled macromolecules resulting from various chemical condensation and coupling reactions of smaller biomolecular constituents is no longer adequate. New structural models view dissolved humic particles as dynamic associations of relatively low molar mass components stabilized by hydrophobic interactions and hydrogen bonds [35] or as complex supramolecular assemblies of small molecules, oligomers, and 221 polymers aggregated into supramolecular assemblies by non-covalent forces [36].

From physicochemical point of view the dynamic character of the aggregation [37], the 223 presence of a diversity of functional groups that protrude in the aqueous phase [38] and the 224 fact that the aggregates have an open structure [39] that allows the aqueous solution to 225 partly permeate through the particles has to be taken into account. The functional groups can dissociate or associate with protons and this causes the primary charge of the particles that governs the electrostatic interactions. With physicochemical treatments humic particles 228 should therefore be considered as amorphous, polydisperse, chemically complex and 229 internally structured supramolecular particles that are held together by hydrophobic 
230 attraction and hydrogen bonding and that are partly permeable by the aqueous solution.

231 Through their structure and dissociation or association of the functional groups with protons 232 the particles have in aqueous solutions a polyelectrolyte nature that can be classified as 233 soft-colloidal matter and both their charging and electrokinetic behavior [40] are different 234 from that of rigid solid particles. Due to their 'open structure' humic and fulvic acid have 235 very large sorption capacities per kg of material. The main differences between humic and 236 fulvic acids are their functional group density, average molar mass and hydrophobicity: 237 fulvic acids have a relatively low molar mass, a relatively high functional group density and 238 a relatively low hydrophobicity. Humin particles are only poorly investigated, but based on 239 the isolation procedure it may be expected that they are solid like (much less permeable 240 than humic acid) with a relatively low functional group density, have a large particle mass 241 and are hydrophobic in nature.

242 For a further physicochemical characterization of humic and fulvic acids the proton 243 binding characteristics of humic and fulvic acids are relevant. The heterogeneity of the 244 proton binding groups of humic substances is considerable with, in general, two main 245 classes of groups each with a wide distribution [41, 42]. The low proton affinity groups are 246 mostly referred to as 'carboxylic' groups ( $\mathrm{pK}_{\mathrm{H}}$ around 3-4) and the high proton affinity 247 groups as 'phenolic groups' $\left(\mathrm{pK}_{\mathrm{H}}\right.$ around 8-9). For the $\mathrm{pH}$ conditions in natural waters 248 humic and fulvic acid are negatively charged and $\mathrm{pH}$ and ionic strength affect the proton 249 charge density [43], the hydrodynamic radius of the humic particles [44] and their 250 electrokinetic behavior [40]. Fig. 4 gives a typical example of proton binding to a humic 251 acid at four different salt concentrations [45].

252 Much attention has been paid to modeling proton (and heavy metal ion) binding to humic 253 and fulvic acids and the literature is reviewed in [46-50]. In general, models should (i) take 254 explicitly into account the heterogeneity of the functional groups of humic and fulvic acids, 255 (ii) distinguish between electrostatic and ion-specific binding and (iii) account for 
competition for binding among ions. Models that confirm to these conditions are based on

257 intrinsic parameters that are independent of the environmental conditions (e.g., pH, ionic 258 strength). The two most successful models that suit the above conditions are Tipping's 259 ion-binding Model VI/VII [51-53] and the NICA-Donnan model [38, 43, 54-56]. These 260 models can be applied across a wide range of environmental conditions and be used to make 261 predictions outside the calibration range. The heterogeneity of the humic substances is 262 described in Model VI/VII with a wide discrete distribution of (intrinsic) proton affinity 263 constants $[47,51]$ and in the NICA-Donnan model with a bi-modal continuous distribution 264 [57, 58]. For the electrostatic interactions a simple Donnan model is used in the 265 NICA-Donnan model [54] and recently also in Tipping's model [52, 53]. The Donnan 266 models describe the electrostatic interaction with only one average smeared-out electrostatic 267 Donnan potential at given environmental conditions [59]. The Donnan model leads to 268 calculated Donnan potentials that are highly non-Nernstian, see [54]. This means that the 269 Donnan potential is non-linear with a change in $\mathrm{pH}$ and that its value is also strongly ionic 270 strength dependent. By considering a great deal of the existing data up to 2003, Milne et al. $271[55,56]$ have been able to establish two sets of parameters that can describe the proton and 272 other ion binding to, respectively, generic-HA and generic-FA. These generic-HS 273 compounds can be used as substitutes when no detailed information is present on a specific 274 HS to calculate the ion binding for many ions.

\subsection{Silica and metal (hydr)oxides}

277 In many parts of the world, silica $\left(\mathrm{SiO}_{2}\right)$ is the major constituent of sand and silt. The 278 fine silica particles $(<2 \mu \mathrm{m})$ are classified as clay. Oxides of iron and aluminum are most 279 prominent in soils of the tropics but are widespread elsewhere. Silica has a number of 280 distinct crystalline forms in addition to amorphous forms. Amongst the various 281 crystallographic forms of silica, $\alpha$-quartz is the most abundant, as it is the most stable 
282

crystalline silica phase. Thus, $\alpha$-quartz has been widely used as a model for the investigations of water/silica interface systems in environmental processes. Due to their coarse size, quartz particles have a low specific surface area and do not have colloidal properties, yet they exhibit adsorption. In contrast, amorphous silicas can be very fine and mostly have a large specific surface area. Also because their industrial uses, the adsorption properties of amorphous silicas (mostly synthetic) have attracted much attention.

The surface structure of silica is composed of siloxane sites (-Si-O-Si-) and silanol (-SiOH) sites. Siloxane sites are hydrophobic and silanol sites are hydrophilic; therefore, the higher the silanol content is, the larger is the hydrophilicity of the surface. The silanol groups can be subdivided into isolated (or single), geminal $\left(-\mathrm{Si}(\mathrm{OH})_{2}\right)$ and vicinal or bridged silanols. The vicinal $\mathrm{OH}$ groups are two nearest neighbors that are bound together by a hydrogen bond. The silanol sites are formed by hydroxylation of siloxane bridges. Based on dehydration studies, Zhuravlev [60] has shown that amorphous silica surfaces will contain predominantly single and geminal silanol groups and siloxane bridges, the relative content of each type of group depending on the temperature of dehydration and the subsequent rehydration conditions. Direct information on the distribution of silanol groups over isolated, geminal and vicinal groups can be obtained from NMR studies. Chuang and Maciel [61] have reported that the surface of amorphous silica powders have a roughly equal distribution of isolated and geminal silanols. Yang and Wang [62], who reviewed computational simulations, conclude that the normal siloxane bond is hard to be hydrolyzed because of the high reaction barrier, but siloxane surface structures with a large strain stress and surface defects are rather readily broken by water attack, especially with the aid of extra water molecules. The presence of silanols next to siloxane groups thus also follows from the simulation studies.

From hydration-dehydration and tritium and deuterium exchange studies it can be concluded that when an amorphous silica surface is hydroxylated to the maximum state the 
308 silanol density is around 4.9 groups per $\mathrm{nm}^{2}$ independent on the history of the sample [60].

309 This number is about two times smaller than the classical value of number of silanols of about 8 groups per $\mathrm{nm}^{2}$ derived by Iler using the assumption that for each surface Si there is one $\mathrm{OH}$ group [63]. The difference can be explained by the fact that only half of the Si atoms are capable of holding $\mathrm{OH}$ groups [64]. Tamura et al. [65] give an overview of hydroxyl site densities of silicas and a range of metal oxides (Al, Fe, Mn, Ti, Sn, Zn, Mg).

314 From the collected densities it follows that the hydroxyl content of silica is substantially lower than that of the metal oxide surfaces. The high hydroxyl content of the metal oxides explains that these surfaces are more hydrophilic than the silica surface.

The sites responsible for the charging of the aqueous interface of silica and metal oxides 318 can be derived from the mineral structure. For crystalline minerals the structure is well 319 known through X-ray diffraction measurements and in combination with electron 320 microscopy also the dominant crystal faces that make up the surface can be detected. Using 321 this information in combination with Pauling's bond valence concept [66] and its refinement 322 [67-69] the protonation properties of the oxygen containing surface groups can be derived. 323 In the bond valence concept, the charge of the central cation is distributed over the 324 surrounding O-ligands and the sum of bond valences around oxygen should be equal to the 325 oxygen valence. Hiemstra et al. [70, 71] have used the bond valence concept in the 326 multi-site complexation (MUSIC) model to describe the protonation of mineral surfaces. 327 The model allows differentiating various types of surface groups in terms of formal charge 328 and takes into account that most metal oxide surfaces have multiple types of surface oxygen 329 groups that can react with protons. The $\mathrm{pK}_{\mathrm{H}}$ values of the groups can be predicted and the 330 model has highlighted that the two $\mathrm{pK}_{\mathrm{H}}$ values of two successive protonation steps on one 331 and the same O-ligand differ by about 10 pK-units $\left(\Delta \log K_{H} \approx 10\right)$. This implies that 332 practically only one proton association or dissociation step per surface group can occur in 333 the normal $\mathrm{pH}$ window. Other predictions are: (i) the inertness of double coordinated 
surface groups on sesquioxides, which may result in non-charged perfect crystal faces for gibbsite [72, 73] and hematite [73, 74], and (ii) the difference in proton affinity for the two basic types of triply coordinated groups of goethite [71]. The MUSIC model may also account for changes in temperature as shown by Machesky et al. [75]. It should further be noted that the predicted $\Delta \mathrm{pK}_{\mathrm{H}}$ value of 10 provides evidence that the older description of the proton charging of mineral surfaces based on two protonation steps for each surface group ( $\equiv \mathrm{SO}^{-}$to $\equiv \mathrm{SOH}^{0}$ to $\equiv \mathrm{SOH}_{2}^{+} ; 2-\mathrm{pK}$ model) is fundamentally wrong.

It is instructive to discuss the primitive, but relevant, situation of a homogeneous silica and gibbsite surface; each containing singly coordinated surface hydroxyls only [76-78]. According to the bond valence concept, the silanol group has a formal charge of zero $\left(\equiv \mathrm{SiOH}^{0}\right)$, while the aluminol group has a formal charge of $-1 / 2\left(\equiv \mathrm{AlOH}^{-1 / 2}\right)$. By varying the $\mathrm{pH}$ of the aqueous solution the $\equiv \mathrm{SiOH}^{0}$ can release its proton and becomes $\equiv \mathrm{SiO}^{-1}$, while the $\equiv \mathrm{AlOH}^{-1 / 2}$ group may associate with a proton to form $\equiv \mathrm{AlOH}_{2}{ }^{+1 / 2}$; therefore, the silica surface is acidic and the gibbsite surface is amphoteric. This difference leads to a different type of point of zero net proton charge (pznpc): the silica charge approaches the pznpc asymptotically (all groups in the protonated form at low $\mathrm{pH}$ ), while the charge of gibbsite changes linearly around the pznpc (equal numbers of $\equiv \mathrm{AlOH}^{-1 / 2}$ and $\equiv \mathrm{AlOH}_{2}^{+1 / 2}$ ). To be able to calculate the charge vs. $\mathrm{pH}$ curves at different ionic strength a further model is required that describes the electrical double layer. For flat surfaces the diffuse part of the electrical double layer can be described with the Debye-Hückel or the Gouy-Chapman model [79], but for a good description of the electrical double layer of silica, gibbsite and other mineral surfaces it is necessary to incorporate besides the diffuse layer also a Stern layer adjacent to the surface. The Stern layer accounts for the dimensions of the counterions close to the surface and allows the structure of water near the mineral surface to be different from bulk water [80]. The MUSIC model in combination with the double layer model provides the means to calculate the proton charge $\mathrm{pH}$ curves of silica and gibbsite. As a 
360

361

362

363

364

365

366

367

consequence of protonation reactions and the different points of zero net proton charge, the shape of the proton charge curve as function of $\mathrm{pH}$ of silica is rather different from that of gibbsite. Also the surface potential behavior is different: the surface potential of silica is highly non-Nernstian (i.e. strongly dependent on the ionic strength and non-linear with a change in $\mathrm{pH}$ ), while the surface potential of gibbsite is approximately Nernstian (linear with a pH change and nearly independent of the ionic strength) [76-78]. Other metal oxide surfaces have also fractional formal charges and react in a similar way as gibbsite. At constant $\mathrm{pH}$ also the surface potential of metal oxide surfaces is about constant and about independent of further solution conditions, therefore metal oxide surfaces are also called 'constant (surface) potential' surfaces. The fact that most surfaces have multiple types of surface oxygen groups that can react with protons makes the situation more complicated, but the principle difference between silica-type and gibbsite-type behavior remains. The proton charge vs. $\mathrm{pH}$ at different ionic strength values, as observed for gibbsite [81], silica [82] and hematite [83] is depicted in Fig. 5.

The multiple types of surface oxygen groups on silica and metal oxides and a detailed electrical double layer model are of most importance for a good understanding of ion binding other than protons, because these ions often do not react with all surface groups and/or may lose part of their hydration shell depending on the type of surface group (inner-sphere versus outer-sphere complex formation). Information on the type and structure of the adsorption complexes has to be derived from spectroscopic studies and can be used in the extended MUSIC model for ion binding, the CD-MUSIC model [84, 85], where CD stands for charge distribution of the bound ions. For goethite the available parameters allow calculations with many different ions and reliable predictions can be made based on 'model goethite' even in complex situations.

Concluding, silica and metal oxides have rather different surface properties: (i) silica contains both hydrophilic and hydrophobic sites (siloxanes), while the normal metal oxides 
are hydrophilic, (ii) silica has an asymptotic point of zero net proton charge and a highly non-Nernstian surface potential, while the metal oxides charging curves are linear in the point of zero net proton charge and their surface potentials are close to Nernstian. Metal oxide surfaces can be called, to a good approximation, 'constant potential' surfaces. For ion adsorption to goethite accurate parameters are available for many ions so that reliable predictions can be made for soils dominated by goethite.

\subsection{Silicate clays (alumino-silicates)}

A very important fraction of soils are the clays or alumino-silicates. Clay particles are crystalline and found around the world but they are more widespread in temperate areas. A main difference with most metal oxides is that silicate clays have a layered structure [14, 86]. The basic structure is composed of a sheet of tetrahedrons of silicon atoms surrounded by oxygen atoms and a sheet of octahedrons in which an aluminum ion is surrounded by six hydroxyl groups or oxygen atoms. The tetrahedrons in a sheet are linked by three of the oxygens in the tetrahedron with adjacent tetrahedra and arranged in hexagonal rings, which allow the sheet to extend indefinitely in the plane direction; the remaining oxygen is linked to $\mathrm{Al}$ in the octahedral sheet. Also apical oxygen atoms are common to adjoining tetrahedral and octahedral sheets. Combinations of stacked tetrahedral and octahedral sheets are termed layers. Many layers are found in each crystal and in some clays the layers are separated by interlayers in which water and adsorbed cations are found. In nature, cations having nearly the same radius as $\mathrm{Si}^{4+}$ (e.g. $\mathrm{Al}^{3+}$ ) can replace $\mathrm{Si}^{4+}$ in the tetrahedral sheet by isomorphous substitution. Isomorphous substitution can also take place in the octahedral sheet with $\mathrm{Al}^{3+}$ being replaced by a similar-sized cation (e.g. $\mathrm{Mg}^{2+}$ ). The substitution finds mostly place with cations that have a lower valence than either $\mathrm{Si}^{4+}$ or $\mathrm{Al}^{3+}$ and this results in unsatisfied negative charges within the crystal, therefore isomorphous substitution is the primary source 
411 of the negative charge of clay surfaces. The negative charges attract cations from the soil 412 solution.

413 Based on the number and arrangement of tetrahedral and octahedral sheets contained in 414 the crystal units or layers, silicate clays are classified into two different groups: i) 1:1 type 415 minerals in which the layers have a TO structure build up of one tetrahedral (T) sheet and 416 one octahedral (O) sheet and ii) 2:1 type minerals with a TOT layer structure. The stacking 417 of layers in a 1:1 type crystal leads to two different basal plates: a T-layer with surface 418 oxygens and an O-layer with surface hydroxyls. For a 2:1 type mineral both basal plates are 419 T-layers with surface oxygens. The surface oxygens of the tetrahedra in a T-surface layer 420 form a siloxane surface; in the centers of the 6-membered rings of the tetrahedra a cavity is 421 formed of about $0.23 \mathrm{~nm}$ that acts as a specific site for cations if their size matches the size 422 of the cavity [87]. Although the siloxane sites make the surface hydrophobic, the charged 423 sites that occur due to the isomorphic substitution and the presence of hydrated cations 424 make the surface considerably less hydrophobic than a neutral siloxane surface. The basal 425 surface of the T-layer is very common, it occurs for all clays; as different clays have 426 different degrees of isomorphous substitution the hydrophobicity-hydrophilicity of the 427 T-surface also varies, the higher the structural charge density, the lower the hydrophobicity. 428 The surface oxygens of the O-layer are similar to the double coordinated oxygens of the 429 planar 001 face of gibbsite and form surface hydroxyls that are fully charge satisfied and 430 unreactive with respect to protons. The structural surface charge and the presence of the 431 hydroxyls make the O-basal plate hydrophilic.

432 The edges of the clay particles have unsatisfied bonds that are quite reactive with respect 433 to protons. For the T-layer edge these are the silanol groups that can protonate: $\equiv \mathrm{SiO}^{-}+\mathrm{H}^{+}$ $434=\equiv \mathrm{SiOH}^{0}$. For the O-layers the edge face has doubly and singly coordinated oxygen groups. 435 The doubly coordinated $\equiv \mathrm{Al}_{2}-\mathrm{OH}^{0}$ groups are inert in the normal $\mathrm{pH}$ range, the singly 436 coordinated $\equiv \mathrm{AlOH}^{1 / 2-}$ groups at the O-edges are the reactive groups; for their protonation 
437 one can thus write $\equiv \mathrm{AlOH}^{1 / 2-}+\mathrm{H}^{+}=\equiv \mathrm{AlOH}_{2}{ }^{1 / 2+}$. Note that the site density of $\equiv \mathrm{SiO}^{-}$at the 438 edges for a 2:1 clay will be higher than that at the edges of a 1:1 clay. In the normal $\mathrm{pH}$ 439 range the silanol sites will be partly negative, partly neutral, while the aluminol sites will be 440 partly positive, partly negative, the edge surface is therefore amphoteric. The above is the 441 simplest representation of the edge, in practice the situation is more complicated because of 442 non-ideal structures and end effects that cause bond-length relaxation and charge 443 redistribution at the edge surface [88-90].

444 Proton binding to clays is considerably more complex than to silica or metal oxides; 445 protons will participate in the cation exchange at the basal planes and in the protonation or 446 deprotonation of the edges. Because of the presence of the structural charge on the basal 447 planes next to the variable charge of the edge, one deals with a patchwise heterogeneous 448 surface and for such surfaces the points of zero net proton charge are dependent on the 449 differences between the patches and the electrolyte concentration [83, 91]. One of the 450 complications is that the proton binding to the edges can be affected by the structural charge 451 due to 'spillover' of the electrostatic potential between basal and edge surface. In a 452 relatively simple model Avena et al. [92] incorporated both the structural charges that can 453 exhibit ion exchange and the variable edge charge that can change with $\mathrm{pH}$ according to a 454 simple proton association - dissociation reaction. The spillover of the potential is taken into 455 account by smearing-out the total particle charge over the entire surface. This model 456 captures the basic features of the proton charging and exchange reasonably well, but the 457 parameters have to be fitted. Fig. 6 depicts the measured proton charge curves for montmorillonite that clearly show the shifts of the points of zero net proton charge, together 459 with the calculated curves [92].

460 When the spillover effect is calculated in a sophisticated way and the site structure and reactivity are modeled with the MUSIC approach with predicted $\mathrm{pK}_{\mathrm{H}}$ values the situation is considerably more complicated. Bourg et al. [88] mention the various models that have been 
used to describe the proton charging of montmorillonite and discuss the experimental results and the most promising sophisticated models.

It is also instructive to briefly discuss how the layers are held together for the main clay types. In the 1:1 type crystals, with kaolinite as most prominent example, the layers are held together by hydrogen bonds; as these bonds are strong, the layers are closely held together in stacks that are large in size, have no internal surfaces and do not swell when wetted. Also the isomorphous substitution is low, consequently, these minerals have a relatively low adsorption capacity for cations. The so-called cation exchange capacity or CEC is largely due to cation adsorption on the edges of the particles and therefore relatively strongly depending on the $\mathrm{pH}$. The 2:1 clays can be subdivided in four general groups depending on how strongly the layers are bound together: micas, chlorites, vermiculites and smectites. In micas a relatively high fraction ( $25 \%)$ of the silicon atoms in the tetrahedral sheets have been replaced by aluminum. The resulting large negative charge is satisfied by potassium ions held rigidly between the adjoining 2:1 layers and preventing expansion of the crystal. As a consequence of the strong binding they have a fairly large stacking of layers and are non-expanding. They resemble the 1:1 type clays somewhat and have a relatively low CEC that is also fairly $\mathrm{pH}$ dependent. The chlorites are also non-expansive, the adjacent negative layers are held together by a positively charged magnesium-dominated octahedral sheet in the interlayer between the two 2:1 layers. Chlorites have particle sizes, CECs, and physical properties similar to those of fine-grained micas. Vermiculites have a significant substitution of aluminum for silicon in the tetrahedral sheets as well as some substitution of magnesium for aluminum in the octahedral sheet, but lower than in micas. The individual 2:1 layers are held together only loosely by $\mathrm{Mg}^{2+}$ and $\mathrm{Ca}^{2+}$ ions that act as bridges between the structural charges in the adjoining layers. Water molecules are attracted between the layers and vermiculites expand when wet and shrink when dry. Due to the weak attraction between the layers the surfaces adjoining the interlayer are also available for adsorption and this leads to 
a very large total interface and a large CEC. Due to the limited expansion and the high CEC, vermiculites have a high affinity for weakly hydrated cations such as $\mathrm{K}^{+}, \mathrm{NH}^{4+}$ and $\mathrm{Cs}^{+}$. The final group contains the smectites, with montmorillonite as well known member. In the smectites some magnesium has been substituted for aluminum in the octahedral sheet and some aluminum for silicon in the tetrahedral sheet, but the degree of substitution is less than in vermiculite and the interlayer is larger. Due to the relatively low structural negative charge, $\mathrm{K}^{+}$fixation is lower than in vermiculite. As in vermiculite, the cations in the interlayer are exchangeable and this gives rise to high CECs. It may be clear that also surfactant binding to non-expanding clays is far simpler than that to the expanding clays.

\subsection{Concluding remarks}

Comparing the ion binding models for metal oxides and silica with those of humic substances it follows that the electrostatic part of the model for metal oxides and silica is far more complicated than that of the humic substances, especially when the adsorption of ions other than protons is considered; for humic substances the heterogeneity is most important. Adequate understanding of proton adsorption to silicate clays is only possible when a distinction is made between the edges and the basal planes and considering the spillover of the electrostatic potential of the basal planes to the edges. For all systems the proton charging may change when another species is adsorbed (primary charge adjustment) due to generic electrostatic effects and/or site competition. The different particles also differ in hydrophobicity. Humic substances have many hydrophobic parts, the silica surface is partly hydrophobic and the metal oxides are hydrophilic. Furthermore, adsorption on silica should be distinguished from that on the metal oxides, because of the differences in proton charging behavior and hydrophobicity. With silicate clays the edges are hydrophilic and the basal planes of 2:1 clays are hydrophobic, but the hydrophobicity is weakened by the presence of the structural charge and the adsorbed cations. For kaolinite (1:1 clay) the basal 
515 siloxane surface is fairly hydrophobic, but the basal aluminol surface is hydrophilic; with

516 montmorillonite (2:1 clay) the two basal siloxane surfaces are less hydrophobic than the

517 siloxane surface of kaolinite due to the greater degree of isomorphic substitution.

518

\section{Surfactant binding to humic substances}

\subsection{Early experimental studies and isotherms measured with surfactant electrodes}

The investigations of surfactant binding to humic and fulvic acids are relatively recent, still limited, and have not been reviewed. Therefore, the most important binding studies will be discussed. Early reports are of Tombacz [93, 94] who investigated alkylammonium -

525 humate complexes with X-ray and interfacial tension measurements. Binding isotherms to dissolved humic substances were reported for the first time in 1996 by Traina et al. [95], who used an ultra-centrifugation technique and fluorescence quenching to obtain the binding characteristics of C10-, C12-, and C14-linear alkyl-benzene sulfonates (LAS; in 0.03M NaCl and 0.01M CaCl 2 ) to dissolved humic substances. By using pulse-field gradient NMR Otto et al. [96] found that humic substances enhance the aggregation of the anionic sodium dodecylsulfate (SDS) prior to micellization and that they form ion pairs with cationic C16-trimethylammonium bromide.

The first isotherms ranging from low surfactant concentrations till the CMC were reported by Koopal et al. [97], who used a potentiometric method with a surfactant 535 electrode to measure the equilibrium surfactant concentration. Three surfactants were used, 536 one anionic (SDS) and two cationic (C12- and C16-pyridinium chloride or C12PC and C16PC) and the binding to purified Aldrich humic acid (PAHA) was investigated at three $\mathrm{pH}$ values $(5,7,10)$ at $0.025 \mathrm{M}$ background electrolyte concentration. No binding could be observed for SDS under the given conditions. The isotherms of the cationic surfactants were independent of the humic acid (HA) content in solution (0.2-0.5 g/L), which indicates that 
541 the results were not affected by possible dynamic aggregation/segregation of $\mathrm{HA}$. The

542 observed isotherms for the cationic surfactants are depicted in Fig. 7; the binding is plotted 543 vs. $\mathrm{c} / \mathrm{CMC}^{0}$, where $\mathrm{c}$ is the surfactant concentration and $\mathrm{CMC}^{0}$ is the $\mathrm{CMC}$ in the absence of

544 HA. The binding of C12PC and C16PC increased with increasing $\mathrm{pH}$ to maxima that 545 corresponded to the negative charge of the HA (arrows in Fig. 7). By plotting the binding vs. $546 \log \mathrm{c} / \mathrm{CMC}^{0}$ the isotherms of $\mathrm{C} 12 \mathrm{PC}$ and $\mathrm{C} 16 \mathrm{PC}$ merged largely, indicating that next to the 547 electrostatic attraction hydrophobic bonding is important. When the charge neutralization 548 point was reached the cationic surfactant-PAHA complexes precipitated, which occurred at 549 approximately $10 \%$ of the CMC. For C16PC, the precipitation was complete, but in the case 550 of C12PC, a noticeable fraction of PAHA remained in solution.

551 Subsequent binding studies were made by Yee et al. [98-101], Matsuada et al. [102] and 552 Ishiguro et al. [103], who all used the potentiometric technique with a surfactant electrode 553 to measure the equilibrium surfactant concentrations. Yee et al. [100] also investigated the 554 binding of SDS to HA using Aso HA (AHA) derived from the Aso area of Kyushu Island, 555 Japan. At pH 9.18 and low ionic strength (0.03M) no binding could be observed between 556 SDS and AHA with either the potentiometric or dynamic light scattering (DLS) method, 557 which confirmed the result of Koopal et al. [97]. However, with the DLS method some 558 interaction could be detected at $\mathrm{pH} 3.98$ and high ionic strength $(0.10 \mathrm{M})$. The attraction can 559 be explained by the fact that at $0.1 \mathrm{M}$ the electrostatic repulsion is suppressed, but the 560 hydrophobic attraction remains the same and the net effect is a weak attraction. Temperature 561 studies of C12-pyridiniumbromide binding to Aso fulvic (AFA) and AHA at pH 9.18 and 562 ionic strength of $0.03 \mathrm{M}$ showed that the binding of $\mathrm{C} 12 \mathrm{P}^{+}$with AFA was endothermic, i.e. 563 driven by a positive entropy [99], while the enthalpy of $\mathrm{C}_{12} \mathrm{P}^{+}$binding with AHA was 564 slightly negative [101]. For AFA different binding modes were observed at two pH regions, 565 i.e., cooperative binding at $\mathrm{pH}>7$ and non-cooperative binding at $\mathrm{pH}<7$, see Fig. 8 [99]. 
For the AHA-C12P ${ }^{+}$system no cooperative binding was observed, but the $\mathrm{C}_{12} \mathrm{P}^{+}$ concentration at which the first binding was observed was much lower for AHA than for 568 AFA [101]. The latter reflects that AHA is more hydrophobic than AFA because the acidic type of groups to which the surfactant head groups bind are very similar for FA and HA and the negative charge density of FA is in general larger than that of HA. The $\mathrm{C}_{12} \mathrm{P}^{+}$binding to AFA did rise steeply just before the CMC, i.e. AFA-C12P ${ }^{+}$cooperation occurred very close to the CMC. The isotherm of $\mathrm{C} 12 \mathrm{P}^{+}$binding to AHA leveled-off just before the CMC as the binding was close to its maximum value. The behavior of the AFA-C12P ${ }^{+}$system at $\mathrm{pH}>7$ could therefore also be caused by enhanced $\mathrm{C} 12 \mathrm{P}^{+}$micellization in the presence of AFA, 575 instead of typical $\mathrm{C}_{2} \mathrm{P}^{+}$binding to AFA. The stronger hydrophobicity of AHA than of AFA 576 was confirmed by studying the effect of the aliphatic chain length $(n)$ of the surfactant tail 577 ( $n: 12,14,16)$ [98]; the results are plotted in Fig. 9. In all cases the comparable isotherms started for AHA at concentrations that were $10 \mathrm{x}$ lower than for AFA. The isotherms shifted with increasing chain length roughly with the same factor as the CMC, but the shift was larger for AFA than for AHA. The results for AHA correspond with the observations of Koopal et al. [97] and Ishiguro et al. [103].

It should be realized that two different hydrophobic interactions have to be considered with surfactant-HS interaction: (i) the hydrophobic effect of transferring the hydrocarbon tail of surfactant into the hydrophobic parts of humic substances (HS) and (ii) the lateral 585 hydrophobic attraction between bound surfactants; (i) contributes to the greater binding 586 strength of HA than FA and (ii) may cause cooperative binding. However, when the 587 hydrophobic effect of transfer of the tail into the hydrophobic parts of HS is large, than the extra effect of (ii) will be small. This explains why (ii) is larger for AFA than for AHA.

Yee et al. [99, 101] also investigated the role of the HS content $(0.2-0.5 \mathrm{~g} / \mathrm{L}), \mathrm{pH}$ and ionic strength. In accordance with Koopal et al. [97], no HS concentration dependence of 591 binding was observed in both systems; possible self-aggregation of HS did not affect the 
592 binding. The binding of $\mathrm{C}_{12} \mathrm{P}^{+}$increased with increasing $\mathrm{pH}$, similarly as observed by 593 Koopal et al. [97] for $\mathrm{C}_{12} \mathrm{P}^{+}$and $\mathrm{C} 16 \mathrm{P}^{+}$, and this can be explained by the increase of the 594 negative charge of HS with increasing $\mathrm{pH}$. The bound amount decreased with increasing 595 ionic strength, due to the screening of the electrostatic attraction between surfactant ions 596 and HS; the effect was larger for AHA than for AFA. With DLS the hydrodynamic radii of 597 the AHA-C12PB (PB = pyridinium bromide) and AFA-C12PB complexes were measured at $598 \mathrm{pH} 9.2$ at ionic strength values of $0.03 \mathrm{M}$ and $0.1 \mathrm{M}$ for a constant concentration of AFA or 599 AHA of $0.05 \mathrm{~g} / \mathrm{L}$. In the absence of surfactant no reliable average size could be measured, 600 but in the presence of surfactant good results were obtained. At low surfactant binding the 601 aggregate diameters were about $200 \mathrm{~nm}$ and with increasing surfactant binding up to 4

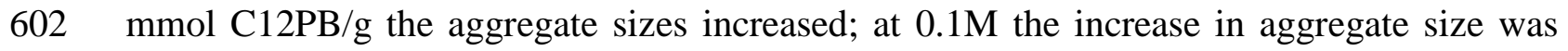
603 similar for AFA and AHA $(\sim 1400 \mathrm{~nm})$, while at 0.03M the increase in size was somewhat 604 lower for AFA ( 1200 nm) but considerably less for AHA ( 500 nm). The difference might 605 be due to the fact that the particle concentration in the case of AFA is much higher; at high 606 ionic strength rapid aggregation may occur for both HS, while at $0.03 \mathrm{M}$ the kinetics are 607 important.

608 The binding of Cn-trimethylammonium $\left(\mathrm{CnTA}^{+}\right)$ions to AFA and AHA has been studied 609 by Yee et al. [100] and Matsuda et al. [102]. Yee et al. compared the binding of C12TA ${ }^{+}$ 610 with that of $\mathrm{C} 12 \mathrm{P}^{+}$at $\mathrm{pH} 9.2$ and $0.03 \mathrm{M}$ at $25{ }^{\circ} \mathrm{C}$. The binding of $\mathrm{C}^{2} \mathrm{TA}^{+}$to AFA or AHA 611 was weaker than that of $\mathrm{C}_{12} \mathrm{P}^{+}$, presumably due to the relatively large size of the headgroup

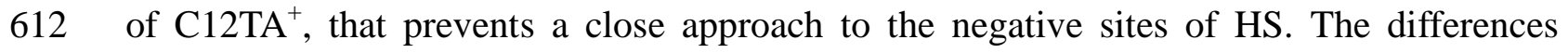
613 between the $\mathrm{CnTA}^{+}$isotherms for AFA and AHA were similar as in the case of

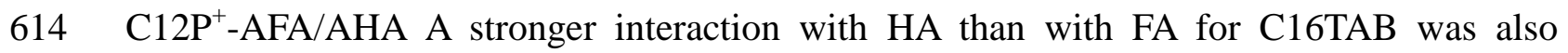
615 observed by Otto et al. [96] using NMR diffusion analysis. The surfactant head group 616 structure also affects the aggregation behavior: the hydrodynamic diameters of 617 AFA-C12TAB and AHA-C12TAB aggregates were smaller than those of AFA-C12PB and 
618 AHA-C12PB aggregates. Similarly as for C12PB the aggregate size of the HS-C12TAB 619 aggregates increased with surfactant binding and the AFA-C12TAB aggregates were larger 620 than those of AHA-C12TAB. Thieme and Niemeyer [104] also observed an increase in size 621 of HS-C12TAB aggregates with increasing C12TAB binding.

Matsuda et al. [102] investigated first the binding of $\mathrm{C}_{12 \mathrm{TA}^{+}}$and $\mathrm{C}_{10 \mathrm{TA}}{ }^{+}$surfactants to PHA (derived from an HA-rich layer in Heilongjiang, China) at $\mathrm{pH} 8$ and different $\mathrm{NaCl}$ concentrations (0-50 mM) and subsequently that in the presence of divalent metal ions $(\mathrm{Cu}$, $\mathrm{Cd}, \mathrm{Zn}, \mathrm{Pb}$, and $\mathrm{Ca})$ at $\mathrm{pH}$ 8. With respect to $\mathrm{C}_{16 \mathrm{TA}^{+}}$the binding curves of $\mathrm{C} 12 \mathrm{TA}^{+}$were shifted to higher surfactant concentrations; the shift was comparable to the shift in CMC. As 627 stated before, the stronger binding for C12TAB than C10TAB indicates the role of the 628 hydrophobic interaction between the tail of the surfactant ions and the hydrophobic parts of 629 PHA. The affinity of $\mathrm{C}_{2} \mathrm{TA}^{+}$for PHA decreased linearly with the square root of the $\mathrm{NaCl}$ concentration. In the presence of $0.5 \mathrm{mM}$ divalent metal salts, the PHA-CnTA ${ }^{+}$binding decreased significantly, which can be explained by site competition and screening of the electrostatic attraction. The effect of the different metal ions on C12TAB binding to PHA was similar, but for C10TAB binding to PHA the effect decreases in the order $\mathrm{Cd}, \mathrm{Zn}, \mathrm{Pb}$, $634 \mathrm{Ca}$, and $\mathrm{Cu}$.

Ishiguro et al. [103] studied the binding of Cn-pyridinium chloride (C12PC and C16PC) to purified Aldrich humic acid (PAHA), Dando humic acid (DHA, Japan), Inogashira humic 637 acid (IHA, Japan), Laurentian fulvic acid (LFA, Canada) and Strichen Bs fulvic acid (SFA, 638 Netherlands) at pH 5 and $0.005 \mathrm{M} \mathrm{NaCl}$, and C16PC binding to PAHA was also studied at $6390.1 \mathrm{M} \mathrm{NaCl}$. Besides surfactant isotherms also titrations of HS with surfactant were carried 640 out using an automatic titrator combined with a Mütek particle charge detector (M-PCD) $641[105,106]$. This M-PCD-method is well suited to measure the charge sign of colloidal 642 particles and in the case of titrations with a complexing agent of opposite charge the 643 iso-electric point (IEP) can be detected. The M-PCD titrations were used to determine the 
644 charge of the $\mathrm{HS}$ samples at the given conditions (pH 5 and $0.005 \mathrm{M} \mathrm{NaCl}$ ) with

645 poly-DADMAC (poly-diallyldimethylammonium chloride) a strong cationic polyelectrolyte

$646[107,108]$ and with $\mathrm{C} 16 \mathrm{P}^{+}$. For this charge determination the IEP is required. For the HA

647 samples the charges determined with $\mathrm{C}_{16 \mathrm{P}^{+}}$were slightly higher than those observed with

648 poly-DADMAC, this could be explained by better screening and stronger charge adaptation

649 in the case of $\mathrm{C}_{16 \mathrm{P}^{+}}$. For the two FA samples the charges obtained with $\mathrm{C} 16 \mathrm{P}^{+}$were

650 significantly larger than those measured with poly-DADMAC. This difference was

651 explained by the relatively low hydrophobicity and corresponding relatively low affinity

652 between FA and $\mathrm{C}^{2} \mathrm{P}^{+}$. By binding of $\mathrm{C} 16 \mathrm{P}^{+}$a surfactant-FA complex becomes more

653 hydrophobic, this enhances the affinity and the isotherm becomes very steep; for such

654 conditions the rate of titration was likely too fast and leading to too high IEPs. With C12P ${ }^{+}$

655 the M-PCD method was only used to obtain at the given conditions the bound amount of

656 surfactant at the IEP and the corresponding solution concentration of surfactant.

657 Comparison of the $\mathrm{C}_{16 \mathrm{P}^{+}}$results with those of $\mathrm{C}_{12 \mathrm{P}^{+}}$showed that for PAHA the bound

658 amounts (mol/g) of $\mathrm{C}_{16 \mathrm{P}^{+}}$and $\mathrm{C}^{2} \mathrm{P}^{+}$were the same, i.e. at the IEP the charge associated

659 with the HS was neutralized by bound surfactant ions. For the other HS samples the bound

660 amount of $\mathrm{C}_{12} \mathrm{P}^{+} / \mathrm{C} 12 \mathrm{PC}$ at the IEP was larger than that of $\mathrm{C} 16 \mathrm{P}^{+}$. This must indicate that at

661 the IEP some C12PC was included in the complex, as there is no reason to believe that the

662 screening of the HS charge by $\mathrm{C}_{12} \mathrm{P}^{+}$is better than that by $\mathrm{C}_{16} \mathrm{P}^{+}$when the HS-C12 $\mathrm{P}^{+}$

663 affinity is weaker. Chloride inclusion is most likely caused by the fact that around the IEP

664 the C12PC concentration in solution is relatively high. The behavior of $\mathrm{CnP}^{+}$surfactants

665 often shows a dependence on the type of counterion, indicating incomplete dissociation

666 [109]. With C16PC the affinity for HS is so high that the amount of C16PC left in solution

667 is very low. Therefore, binding of uncharged C16PC to HS is insignificant for C16PC. The

668 M-PCD titrations with the surfactants also reveal that the surfactant-HS complexes reached

669 their IEP before the CMC was reached. Around the IEP some flocculation of the complexes 
670 could be observed, and upon continuation of the titration charge reversal of the aggregated

671 complexes occurred. This may have lead to partial re-dispersion of the aggregates. Shang 672 and Rice [110] and Subbiah and Mishra [111] investigated HA-C16TB complexes by, 673 respectively, small-angle X-ray scattering and synchronous and excitation emission matrix 674 fluorescence, both found (partially) reversible flocculation around the CMC. Shang and 675 Rice used a purified peat HA; Subbiah and Mishra, who used Aldrich HA that was purified 676 by two filtration steps, also investigated SDS and Triton-X100 (t-Octyl- $\mathrm{C}_{6} \mathrm{H}_{4}-\left(\mathrm{OCH}_{2} \mathrm{CH}_{2}\right)_{\mathrm{x}} \mathrm{OH}$, $677 x=9-10$ ), a nonionic surfactant, and found only very weak interactions for these surfactants. 678 Binding isotherms of $\mathrm{C} 16 \mathrm{P}^{+}$to $\mathrm{PAHA}$ at two ionic strength values reported in [103] are 679 reproduced in Fig. 10. The binding is plotted in panel a) vs. $\log \mathrm{C}_{\mathrm{eq}}$ (surfactant) and in panel 680 b) the same result is depicted but now as double logarithmic plot. The horizontal dashed line 681 indicates the bound amount of $\mathrm{C}_{16} \mathrm{P}^{+}$and the approximate concentration at the IEP as 682 measured with the M-PCD. The thin vertical lines in the top of the figures indicate the CMC 683 values. In panel a) also the proton release due to $\mathrm{C}_{16} \mathrm{P}^{+}$binding is included. Two striking effects can be observed: (i) the isotherms at different salt concentration intersect at the IEP of the complex and (ii) close to or at the CMC the isotherms rise steeply. The latter is an 686 artifact; it is binding of surfactant in micelles, not to PAHA. At the common intersection 687 point $(\mathrm{CIP}=\mathrm{IEP})$ the charge of PAHA is neutralized by $\mathrm{C}_{16 \mathrm{P}^{+}}$. The CIP occurs at a 688 surfactant concentration smaller than the $\mathrm{CMC}$ and the $\mathrm{C} 16 \mathrm{P}^{+}$binding continues till the 689 CMC because of hydrophobic attraction. Binding above the CIP=IEP indicates that $\mathrm{C} 16 \mathrm{P}^{+}$ 690 binds super-equivalently to PAHA. Before the CIP, where the complex is negative, the salt concentration screens the negative charge of the complex and this diminishes the head group surface attraction, at the CIP the complex is net uncharged and salt has no effect, beyond the CIP the complex is positive and the salt concentration screens the repulsion 694 between the head groups. As a consequence the salt concentration dependence inverses at 695 the CIP=IEP. The proton release is up to the CIP very small, $\mathrm{C}_{16 \mathrm{P}^{+}}$binding occurs on 
696 already dissociated groups; beyond the CIP the proton release increases and is only slightly smaller than the increase in surfactant binding, so most of the increment in surfactant charge 698 is balanced by proton release.

699 For sake of comparison the binding of $\mathrm{C}_{12} \mathrm{P}^{+}$to sodium polystyrene sulfonate (SPSS), a 700 flexible linear anionic polyelectrolyte, is also plotted in Fig. 10, panels c) and d) [112]. The $701{\mathrm{C} 12 \mathrm{P}^{+}}^{+}$isotherms of SPSS at the three different ionic strength values merge before the CMC 702 (the measurements are not accurate enough to observe a CIP); however, for SPSS-C16 ${ }^{+}$a 703 clear CIP could be detected [112]. The double logarithmic plots (b and d) are best suited to 704 investigate cooperativity of binding. It should be remarked that sometimes the S-shape of 705 single logarithmic plots is used to indicate cooperativity, but this leads easily to erroneous 706 results, because the Langmuir isotherm (no cooperativity) also has an S-shape in these plots. 707 Firstly, the binding of $\mathrm{C} 12 \mathrm{P}^{+}$to SPSS is considered as this system shows the various aspects 708 most clearly (this is the reason that in Fig. 10 SPSS-C12P $^{+}$was preferred above 709 SPSS-C16 ${ }^{+}$). Along the isotherm four regions can be distinguished. Region I, at the very 710 low surfactant binding (or surfactant concentration) is the region where the slope of the 711 isotherm is unity, this is the "Henry region" where binding and concentration are 712 proportional; the surfactant is ideally bound on isolated sites of equal energy. For 713 SPSS-C16P ${ }^{+}$, region I cannot be observed; the slope is very steep from the start due to the 714 strong cooperativity [112]. In region II, the slope of isotherms becomes larger than unity, 715 which clearly indicates cooperativity. The transition point of region I and II is defined as the 716 critical association concentration (CAC); at the CAC aggregation of surfactant starts. In region II the number of aggregates along the polyelectrolyte chain increases with increasing 718 surfactant concentration. In region III the slope of the isotherms gradually lowers and 719 becomes smaller than unity due to three factors: 1) the attractive electrostatic interaction 720 becomes smaller due to the decrease of the negative charge of SPSS-C12P ${ }^{+}$complex, 2) the 721 mixing entropy of "free" and $\mathrm{C}^{2} \mathrm{P}^{+}$-occupied sites decreases and 3) polymer chain 
conformations become more restricted. The last factor will be of little importance for HS, which is internally structured. The CIP marks the end of region III and the start of region IV, the negative charge of the polymer is compensated by the positive charge of bound surfactant. In region IV the aggregates grow slightly further and the SPSS-C12 ${ }^{+}$complex becomes increasingly positive.

Let's now return to the PAHA-C16 ${ }^{+}$system [103]; in the double logarithmic plot the 'Henry slope' is indicated by the dashed line. At $0.005 \mathrm{M} \mathrm{NaCl}$ a CAC and cooperativity cannot be observed, but there is a slight kink in the isotherm that could be the transition point between regions I and II. Region III (up to the CIP) and region IV beyond the CIP can be clearly observed. At $0.1 \mathrm{M}$ the isotherm starts with a slope higher than unity, that implies some cooperativity is observed at low surfactant concentration, but the CAC cannot be established. The increase in cooperativity with increasing electrolyte concentration occurs because the electrostatic repulsion between the bound surfactants decreases.

In Fig. 11 the binding at $0.005 \mathrm{M} \mathrm{NaCl}$ of $\mathrm{C}_{16} \mathrm{P}^{+}$is compared to that of $\mathrm{C}^{2} \mathrm{P}^{+}$. The influence of carbon chain length on binding can be clearly observed. The $\mathrm{C}_{16} \mathrm{P}^{+}$binds at much lower surfactant concentrations than $\mathrm{C}_{12} \mathrm{P}^{+}$and the isotherm is somewhat steeper. The larger affinity for $\mathrm{C}_{16} \mathrm{P}^{+}$is largely due to the fact that $\mathrm{C}_{16} \mathrm{P}^{+}$'hates' the aqueous solution more than $\mathrm{C}_{12} \mathrm{P}^{+}$, because the shift of the two isotherms is similar to that of the CMC. This reveals the importance of the hydrophobic effect for the binding.

The $\mathrm{C}_{12} \mathrm{P}^{+}$binding isotherms for the different humic and fulvic acids at $0.005 \mathrm{M} \mathrm{NaCl}$ are compared in Fig. 12. The shape of the binding isotherms to the humic acids is distinctly different from that of the fulvic acids and the $\mathrm{C}_{12} \mathrm{P}^{+}$binding decreases in the order of PAHA $>>$ IHA $\approx$ DHA $>>$ SFA $\approx$ LFA. The order ranks the HS with respect to hydrophobicity, PAHA is most hydrophobic the FA's the least. Cooperativity is not observed at pH 5 and $0.005 \mathrm{M} \mathrm{NaCl}$ because the slopes of the curves are almost unity or smaller than unity. These results correspond with the findings of Yee et al. [98, 100, 101] discussed above. 


\subsection{Surfactant binding measured by solid-phase micro-extraction}

Recently, Chen et al. [113] have demonstrated that polyacrylate (PA) coated microfiber sorbs organic cations via ion exchange at the carboxylic groups. When the binding isotherm is known, it can be used to determine the freely dissolved concentration of the given cationic surfactants. The free surfactant concentration in a HS-surfactant system can be obtained by extracting the surfactant bound to PA and determine the extracted amount by HPLC, hereafter the calibration line gives the equilibrium surfactant concentration. The method is called solid-phase micro-extraction (SPME). The method will work well when the surfactant binding to PA is negligible compared to the binding to HS. The double logarithmic representation of the surfactant isotherm to PA fiber is used as calibration curve for the dissolved surfactant, because in this way the isotherm is linear in the low concentration range. Binding isotherms of hexadecyl-trimethylammonium chloride (C16TAC) and benzyldimethyl-dodecyl-ammonium chloride (C12BDAC) to PA are reported as examples; the isotherms of these two surfactants coincide, while their CMCs differ by more than a factor 10. Based on the difference in hydrophobicity this must imply that PA is strongly hydrophilic and that there is-no hydrophobic contribution to the specific affinity for the PA-carboxylic groups, mainly the surfactant head group chemistry seems important. The linear part of the log-log isotherms of the two surfactants cover a concentration range from about $10^{-10}$ to $10^{-6.5} \mathrm{M}$. The upper limit of this range is $10^{3}$ to $10^{4}$ times smaller than the CMCs. This implies that accurate measurements are only possible in an equilibrium surfactant concentration range considerably below the CMC, for higher concentrations the sensitivity lowers with increasing concentration. Therefore, the method is most valuable for the linear part of the log-log surfactant HS isotherms. The preliminary conclusion is that the PA-SPME will be of substantial added value in the low surfactant concentration range, but that the surfactant electrode will be superior in the higher 
774 concentration range $\left(>10^{-3} \mathrm{CMC}\right)$. Anyhow, this new technique is highly welcome and will

775 promote further research of cationic surfactant binding. As example a series of binding

776 isotherms of C12BDAC to purified Aldrich humic acid (purified AHA) at pH 6 and

777 different salts and salt concentrations were measured. Chen et al. [114] continued the latter

778 study in a subsequent paper in which the $\mathrm{pH}$ range of the measurements was extended to $\mathrm{pH}$

779 3, some results are reproduced in Fig. 13.

\subsection{Modeling surfactant - humic substance interaction}

Ishiguro and Koopal [115] modeled the results of C12PC and C16PC to different humic and fulvic acids with the NICA-Donnan model [38, 43, 54-56] (see also section 7) under the assumption that the surfactant $(s)$ was dominant: $\left(k_{s} c_{s}\right)^{n s} \approx \Sigma\left(k_{i} c_{i}\right)^{n i}$ with $k_{i}$ the intrinsic affinity, $c_{i}$ the concentration and $n i$ the stoichiometry of component $i$. This implies that only the low affinity or carboxylic groups were considered and that the surfactant binding to these groups was larger than the proton binding. For these conditions the NICA equation simplifies to a Hill type equation, but the cooperativity parameter, $m$, includes the cooperativity $n s$ and the site heterogeneity parameter, $p: m=n s \bullet p$. This Hill-type equation is complemented with the Donnan model that accounts in a simple way for the electrostatic interactions by assuming that for a given set of conditions there is only one electrostatic 792 potential that governs the behavior in the Donnan phase. With the combined model the 793 experimental results for the binding of the different surfactants to the different HAs at 794 different salt concentration and $\mathrm{pH}$ could be well described. For the FAs only the linear part 795 of the log-log isotherms could be described well.

796 Chen et al. [114] also modeled the surfactant isotherms with the NICA-Donnan model. 797 As the measured isotherms were restricted to the linear range, they made the assumption 798 that the total binding was hardly or not affected by the surfactant (s) binding: $\left(k_{s} c_{s}\right)^{n s}<<$ $799 \Sigma\left(k_{i} c_{i}\right)^{n i}$ with $k_{i}$ the intrinsic affinity, $c_{i}$ the concentration and ni the stoichiometry of 
800 component $i$. In this limit the NICA equation becomes a Hill-type equation $(m=n s)$ for 801 which $\left(k_{s} C_{s}\right)^{n s}<<1$, which has the same form as the classical Freundlich equation. By 802 combining this equation with the Donnan model to incorporate the electrostatics and using

803 the generic description of HA as provided by Milne et al. [55, 56] the salt effects at $\mathrm{pH} 6$ 804 could all be explained well.

805 By considering the successful modeling attempts by Ishiguro and Koopal [115] and Chen 806 et al. [114] and the fact that two different limiting forms of the NICA equation both lead to a 807 Hill type equation also explains the success of the use of the Hill equation in the work of 808 Yee et al. [98-101]. However, as the Donnan (electrostatic) part was omitted by Yee et al. no 809 explanation could be provided for $\mathrm{pH}$ and salt effects. For more information on the 810 NICA-Donnan equation section 7 should be consulted.

\subsection{Sorption of organic cations and risk assessment}

Droge and Goss [116] studied the sorption of organic cations to soil organic matter 814 (SOM) using dynamic column experiments (peat mixed with silicium carbide) with 815 different compositions of electrolytes in aqueous eluents. The sorption affinity of the 816 organic cations to Pahokee peat soil (i) strongly decreased with increasing electrolyte 817 concentration, (ii) was higher in $\mathrm{NaCl}$ solutions than in $\mathrm{CaCl}_{2}$ solutions of similar ionic 818 strength, and (iii) was more sensitive to a decrease in $\mathrm{NaCl}$ than to a decrease in $\mathrm{CaCl}_{2}$. 819 Based on the results of this comprehensive study the authors made three remarks. (1) The 820 mass action law equations for ion-exchange reactions predicted trends in a qualitative but 821 not in a quantitative way. (2) Complex models, such as the NICA-Donnan model, are 822 required to fully account for the contributions of ionic interactions to the sorption of organic 823 cations to SOM. (3) The results imply that risk assessment models for organic bases should 824 take ion-exchange processes adequately into account when estimating soil sorption 825 coefficients and bioavailability. These findings indicate that, in general, the NICA-Donnan 
826 model can play an important role in the description of organic cation exchange with soil

827 organic matter.

\section{Surfactant adsorption to metal (hydr)oxides and silicas}

\subsection{General aspects and reviews}

In contrast to the binding of surfactants to HS, the adsorption of surfactants to mineral surfaces has received much attention, because insight in the structure that adsorbed surfactants adopt is vital for practical applications. In older studies most attention was focused on adsorption isotherms and their description. The typical isotherm of ionic

836 surfactants on oppositely charged metal oxides, when depicted as logarithm adsorbed

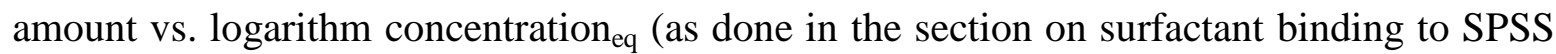
and HS), can be subdivided into four regions. Somasundaran and Fuerstenau [117] have introduced this “four-regions” concept and it has been followed in many studies. Typically, the four-regions isotherm extends over about three decades in concentration and adsorbed amount; two examples of the four-regions isotherm are presented in Fig. 14(a), the isotherm to the left depicts the adsorption of an anionic surfactant (sodium C9-benzene sulfonate) on

843 rutile at low $\mathrm{pH}$, the isotherm at the right shows the adsorption of a cationic surfactant 844 (C12-pyridinium chloride) on rutile at high $\mathrm{pH}$; for sake of comparison the same isotherms 845 are presented in Fig. 14(b) as semi-logarithmic plot that provides better insight in the higher 846 adsorbed amounts close to the CMC [118].

To interpret the four-regions isotherm, the adsorption is best described with the relation $\Gamma=K c^{n}$, where $\Gamma$ is the adsorbed amount, $c$ the concentration divided by the standard state concentration ( $1 \mathrm{~mol} / \mathrm{L}), K$ the effective affinity constant and $n$ the cooperativity. In region I isolated surfactant molecules adsorb and slope-I $=1$ or close to unity, which implies $n_{\mathrm{I}}=1$ 
852 and thus $n_{\text {II }}>1$ which is due to cooperative association of adsorbed molecules caused by 853 hydrophobic attraction. For $n_{\mathrm{II}}>n_{\mathrm{I}}$ the transition $\mathrm{I} / \mathrm{II}$ is called the critical surface

854

855

856

857

858

859

860

861

862

863

864

865

866

867

868

869

870

871

872

873

874

875

876 association concentration (CSAC). At transition II/III the slope of the log-log isotherm mostly decreases, i.e. $n_{\text {III }}<n_{\text {II }}$; for $n_{\text {III }}>1$ there is still cooperative adsorption but the cooperation is less than in region II; for $n_{\mathrm{III}}<1$ there is negative cooperativity likely caused by a decrease in configurational entropy due to high surfactant adsorption. Region IV begins at the CMC, beyond which the chemical potential of the monomeric surfactant remains approximately constant, therefore also the adsorption is constant. In some cases, e.g. for cationic surfactant adsorption on silica at low ionic strength, a four-regions isotherm is observed that does not confirm to the above picture: $n_{\mathrm{II}}<n_{\mathrm{I}}(=1)$ and $n_{\mathrm{II}}<n_{\mathrm{III}}$ with $\mathrm{n}_{\mathrm{III}}>1$, thus region III has the steepest slope. In that case the concentration at which the transition II/III occurs is the CSAC. Although the four-region-type isotherm is well established, especially the interpretation of transition II/III is debated together with the structure of the adsorbed molecules in both adjoining regions. This discussion is enhanced by the results of modern techniques that give information on the adsorbed layer structures. An important contribution to the discussion has been the paper of Manne et al. [119] in which convincing evidence, based on atomic force microscopy (AFM), was presented that surfactants tend to self-assemble at surfaces to form analogues of the micelles in bulk solution. Since then the attention has shifted from adsorption isotherms to modern techniques that provide information on the adsorbed structures. Most reviews of ionic surfactant adsorption pay attention to the four-regions isotherms, but the debate around the four-regions isotherm is still not fully settled and in reviews different views may be expressed. We will return to the four-regions isotherm when the measured isotherms on silica and the metal oxides at constant $\mathrm{pH}$ and different ionic strength values will be discussed. First, the various reviews on surfactant adsorption to silica and metal oxides will be briefly introduced. 
Several authors have reviewed the state of art of surfactant adsorption, mostly with emphasis to their specialism in the field. The older studies are reviewed in $[120,121]$ and Rosen’s book [24] provides a general review of surfactants and surfactant adsorption with emphasis on surfactants and their mixtures both in solution and at interfaces and on the modification of the surface properties by surfactants. After the pioneering work of Manne et al. $[119,122]$ to directly visualize the structure of aggregates formed on surfaces using high resolution scanning probe and atomic force microscopy (AFM), these techniques have been used regularly. This work has been reviewed in [123] and in [119]. Johnson and Nagarajan have presented a theory of surfactant self-assembly on isotropic hydrophobic and hydrophilic surfaces $[124,125]$. The theory is extending the treatment of self-assembly in solution $[28,126]$ with an additional term that accounts for the replacement of the solid surface-water contact by the solid surface-aggregate contact. This contribution depends on the surfactant and surface properties and can be calculated for an assumed geometrical shape of the aggregate; for hydrophobic surfaces and nonionic surfactants it is the Gibbs energy change of replacing surface-water contact by solid surface-aggregate core contact, while for hydrophilic surfaces and ionic surfactants it is (i) the electrostatic interaction between the charged surfactant head groups and the hydrophilic surface, if the surface is charged, plus (ii) the Gibbs energy change associated with displacing water by the surfactant head groups wherever aggregate solid surface contact occurs. The CSAC and the aggregate morphology can be predicted by computing the equilibrium Gibbs energy of formation for a range of assumed geometrical structures and selecting the structure with the lowest Gibbs energy. The CSAC is always predicted to be lower than the bulk phase CMC and a variety of aggregate morphologies are predicted. Morphological transformations of surface aggregates to less energetically favorable structures will occur when the surfactant concentration increases. For hydrophobic surfaces the morphologies include hemispheres, hemicylinders, finite disks, and continuous monolayers depending upon surfactant, solid 
903 surface and surfactant concentration. For hydrophilic surfaces the situation is more complex 904 and in addition to full spheres and cylinders a series of composite structures are proposed 905 which are composed of monolayers oriented with head groups in contact with the surface 906 and covered with either hemispheres, hemicylinders, finite disks, or another monolayer 907 (making the full structure a bilayer). It should be noted that the authors have assumed that 908 monolayer structures with head groups in contact with the surface are instable and 909 transform in composite structures of covered monolayers. This is only correct for surfactant 910 concentrations close to the CMC. Stability and wetting studies have shown that beyond the 911 CSAC the instability and contact angle still increase up to the point where surfactant charge 912 and surface charge compensate each other [127], which points to largely uncovered 913 monolayer type structures or teepee-micelles [128]. Nevertheless, the model provides basic 914 insight in surface aggregation and allows a better judgment of the AMF images that are mostly obtained around or above the CMC.

916 Derived from results obtained with modern measurement techniques Tiberg et al. [129] 917 have discussed in a comprehensive way the adsorption mechanisms and interfacial 918 structures of nonionic and ionic surfactants for both hydrophobic and hydrophilic surfaces. 919 It is an excellent review for a good orientation on surfactant adsorption.

920 Atkin et al. [128] have given a follow-up on this review with emphasis on the adsorption 921 process for ionic surfactants. Traditional equilibrium data (including the four-regions 922 isotherms), discrete aggregation on the substrate (mainly silica), and the morphology of the 923 aggregates are thoroughly discussed. As the AFM is most useful at detecting periodicity, 924 surfactant-substrate combinations that produce highly regular morphologies produce the 925 clearest AFM images. The hydrophobic cleavage plane of graphite orients the adsorbed 926 surfactant structures more strongly than any other substrate, and hemi-cylindrical structures 927 are formed for surfactant chains with more than 10 carbon atoms, smaller chains result in a 928 laterally featureless adsorbed layer. The hydrophilic, crystalline mica substrate also orients 
929 the adsorbed surfactant structures, but not as strongly as graphite. The alkyl trimethyl-

930 ammonium surfactants form cylindrical admicelles, which were stable at relatively high

931 ionic strength, but less stable at low ionic strength. The morphology of adsorbed gemini

932 surfactants on mica depend strongly on the geometry of the surfactant monomer. On silica

933 surfactants are less strongly oriented, due to a lower surface charge and the amorphous state

934 of the substrate. The adsorbed configuration of C16TAB was concentration dependent, with

935 short rods at $0.9 * \mathrm{CMC}$ and worms at $10 * \mathrm{CMC}$. Similar structures have been observed for

936 C14TAB and C12TAB on silica. For C16TAC, only spheroidal structures were present. In

937 all cases there was little or no long range ordering of the adsorbed aggregates. By

938 sequentially increasing the substrate hydrophobicity the structure of adsorbed nonionic

939 surfactants can be changed in a controlled manner from diffuse adsorbed micelles on the

940 least hydrophobic interface, to densely packed micelles, to a bilayer, to a monolayer on the

941 most hydrophobic interface. The information gained by AFM, fluorescence quenching and

942 neutron reflectivity is discussed and with this knowledge, kinetic data obtained from

943 ellipsometry and optical reflectometry, are analyzed. In the summary the likely mechanisms

944 of adsorption along the four-regions isotherm are proposed.

945 Zhmud and Tiberg [130] discuss applications of modern techniques (tensiometry, 946 ellipsometry, photon correlation spectroscopy, and neutron reflectivity) with emphasis on

947 the physical principles and theoretical aspects related to the adsorption and desorption

948 kinetics, interfacial structure development, wetting enhancement, and interfacial dynamics.

949 The review includes mathematical derivations that demonstrate how raw data can be 950 transformed into sought layer characteristics. After the discussion of the techniques the 951 intermolecular interactions in aggregated surfactant complexes are discussed and 952 experimental studies on the dynamics and structure of adsorbed surfactant layers are briefly 953 reviewed. The discussion of the isotherm for ionic surfactants is somewhat confusing 954 because two surface association concentrations are considered, the hemi-micelle 
955 concentration and the critical surface association concentration. Although different names

956 have been used for the concentration at which aggregation starts, there is for a given system only one concentration where the four-region isotherm steeply increases. In the last part of 958 the review wetting enhancement by surfactants and the effect of surfactants on the damping 959 of capillary waves are discussed.

A topical review on in-situ surface-enhanced infrared and non-linear vibrational sum-frequency spectroscopy in relation to a better understanding of adsorption at mineral-water interfaces is presented by Schrödle and Richmond [131]. Particular consideration is given to organic adsorbates including surfactants. The authors conclude that 964 non-linear optical methods greatly extend the well-established linear IR techniques and 965 provide many opportunities to advance our understanding of the structure and dynamics of 966 mineral-water interfaces. This will be true, but, as the material is quite complex, help of an expert will be required for the data analysis.

Many of the experimental results discussed in the above reviews are obtained on graphite, 969 mica, quartz and silica and these surfaces are different from the metal oxide surfaces. 970 Reviews that put more emphasis on metal oxide surfaces are those of Paria and Khilar [132] 971 and Zhang and Somasundaran [133]. Paria and Khilar discuss the four-regions isotherms of 972 ionic surfactants on oppositely charged metal oxides, and pay special attention to region IV, 973 that occurs for surfactant concentrations > CMC, where some anomalies have been 974 observed. Influences of molecular structure, temperature, salt concentration that are very 975 important in surfactant adsorption are reviewed for both nonionic and ionic surfactants and 976 the state of aggregation of the adsorbed surfactants is discussed on the basis of AFM and 977 fluorescence measurements. Also the adsorption behavior and mechanism of different 978 mixed surfactant systems (anionic-cationic, anionic-nonionic and cationic-nonionic) are 979 reviewed. Mixtures of surface-active materials can show synergistic interactions, which can 980 be manifested as enhanced surface activity, spreading, foaming and detergency. 
The review of Zhang and Somasundaran [133] is concerned with adsorption of single

982 surfactants as well as mixtures of various types and provides lists of systems (surfactant / surface / method) that have been studied for both ionic and nonionic surfactants. Also zwitterionic surfactants are briefly discussed. The information derived from the isotherms, combined with fluorescence spectroscopies, electron spin resonance (ESR), Raman spectroscopies, small angle neutron scattering, neutron reflectivity and AFM, has been used to discuss the structural properties of the surface aggregates along the isotherms. Compared to the adsorption of single surfactants, adsorption of mixtures of anionic- cationic, anionicnonionic, cationic- nonionic, cationic- zwitterionic and nonionic- nonionic types generally exhibit synergy at interfaces. To predict the shape of mixed aggregates of nonionic surfactant mixtures a new model of the critical packing parameter is derived that takes into account the mole fractions of adsorbed surfactants.

\subsection{Surfactant adsorption and charge regulation}

In the next sections the adsorption isotherms of ionic surfactants to rutile $\left(\mathrm{TiO}_{2}\right)$ and silica measured at constant $\mathrm{pH}$ and different background 1-1 electrolyte concentrations will be critically examined, together with the adaptation of the surface charge as a result of the surfactant adsorption. Results for rutile are taken from [118, 134-136], those for silica from $[137,138]$. It will be shown that these surfaces behave differently with respect to surfactant adsorption. A strictly constant $\mathrm{pH}$ allows the calculation of the surface charge regulation (adaptation) and measurements at different ionic strength at constant $\mathrm{pH}$ can be of help with unraveling the orientation of the adsorbed surfactant. In order to keep the $\mathrm{pH}$ constant, the $\mathrm{pH}$ is regularly checked during the course of adsorption and restored to its initial value. The amounts of alkali or acid to restore the $\mathrm{pH}$ are used to calculate the proton charge adaptation of the surface. The surface charge adjustment is important in itself (in many studies the effect of a change in surface charge is simply ignored), but it can also help to create a 
1007 picture of the adsorption behavior. It is also required for full understanding of specific

1008 co-ion and counterion effects on surfactant adsorption. A combination of surfactant

1009 adsorption and surface charge (adjustment) results makes it possible to differentiate between

1010 (i) adsorbed surfactant molecules with the head groups adjacent to the surface ('head-on'

1011 adsorption) which may occur with a counterbalancing (de)protonation of the surface, and

1012 (ii) molecules with an oppositely orientation, i.e. with their head group at the solution side

1013 ('head-out' adsorption) which will not affect the surface charge. To discriminate between

1014 different aggregate structures monolayer type aggregates of head-on adsorbed molecules

1015 will be called 'teepee'-micelles [128] and local bilayer type structures (head-on + head-out

1016 adsorbed molecules) ad-micelles. Knowledge about the prevailing molecular orientation and

1017 or aggregate structure is of direct importance for wetting and stability behavior of the

1018 particles [25, 127].

1019

1020

5.3. Ionic surfactant adsorption on rutile.

1021 Rutile is chosen because it allows studying anionic surfactant adsorption on positive

1022 rutile at the acidic side of the $\mathrm{pH}$ of zero net proton charge $\left(\mathrm{pH}<\mathrm{pH}_{\mathrm{pznpc}}\right)$ and cationic

1023 surfactant adsorption on negative rutile at the alkaline side $\left(\mathrm{pH}>\mathrm{pH}_{\mathrm{pznpc}}\right)$. Rutile is not of

1024 direct importance for soil systems but at the same ionic strength the adsorption behavior of

1025 surfactants on rutile is very similar to that on aluminum oxides and hematite when $\Delta p H=$

$1026 \mathrm{pH}-\mathrm{pH}_{\mathrm{pznpc}}$ is considered as parameter that characterizes the charge density [134, 139-141].

1027 At similar $\mathrm{pH}$ values the adsorption isotherms and admicelle structures of a given surfactant

1028 on different metal oxides surfaces will strongly depend on $\mathrm{pH}-\mathrm{pH}_{\mathrm{pznpc}}$, i.e., on the type of

1029 metal oxide surface [142]. Nonionic adsorption on rutile will not be discussed.

1030 In Fig. 15 adsorption isotherms of sodium nonyl-benzene-sulfonate (SNBS) on rutile at

1031 pH 4.1 and three salt concentrations are depicted as log-log (a) and lin-log (b) plots [134].

1032 In region I the coverage is too low for the adsorbed molecules to interact with each other, 
1033 the slope is about unity. Mutual attraction starts in region II, where the coverage is still very

1034 low: roughly between 0.03 and $3 \%$ of the maximum coverage. The concentration at the 1035 transition point between region I and II is the critical surface association concentration or 1036 CSAC. A straight line with a slope of about four fits the data points in region II, indicating a 1037 strongly positive cooperativity of the adsorption and proof for surfactant self-assembly. The 1038 slope in region III is considerably lower than that of region II and it depends notably on the 1039 salt concentration. It is less than unity for low salt concentrations and somewhat higher than 1040 unity for $0.1 \mathrm{M}$ salt. Salt addition promotes the adsorption in region III by screening the 1041 head group repulsion. The transition between regions II and III is more pronounced for low 1042 salt concentrations, which also indicates that at low salt concentrations the repulsion 1043 between head groups becomes important in region III. The CMC marks the transition to 1044 region IV, where the plateau is reached.

1045 Due to the fact that isotherms at three salt concentrations are plotted another important 1046 phenomenon occurs: the three isotherms at different salt concentration intersect in a 1047 common intersection point or CIP. The presence of such a CIP was first noted by De Keizer 1048 et al. [143] who also showed that in the absence of specific adsorption of other ions and/or 1049 activity effects the CIP should correspond to the IEP of the particles covered with surfactant. 1050 In Fig. 15(b), the lin-log plot, the strong cooperativity present at low coverage is evident at 1051 low salt concentrations as a weak shoulder in the isotherms. The lin-log isotherms show 1052 much better than the log-log isotherms that the 'bulk' of the adsorption occurs in region III. 1053 Also the position of the (near) CIP is clearly shown. The plateau values reached in region IV 1054 are about the same for the different salt concentrations.

1055 The adsorption of SNBS and surface charge of rutile are shown in Fig. 16 as function of 1056 the surfactant concentration at three $\mathrm{pH}$ values and two salt concentrations [134]. To 1057 facilitate the comparison the surface charge is divided by F, the Faraday, so that proton 1058 excess, $\Gamma(0)$, and adsorbed amount of surfactant, $\Gamma$ s (SNBS), are both expressed in $\mu \mathrm{mol} / \mathrm{m}^{2}$. 
1059 Information about the structure of the adsorbed layer can be obtained by comparing $\Gamma(0)$

1060 and $\Gamma$ s. For a given pH, see e.g. pH 4.1 (Fig. 16, panel a and b), head-on adsorbed surfactant

1061 ions may either have displaced adsorbed $\mathrm{Cl}^{-}$ions or caused the formation of new proton

1062 charges at the surface to counter balance the surfactant charge and the latter increases $\Gamma(0)$.

1063 Head-out adsorption will not affect the surface charge. Therefore, as long as the surface

1064 charge adjusts to the surfactant adsorption head-on adsorption occurs. Initially $\Gamma(0)>$

$1065 \Gamma$ (SNBS) because the surface charge is already present (best seen at $0.1 \mathrm{M} \mathrm{NaCl}$ ) but at

1066 somewhat larger surfactant concentrations $\Gamma(0)<\Gamma$ (SNBS). Beyond the intersection point

1067 the difference between $\Gamma \mathrm{s}$ and $\Gamma(0)$ is due to head-out adsorption plus some head-on

1068 adsorption of molecules that are accompanied by a counter ion; therefore, head-out

1069 adsorption is about proportional to $\Gamma \mathrm{s}-\Gamma(0)$ but not necessarily equal to this difference. The

1070 proton uptake due to surfactant adsorption is the strongest at low surfactant adsorption and

1071 low salt concentrations: with almost every adsorbing surfactant molecule a proton is

1072 adsorbed, indicating that all surfactant ions will be adsorbed head-on. At higher salt

1073 concentrations the initial surface charge is higher, therefore the initial slope of the $\Gamma(0)$

1074 curve is less, surfactant ions cause displacement of $\mathrm{Cl}^{-}$ions at the surface by $\mathrm{NBS}^{-}$ions and

1075 proton adsorption. At high amounts adsorbed the surface charge becomes almost

1076 independent of the salt concentration: the surfactant is responsible for the screening of the

1077 surface charge. Very similar results are obtained at $\mathrm{pH} 5.1$ and 6.0. At pH 6 the pure rutile

1078 surface is slightly negative $\left(\mathrm{pH}_{\mathrm{pznpc}}\right.$ rutile 5.85); however, the specific affinity of the head

1079 group for the surface is sufficient to overcome the coulomb repulsion and the surfactant

1080 adsorption induces a positive charge on the surface. Therefore, only positive surface charges

1081 are observed once surfactant is present, and the two curves coincide at low surfactant

1082 concentrations.

1083 In general, the proton adsorption tends to level off after the CIP in the isotherms. The

1084 CIP corresponds for SNBS with the IEP and with the point where the surface charge and the 
1085 surfactant charge balance, the equivalence point. The strong surface charge adjustment

1086 before the CIP in combination with the observed steep slope of region II of the isotherms

1087 indicates that teepee-micelles are formed at the surface. Teepee-micelle formation is

1088 promoted by the 'flexibility' of the surface charge. Changes in surface charge due to SNBS

1089 adsorption still occur beyond the CIP. This demonstrates that the head-on adsorption is not

1090 completed at the CIP. The substantial difference between $\Gamma$ s and $\Gamma(0)$ beyond the CIP

1091 indicates that a large part of the additionally adsorbing molecules is oriented head-out: after

1092 the CIP the formation of admicelles is dominant. The rate of transition from teepee- to

1093 ad-micelles depends strongly on the salt concentration; the higher the salt concentration the

1094 sharper becomes the transition. The SNBS adsorption around the CMC depends only

1095 weakly on the salt concentration, increasing the salt concentration increases the adsorption

1096 somewhat due to the better screening of the head group charges.

1097 Comparing the behavior at the three $\mathrm{pH}$ values (Fig. 16) it can be observed that the $\mathrm{pH}$

1098 effect on the SNBS adsorption is most strongly at the initial part of the isotherm (region I

1099 and II): the smaller $\Delta \mathrm{pH}=\mathrm{pH}^{\mathrm{pH}} \mathrm{p}_{\mathrm{pznpc}}$ is, the lower is the affinity of the surfactant for the

1100 surface. However, the pH may also affect the admicelle structure because an increase in

1101 surface charge density can lead to a reduction in the effective headgroup area of the

1102 adsorbed surfactant and this may decrease the critical packing parameter [142]. Further

1103 adsorption studies on positively charged rutile with four sodium p-dodecylbenzene

1104 sulfonate isomers (3SDBS, ISDBS, 5SDBS, 6SDBS) [135] to study the effects of aliphatic

1105 chain length and architecture, revealed that the adsorption increases with chain length and

1106 decreases if the point of attachment of the aliphatic chain to the hydrophilic part of the

1107 molecule is shifted from a terminal to a medial position.

1108 The adsorption on negative rutile was studied by using two pyridinium surfactants (C12,

1109 C14) and the results were compared with those of SNBS (see Fig. 14) [118, 136]. The

1110 four-regions isotherm and the CIP are found also for the anionic surfactant adsorption on 
1111 positive rutile, but for the weakly adsorbing $\mathrm{CnP}^{+}$ions the situation is considerably more

1112 complicated than for the strongly adsorbing $\mathrm{NBS}^{-}$ion. Due to the relatively weak $\mathrm{CnP}^{+}$

1113 adsorption, the competition with the salt cations $\left(\mathrm{K}^{+}\right)$becomes noticeable and the 'CIP' is

1114 no longer 'sharp', the IEP shifts with the salt concentration and differs from the 'CIP'.

1115 However, the 'CIP' remains the transition point for tepee- to ad-micelle formation. For more

1116 details and a further discussion of surfactant adsorption on rutile or the metal hydroxides in

1117 general the quoted references should be consulted.

1118

\subsection{Ionic surfactant adsorption on silica}

1120 The adsorption of cationic surfactants on silica (negative) is far greater than the adsorption

1121 of anionic surfactants. Nevskaia et al. [144] have shown that small amounts of anionic

1122 surfactants $\left(\mathrm{C}_{9} \mathrm{H}_{19}\right.$-phenol- $\left(\mathrm{OCH}_{2}-\mathrm{CH}_{2}\right)_{n}-\mathrm{SO}^{-} \mathrm{Na}^{+}$, with $\mathrm{n}=4$ and 10) could be adsorbed on

1123 quartz but the relevance is very limited. Therefore, no further attention will be paid to anionic

1124 adsorption to silica.

1125 The adsorption isotherms for C12PC on Aerosil, measured at $\mathrm{pH} 9$ and two salt 1126 concentrations are shown in Fig. 17(a) as log-log plot and in Fig. 17(b) as lin-log plot [137].

1127 The isotherms at low and high salt concentrations have different shapes. At low salt

1128 concentration the adsorption shows a 'hesitation' in the both the lin-log and log-log plot. This

1129 is usually observed for adsorption of cationic surfactants on silica in the absence of salt or at

1130 low salt concentrations [145-148]. At both low and high salt concentrations four regions may

1131 be distinguished in the log-log plots and for low salt concentration the different regions can

1132 also be observed in the lin-log plot. The slope in region I of the C12PC isotherms is equal to

1133 unity within experimental error. In this region specific and coloumbic attraction between

1134 surface and surfactant ions take place, but the adsorbed molecules don't interact with each

1135 other. In region II, the slope of isotherms, measured at low salt concentration, is less than

1136 unity. This demonstrates that surfactant self-assembly still does not occur! Self-assembly is 
1137 counteracted by mutual head group repulsion and by the partial hydrophobicity of the

1138 pyrogenic silica surface that interacts with the surfactant tails by hydrophobic attraction. This

1139 behavior deviates strongly from that observed for adsorption of C12PC on rutile [118, 136]

1140 and in general from surfactant adsorption on the crystalline metal oxides. On silica surfactant

1141 molecules adsorb, at low salt concentration, without appreciable lateral interaction up to the

1142 end of region II. In the same concentration range the surface charge adapts strongly to the

1143 surfactant adsorption. Therefore, the mutual repulsion between the headgroups will be modest

1144 and most likely the tail-surface interaction is the main reason for the absence of self-assembly.

1145 Evidence for tail-surface interaction was obtained from both comparison of the effect of

1146 C16TAB and tetramethyl-ammonium bromide on the charging behavior of Aerosil [138] and

1147 theoretical calculations using the SCFA model [137]. The surfactant concentration has to

1148 increase to about $0.1 * \mathrm{CMC}$ before region III begins and the aggregation starts. Due to lateral

1149 hydrophobic attraction the slope of the isotherm in region III is steeper than that in II and the

1150 CSAC is located at the transition of region II to region III.

1151 Increasing the salt concentration increases the slope of region II due to the higher initial

1152 surface charge and a better screening of the lateral head group repulsion. At $0.1 \mathrm{M}$ salt the

1153 slope of region II is larger than that at region I; therefore, the CSAC now occurs at the

1154 transition from region I to region II. A relatively high salt concentration leads to a relatively

1155 high surface charge due to the presence of many counterions close to the surface, and this

1156 reduces the surface hydrophobicity. Consequently, not only the mutual head group repulsion

1157 is diminished, but also the tail-surface attraction, therefore surfactant self-assembly occurs at

1158 a much earlier stage than at low salt concentration. The slope in region III depends only

1159 weakly on the salt concentration and is now smaller than in region II. The transition to region

1160 IV is observed at the CMC. An increase in the salt concentration leads to a rise of the plateau

1161 value and small decrease of concentration (CMC), where plateau is reached: diminishing the

1162 lateral repulsion allows a closer packing of the molecules and/or a higher aggregate density. 
1163

1164

1165

The isotherms show a CIP in region II, the concentration at which the intersection occurs, corresponds well with the point where the surfactant charge and the surface charge balance, the equivalence point. The fact that CIP and equivalence point coincide is direct evidence that the binding of $\mathrm{C}_{12} \mathrm{P}^{+}$to Aerosil is much stronger than that of $\mathrm{K}^{+}$. The much stronger binding of C12PC to silica than to rutile $[118,136]$ is also likely due to the tail-surface affinity in the case of silica. The reasons for the CIP are the same as those mentioned for SNBS adsorption on rutile. The difference with the SNBS-rutile case is that the CIP is located in region II instead of in region III of the isotherm.

The effect of C12PC adsorption on the surface charge of Aerosil at pH 9 is shown in Fig. 18 for two salt concentrations $(0.001 \mathrm{M}$ and $0.1 \mathrm{M} \mathrm{KCl})[138,149]$. To allow easy comparison of the adsorbed amount of surfactant, $\Gamma$ s, and the surface charge the latter is divided by $\mathrm{F}$, the Faraday, an indicated as, $\Gamma(0)$; they are both expressed in $\mu \mathrm{mol} / \mathrm{m}^{2}$. The maximum change in surface charge occurs at low surfactant and low salt concentrations, where the adsorption and surface charge isotherms almost coincide. It means, that at low salt concentrations with almost every adsorbing surfactant ion a proton is desorbed and practically all adsorbed surfactant ions will adsorb head-on. After compensation of the surface charge by surfactant (the equivalence point) the surface charge continues to increase weakly whereas the surfactant adsorption increases substantially. This indicates that locally adsorption of surfactant in head-out position starts, i.e. head group directed to the solution. The weak increase of $\Gamma(0)$ shows that some of the additionally adsorbing molecules are still head-on adsorbed. On top of this some other surfactant ions may adsorb head-on if they are accompanied with a counterion. In general, one may conclude that small admicelles are formed around the initially head-on adsorbed (isolated) molecules. This representation of the surface aggregate corresponds with that suggested by Zhu et al. [150, 151].

At high salt concentration the changes in surface charge due to the surfactant adsorption are much less, evidently $\mathrm{C} 12 \mathrm{P}^{+} / \mathrm{K}^{+}$exchange is important. The high salt concentration 
1189 screens the surface charge fairly well and it is difficult for the surfactant to start its

1190 adsorption. Once the adsorption starts it increases strongly over a rather small concentration

1191 range and a sharp intersection point with the $\Gamma(0)$ isotherm is found. This equivalence point

1192 corresponds with the CIP of the C12PC isotherms. Before the equivalence point C12P ${ }^{+}$

1193 adsorption occurs by exchange of $\mathrm{K}^{+}$, beyond the equivalence point the adsorbing surfactant

1194 ions bring their 'own' counter ions to compensate their charge. These adsorption

1195 mechanisms have been indicated before by Bijsterbosch [148]. The super-equivalent

1196 adsorption indicates admicelle (head-on + head-out adsorption) formation. The effect of a

1197 high salt concentration is twofold: (1) before the CIP teepee-micelles are formed (steep

1198 slope of region II of the log-log isotherm) and (2) the transition from head-on adsorbed

1199 molecules to admicelles becomes very sharp. In summary, below the CIP head-on

1200 adsorption occurs: at low salt concentration as isolated molecules, at high salt concentration

1201 as teepee-micelles, above the CIP head-out adsorption and ad-micelle formation occurs at

1202 all salt concentrations. Increasing the salt concentration increases the packing density of the

1203 adsorbed surfactants, but the values of the $\Gamma(0)$ at the CMC hardly depend on the salt

1204 concentration. This indicates that the admicelles become more asymmetric and that around

1205 the CMC the screening of the surface charge is almost entirely done by surfactant.

1206 Goloub et al. [137] also studied the adsorption behavior of C16PC and the effect of the pH

1207 on both C12PC and C16PC; in general the behavior for C16PC is very similar but the

1208 isotherms are shifted to lower concentrations, which makes it difficult to study the lower

1209 regions of the four-regions isotherms. Lowering the $\mathrm{pH}$ to 7 or 5 also makes the study of the

1210 isotherm more difficult because less adsorption occurs when the negative surface charge is

1211 relatively low. For a further discussion the original papers should be consulted.

1212 The experimental results for both rutile and silica have also been compared with

1213 calculations using the Self-Consistent-Field Adsorption (SCFA) model [25, 134, 135, 137,

1214 152, 153] (see also section 7) both SCFA theory and experiments show that the course of the 
1215 isotherms is dependent on the salt concentration and the charge characteristics of the surface.

1216 Also the SCFA model isotherms obtained at different salt concentrations show a CIP, and also

1217 here the CIP marks the point where the coulombic interaction vanishes and the surfactant

1218 orientation of the newly adsorbing molecules changes form mainly head-on to predominantly

1219 head-out. Although the SCFA theory is not perfect, it definitely can help to understand

1220 surfactant adsorption and to design experiments to gain further insight in the adsorption

1221 behavior.

1222 The surfactant orientations in the adsorbed layer as deducted from the present analysis 1223 relate very well to experimentally observed maximums in hydrophobicity [154-157] and 1224 flotation recovery [158, 159] of particles as a function of surfactant concentration: at low 1225 adsorbed amounts the particles are hydrophobized, whereas at high adsorbed amounts the 1226 presence of head groups at the solution side make the particles more hydrophilic again [1].

1227 The results also indicate that around the CIP the colloidal stability will be at its minimum, not 1228 only because the particles plus adsorbed layer are uncharged, but also because the particles 1229 are hydrophobized [127].

1230

\subsection{Nonionic surfactant adsorption on silica}

1232 The adsorption of nonionic surfactants of the oligo(oxyethylene) n-alkyl ether 1233 [Cn(EO)m] or oligo(oxyethylene) n-alkylphenyl ether [CnФ(EO)m] type at the solid/aqueous 1234 interface has been the subject of much experimental and theoretical research, see the review 1235 of Tiberg et al. [129] and the cited references. The vast majority of adsorption studies of 1236 nonionic surfactants have been performed on silica. A classical set of adsorption isotherms of 1237 nonionic surfactants with different polar head group is shown in Fig. 19 [160]. From Fig. 19 it 1238 follows that the affinity increases with EO chain length, but that the adsorption level around 1239 the CMC strongly decreases.

1240 The main experimental evidence shows that the adsorbed nonionic surfactants aggregate 
1241 strongly on hydrophilic surfaces such as silica. Oxyethylene (EO) groups bind initially to the

1242 silanol groups by hydrogen bonding and this initiates strong adsorption at the CSAC through

1243 self-assembly. Contrary to ionic surfactants, nonionics do not readily make hydrophilic

1244 particles very hydrophobic because the head-on adsorption (monolayer-type) is relatively low.

1245 With limited EO chain length pronounced adsorption on silica starts at the CSAC, because the

1246 affinity of the EO segments to the surface is relatively weak. In the case of surfactants having

1247 short EO chains relative to the length of the aliphatic chain part, bilayers are formed on the

1248 surface and when the EO chain is long the adsorbed layer consists of small admicelle type

1249 aggregates.

1250 Denoyel and Rouquerol [161] have shown that different silicas (Spherosil, Aerosil and 1251 quartz) show quite different adsorption levels $\left(\mu \mathrm{mol} / \mathrm{m}^{2}\right)$, but that the adsorption mechanism

1252 is the same on the silicas despite the great differences in adsorption. The initial anchoring of

1253 molecules is exothermal on the three samples (but with a different magnitude of the

1254 corresponding enthalpy) and the subsequent surfactant aggregation is endothermal and tends

1255 for all three surfaces towards a value equal to the micellization enthalpy. This two-step

1256 mechanism involves weak hydrophobization of the surface at low surfactant concentration

1257 followed by hydrophilzation at concentrations still below the CMC. Such a transition has

1258 been confirmed for the quartz-nonionic surfactant system by contact angle measurements

1259 [162].

1260 For a good understanding of the behavior accurate experiments come in the first place,

1261 but modeling experiments can help to explain the behavior and reveal trends that govern the

1262 adsorption. SCFA model calculations [152] have been used to analyze the results of Levitz

1263 et al. [160, 163, 164] on the adsorption of a series of alkyl-phenol polyoxyethylene glycols

1264 on silica. This work was followed by a detailed experimental study of the adsorption of

1265 C12(EO)6, C12(EO)25 and the oligomer (EO)122 on different silicas [153]. To be able to

1266 apply the various techniques, different silica surfaces had to be used. To some extent this 
1267 was a disadvantage, but by comparing the results, trends emerged, and these could be

1268 studied with the $1 \mathrm{D}$ and 2D versions of the SCFA theory and in this way a better insight was 1269 gained. Recently the SCFA theory has been applied to investigate the experimentally 1270 observed $\mathrm{pH}$ and salt concentration dependence of $\mathrm{Cn}(\mathrm{EO}) \mathrm{m}$ adsorption to silica [165]. The 1271 parameter choices and some aspects of the model were refined as compared to the older

1272 SCFA modeling; the calculations contributed to detailed insight in the mechanisms of the $1273 \mathrm{pH}$ and salt effects. At high ionic strength, the solvent quality for the surfactant head groups

1274 is affected, which changes both bulk solution and adsorption behavior of the surfactant. 1275 Nonionic surfactants adsorb above the CSAC, which is a function of surfactant and surface 1276 properties. Therefore, the CSAC varies with both the ionic strength and the $\mathrm{pH}$. The model 1277 predicts that with increasing ionic strength, the CSAC will first slightly increase but then 1278 drop substantially. The charge on the surface is $\mathrm{pH}$ dependent, and as the head groups bind 1279 through H-bonding to the silanol groups, the CSAC increases with increasing $\mathrm{pH}$. The 1280 predictions follow experimental findings, which shows that the molecularly realistic SCFA 1281 model can reveal a rich interfacial behavior.

\section{Surfactant adsorption to clays}

\subsection{General aspects and outline}

An analysis of surfactant adsorption on clays is faced with several difficulties: (1) clay surfaces have basal plates and edges that make the surfaces patchwise heterogeneous, (2) for swelling clays surfactants may also intercalate between the layers and (3) the anionic surfactants tend to precipitate in the presence of multivalent cations. The patchwise nature of the clay surface is a fact that has to be considered in all cases of surfactant adsorption to clays. For expanding clays the adsorption is far more complicated than for non-expanding clays. For instance, the adsorption of anionic (SDS), nonionic (Triton X100) and cationic 
1293 (C18TAB) surfactants did not modify the X-ray diffraction diagrams of kaolinite and

1294 sepiolite (non-expanding clay types), but the interlayer space of montmorillonite (expanding

1295 clay type) increased through nonionic and cationic surfactant adsorption [166]. When the

1296 interlayer spacing expands upon surfactant adsorption the adsorption capacity becomes a

1297 function of the amount adsorbed and this leads to complex shaped adsorption isotherms that

1298 do not follow classical behavior. A further complication with anionic surfactant adsorption

1299 is that cations bound to the clay surface may exchange with the counterions of the anionic

1300 surfactant and this can lead to precipitation of the anionic surfactant, especially when the

1301 clay charge is (partially) compensated by multivalent cations [167]. Furthermore, at

1302 relatively low $\mathrm{pH}$ leaching of $\mathrm{Al}^{3+}$ ions from the clay may occur and can be promoted by the

1303 presence of anionic surfactants through $\mathrm{Al}^{3+}$ complexation with the anionic surfactant

1304 followed by surfactant precipitation [168-170]. Any precipitation of the surfactant obscures

1305 the pure adsorption results.

1306 The most studied non-expanding clay with respect to surfactant adsorption is kaolinite

1307 and cat-, an- and nonionic surfactant adsorption to kaolinite will be discussed in the next

1308 sections, with emphasis to cationic surfactants. Subsequently, surfactant adsorption to

1309 montmorillonite will be discussed at surfactant concentrations up to the CMC. The situation

1310 of surfactant binding to expanding clays to produce organo-clays for various specific

1311 applications is a field in itself. For reviews of this field we refer to [171-174] and to some

1312 original papers [175-178].

1313

$1314 \quad 6.2$ Cationic Surfactant adsorption on kaolinite

1315 De Keizer et al. [143, 179, 180] have made a very thorough study of the adsorption of 1316 cationic surfactant adsorption (C16TAB and C12PC) on kaolinite (Sigma Chemicals) by 1317 investigating the adsorption behavior at different $\mathrm{pH}$ values and salt concentrations as a 1318 function of temperature. Adsorption isotherms (lin-log) of C16TAB and C12PC at different 
1319 electrolyte concentrations show a well-defined CIP where the adsorption is independent of

1320 the electrolyte concentration [143, 179], see Fig. 20. The CIP coincided with the IEP of the

1321 particles with adsorbed surfactant, indicating that at this point the surface charge is

1322 compensated by the surfactant charge, including any counter-charge that may be present

1323 within the slip layer. The presence of a CIP and the correspondence with the IEP seems to

1324 be a general property for adsorption of organic ions on a surface with an opposite charge.

1325 Very similar behavior occurred for the surfactant adsorption on silica and rutile (metal

1326 oxides). Also for kaolinite head-out (second layer) adsorption driven by hydrophobic

1327 attraction starts after the CIP. This view is supported by the effect of the temperature on the

1328 adsorption. Below the CIP, adsorption is independent of temperature, whereas after the CIP

1329 it has a maximum as a function of temperature. With increasing temperature the adsorption

1330 changes from endothermic to exothermic, as for micellization. The CIP occurred for both

1331 surfactants at about $32 \mu \mathrm{mol} / \mathrm{g}$, roughly $40 \%$ of the maximally attained value. In the

1332 CIP=IEP the charged surfactant groups just compensate the surface charge. Therefore, the

1333 above value may also be compared with the cation-exchange capacity (CEC). The CEC

1334 according to the silver-thiourea method was $57 \mu \mathrm{mol} / \mathrm{g}$ (exchange on plates plus edges),

1335 while the CEC with the ammonium acetate method at $\mathrm{pH} 7$ was $30 \mu \mathrm{mol} / \mathrm{g}$ (cation exchange

1336 on the plates). This provided evidence that C16TAB and C12PC adsorption took place

1337 essentially on the plate surfaces.

1338 In part 3 of the study [180] the investigations were complemented with 1339 micro-calorimetric measurements of the adsorption enthalpies of C12PC on Na-kaolinite at 1340 two electrolyte concentrations (5 and $100 \mathrm{mM} \mathrm{NaCl}$ ) and three temperatures (6, 20 and $134160^{\circ} \mathrm{C}$ ). Curves of cumulative adsorption enthalpies against amount adsorbed show a break 1342 around the CIP adsorption. The curves are about linear both before and after the break, and 1343 result in partial molar adsorption enthalpy values that are independent of surface coverage, 1344 confirming that the surface is homogeneous with respect to the C12PC adsorption. This is 
1345 additional evidence that the surfactant is hardly adsorbed on the edges. The isotherms are

1346 independent of temperature up to CIP coverage, implying a zero isosteric adsorption

1347 enthalpy, but upon formation of the second layer (head-out mode) the adsorption has a

1348 maximum at about $23{ }^{\circ} \mathrm{C}$, implying a transition from an endothermic to an exothermic

1349 process. The calorimetric adsorption enthalpies change sign at $\mathrm{T} \approx 24-30{ }^{\circ} \mathrm{C}$. In this respect,

1350 the adsorption process is very similar to micellization [181]. The electrolyte concentration

1351 has a minor effect on the adsorption enthalpies of the first (head-on adsorption) and second

1352 (head-out adsorption) layers. The directly measured heats agree qualitatively with the

1353 isosteric heats.

1354 In the last paper [180] the isotherms were also modeled with a simple two-layer model

1355 based on the Frumkin-Fowler-Guggenheim model that takes into account both the

1356 interaction with the surface and the lateral interaction, therefore the standard Gibbs energies

1357 have two contributions: $\Delta \mathrm{G}_{\text {surface }}^{0}$ and $\Delta \mathrm{G}^{0}$ lat. For the first layer it is assumed that the

1358 molecules adsorb head-on to the mineral surface with their tails at the solution side, for

1359 adsorption in the second layer the 'surface' is made-up of the tails of the first layer and the

1360 molecules adsorb head-out with their tails contacting the new 'surface'. Since the

1361 adsorption on kaolinite particles involves chemical and electrical contributions, the changes

1362 in the standard Gibbs energies can be written as $\Delta G^{0}=\Delta G_{\text {chem }}^{0}+\Delta G_{\text {el }}^{0} \Delta G_{\text {el }}^{0}$ accounts for

1363 the generic interactions due to the 'smeared-out' electrostatic potentials, $\Delta \mathrm{G}_{\text {chem }}^{0}$ contains

1364 chemical (specific), hydrophobic and 'local' electrostatic interactions (local self-atmosphere

1365 potentials). Because of their special nature and important role the hydrophobic attractions

1366 are separately indicated. The standard Gibbs energy of adsorption in contact with the

1367 surface (layer: 1 , surface: s), $\Delta \mathrm{G}_{1, \mathrm{~s}}^{0}$ can then be written as $\Delta \mathrm{G}_{1, \mathrm{~s}}^{0}=\Delta \mathrm{G}_{1, \mathrm{~s} \text {-spec }}^{0}+$

$\left.1368 \Delta \mathrm{G}_{1, \mathrm{~s} \text {-hydrophobic }}^{0}+\Delta \mathrm{G}_{1, \mathrm{~s}-\mathrm{el}}^{0}\right) ; \Delta \mathrm{G}_{1, \mathrm{~s}}^{0}$ becomes less negative with increasing electrolyte

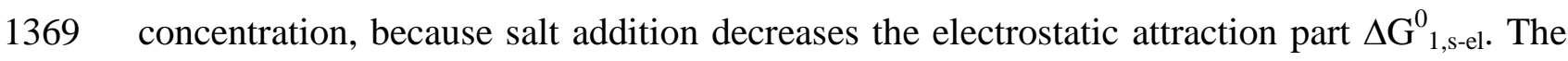

1370 effect of salt addition on $\Delta G_{1, \mathrm{~s}}^{0}$ is opposite to that on $\Delta \mathrm{G}^{0}{ }_{1 \text {,lat }}$, because for $\Delta \mathrm{G}^{0}{ }_{1 \text {,lat }}$ 
$1371 \quad\left(=\Delta G^{0}{ }_{1 \text {,lat-hydrophobic }}+\Delta G^{0}{ }_{1 \text {,lat-el }}\right)$ the salt effect is favorable (repulsion between surfactant head

1372 groups decreases). The two salt effects compensate each other at the CIP. The $\Delta \mathrm{G}_{2, \mathrm{~s}}^{0}$

1373 (second layer) values are hardly dependent on the salt level, because the second layer starts

1374 when the particle potential is close to zero (CIP $\approx$ IEP); therefore, to a good approximation,

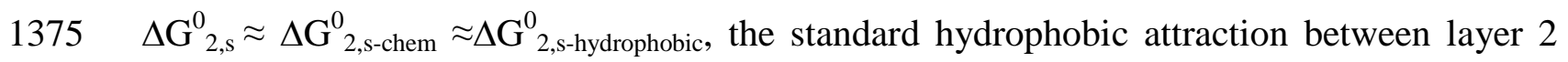

1376 and layer 1 (which is the new s). However, the lateral attraction in layer $2, \Delta \mathrm{G}_{2 \text {,lat, }}^{0}$

$1377\left(=\Delta G_{2, \text { lat-hydrophobic }}^{0}+\Delta G_{2 \text {,lat-el })}^{0}\right.$ also contributes and increasing the salt concentration from

137820 to $100 \mathrm{mM}$ causes a decrease of $\Delta \mathrm{G}^{0}$,lat-el as a result of a decrease in the electrostatic

1379 repulsion between the head groups and, in turn, this increases net attraction and hence the

1380 adsorption.

1381 The sum $\left(\Delta G_{1, \mathrm{~s}}^{0}+\Delta \mathrm{G}_{1 \text {,lat }}^{0}\right)$ turns out to be hardly temperature dependent. This shows that

1382 the independence of the temperature of the adsorption in the first layer is caused by internal

1383 compensation. As a result, according to the Gibbs-Helmholtz equation, $\Delta \mathrm{H}_{1, \mathrm{~s}}^{0} \approx 0$. However,

1384 for the second layer the sum $\left(\Delta \mathrm{G}^{0}{ }_{2, \mathrm{~s}}+\Delta \mathrm{G}^{0}{ }_{2 \text {,lat }}\right)$ is smaller at $20{ }^{\circ} \mathrm{C}$ than at $60{ }^{\circ} \mathrm{C}$, in line with

1385 the exothermic nature of the process over this temperature range. At $20^{\circ} \mathrm{C}$ the adsorption

1386 enthalpies, $\Delta \mathrm{H}_{1, \mathrm{~s}}$, are much smaller than $\Delta \mathrm{G}^{0}{ }_{1, \mathrm{~s}}$ indicating that entropic contributions dominate

1387 at both electrolyte concentrations. Apparently, for both layers the enthalpies of hydrophobic

1388 bonding of the tails and those due to interactions of the head group and/or change of hydration

1389 at the surface compensate. At $60^{\circ} \mathrm{C}$ the entropy has decreased, but the (exothermic) enthalpy

1390 renders the standard Gibbs energies virtually temperature independent. This is another

1391 example of enthalpy entropy compensation which is characteristic for hydrophobic bonding

1392 and which has also been found for the solubility of alkanes in aqueous solution [182, 183].

1393 Xu and Boyd [184] have obtained very similar C16TAC isotherms (lin-log) on kaolinite

1394 (Source Clay Minerals Repository, University of Missouri, Columbia) at pH 6.5 and two

1395 salt concentrations (2 and $42 \mathrm{mM}$ ), see Fig. 21. The (C)IP of the isotherms is found at about

$139620 \mu \mathrm{mol} / \mathrm{g}$ (0.5CEC). The reported CEC value at $\mathrm{pH} 6.5$ was $40 \mu \mathrm{mol}_{\mathrm{e}} / \mathrm{g}$; this would imply 
1397 that about $50 \%$ of the CEC is due to the plate surfaces. Although the authors measured

1398 electrophoretic mobilities and dispersion stability of montmorillonite, it is a pity that they

1399 did not state such results for kaolinite, because this might have shown the correspondence

1400 of their (C)IP with the IEP or the minimum in dispersion stability.

1401 Wang et al. [185] studied the adsorption of a series of alkyl-trimethylammonium

1402 bromides $\left(\mathrm{RR}_{1} \mathrm{~N}\left(\mathrm{CH}_{3}\right) \mathrm{Br}\right)$ with different chain lengths on sodium kaolinite (Source Clay

1403 Minerals Repository, University of Missouri, Columbia) by micro-electrophoresis and

1404 adsorption isotherms. The isotherms (lin-log) were measured over a relatively large

1405 concentration range and have a similar shape than those measured by de Keizer et al. [143],

1406 Mehrian et al. [179] and Xu and Boyd [184]. At very low concentrations the adsorbed

1407 amount increased only slowly with increasing surfactant concentration; the second stage of

1408 adsorption occurred at higher surfactant concentrations below the CMC and a sharp increase

1409 of adsorption was observed. Adsorption in the second stage is accompanied by a sharp

1410 change in the $\zeta$-potential, charge reversal and second layer formation. The third region is

1411 located around the CMC with a constant adsorption above the CMC, where the monomer

1412 concentration and the adsorption remain constant. For all surfactants the amount adsorbed at

1413 the IEP is about $10-12 \mu \mathrm{mol} / \mathrm{g}$. Though kaolinite came from the same source as that of $\mathrm{Xu}$

1414 and Boyd [184], this value is about half the value reported by $\mathrm{Xu}$ and Boyd [184]. The CEC

1415 of the kaolinite is not reported in the paper so no further comparison can be made between

1416 the adsorption at the IEP and the CEC. An increase in the number of $\mathrm{CH}_{2}$ groups in the

1417 shorter alkyl chain of the double chain surfactant, or the introduction of another kind of

1418 alkylgroup (phenyl group), was found to have much less effect on the adsorption behavior

1419 than changing the length of the alkyl chain in the single-chain surfactants. This corresponds

1420 with the trend in CMCs and with the findings of Böhmer et al. [135] for surfactant

1421 adsorption on rutile. Intra-molecular association reduces the effective hydrophobicity of the

1422 shorter chains in the double-chain surfactants. 
1423 A further study on chain architecture and adsorption on kaolinite was made by Qi et al.

1424 [186] who compared the adsorption of C16TAB with a C16-2-C16 gemini surfactant of

1425 similar structure (2 $\mathrm{CH}_{2}$ segments as spacer) at $\mathrm{pH} 6$ and a ionic strength of about $0.02 \mathrm{M}$

1426 salt. Compared to C16TAB the affinity of the gemini surfactant was higher and the isotherm

1427 was steeper, but the maximum adsorptions in $\mu \mathrm{mol} / \mathrm{g}$ at the CMC were rather similar. The

1428 maximum adsorption was about 1.4CEC, so well above the charge compensation point,

1429 which provides evidence for the presence of the second layer.

1430 An alternative approach to detect the onset of the head-out adsorption and the ratio

1431 head-out / head-on adsorption (maximum adsorbed amount in the second 'layer' relative to

1432 that in the first 'layer') has been introduced by Li et al. [187-189]. The somewhat simplified

1433 notion of the adsorption behavior is that first complete cation exchange occurs due to

1434 head-on surfactant adsorption (maximum first layer surfactant adsorption $=$ CEC) and that

1435 subsequently further surfactant is adsorbed in the head-out mode by hydrophobic attraction

1436 (second layer adsorption). In the absence of added background electrolyte, surfactant bound

1437 head-on by ion exchange will loose its counterion and the cations that were adsorbed to the

1438 negative sites on basal planes will be released. For the second layer, formed by hydrophobic

1439 attraction, the mechanism is different: it leads to head-out surfactant adsorption and these

1440 surfactant ions will still require counter ions in the diffuse layer to compensate their charge

1441 and as the surfactant counterion is the only negative ion present except for some $\mathrm{OH}^{-}$, the

1442 surfactant ion adsorption will be accompanied by adsorption of its counterion. Thus, by

1443 comparing the surfactant adsorption with the counterion release from the clay (or with the

1444 CEC) and with the surfactant counterion co-adsorption a distinction can be made between

1445 first layer and second layer surfactant adsorption. This type of investigation goes back to the

1446 classical study of Bijsterbosch [148] of the counterion binding to C16TAB and C12TAB on

1447 silica. 
Li and Bowman [187] studied kaolinite (KGa-1; Source Clay Minerals Repository,

1449 University of Missouri, Columbia, MO) and a zeolite, clinoptilolite, and observed (1) that

1450 the maximum adsorption of $\mathrm{C} 16 \mathrm{TAX}\left(\mathrm{X}=\mathrm{Br}^{-}, \mathrm{Cl}^{-}\right.$or $\left.\mathrm{HSO}_{4}^{-}\right)$was in all cases larger than the

1451 CECs of the minerals, which implied that the adsorption was in excess of ion exchange

1452 and (2) that the maximum adsorption increased in the order $\mathrm{HSO}_{4}{ }^{-}<\mathrm{Cl}^{-}<\mathrm{Br}^{-}$which showed

1453 the surfactant counterion effect for the adsorption in the second layer. At the adsorption

1454 maximum the ratio surfactant counterion adsorption / surfactant adsorption ranged from

14550.33 to 0.55 in going from $\mathrm{HSO}_{4}{ }^{-}$to $\mathrm{Br}^{-}$, which is a clear indication of the formation of a

1456 second layer and that the counterion affects the amount of surfactant that is adsorbed

1457 head-out.

1458 In a subsequent study $\mathrm{Li}$ and Gallus $[188,189]$ investigated C16TAB adsorption on two

1459 kaolinites, KGa-1b and KGa-2 (Clay Mineral Repository; Purdue University, West Lafayette,

1460 IN, USA); the CECs determined by an ammonia method were 30 and $37 \mathrm{mmol} / \mathrm{g}$,

1461 respectively. Relatively crude surfactant and counterion isotherms were measured, together

1462 with the accumulative cation desorption The discussion of the results is not always

1463 convincing, but the cation release as function of the surfactant adsorption provides clear

1464 information about the situation up to the CMC. Around the CMC the maximum amounts of

$1465{\mathrm{C} 16 T A^{+}}^{+}$adsorbed of about 58 and $85 \mu \mathrm{mol} / \mathrm{g}$ were reached and the corresponding amounts

1466 of cation released were about $18 \pm 2 \mu \mathrm{mol}_{\mathrm{e}} / \mathrm{g}$ and $11 \pm 2 \mu \mathrm{mol}_{\mathrm{e}} / \mathrm{g}$ for KGa-1b and KGa-2,

1467 respectively. However, at about $10 \mu \mathrm{mol} / \mathrm{g}$ surfactant adsorption the total cation release was

1468 already about $90 \%$ of the maximum release, and at 30-40 $\mu \mathrm{mol} / \mathrm{g}$ nearly all the cations were

1469 released. This indicates that the adsorption in the second layer started at adsorption values

1470 of about 10-15 $\mu \mathrm{mol} / \mathrm{g}$; upon further surfactant adsorption a small fraction of the ions will

1471 still be head-on adsorbed, but the majority of surfactant ions was adsorbed head-out (second

1472 layer). Therefore, the head-out adsorption started at surfactant concentrations clearly below

1473 the CMC and the contribution of the head-out adsorption (by hydrophobic attraction) was 
1474 far greater than that of the head-on adsorption (by cation exchange) and this means that

1475 asymmetric admicelles were formed. In the kinetic experiments the adsorption of bromide 1476 ions differed by about $15 \mu \mathrm{mol} / \mathrm{g}$ from the surfactant adsorption, which is close to the above 1477 estimate of the head-on adsorption. FTIR measurements showed a consistent shift of C-C 1478 symmetric and asymmetric vibration from high to low frequencies, which supported the 1479 view of a transition from head-on adsorbed monomers to admicelles as the C16TAB 1480 adsorption increased.

$1481 \mathrm{Li}$ and Gallus [189] also investigated the effect of surfactant chain length (C12TAB and 1482 C16TAB) and surfactant mixtures on kaolinite. As expected the surfactant chain length had no 1483 effect on the head-on adsorption (= cation exchange or first layer adsorption), but it did affect 1484 the adsorbed amount in the head-out mode (second layer). For C16TAB the head-out 1485 adsorption was considerably larger than the head-on adsorption, but for C12TAB the situation was reversed. For the 50/50 mixture the adsorbed amounts in head-on and head-out mode were comparable. Regardless of how much alkyl-ammonium adsorbed at the adsorption

1488 plateau, the amounts of cations desorbed were 15-18 $\mu \mathrm{mol}_{\mathrm{e}} / \mathrm{g}$ for KGa-1b and 9-11 $\mu \mathrm{mol}_{\mathrm{e}} / \mathrm{g}$ 1489 for KGa-2, respectively. Therefore, the CEC of KGa-1b and KGa-2 as determined by 1490 alkyl-ammonium adsorption/cation desorption will be $15-18 \mu \mathrm{mol}_{\mathrm{e}} / \mathrm{g}$, and 9-11 $\mu \mathrm{mol}_{\mathrm{e}} / \mathrm{g}$, 1491 respectively. These values are much less than the values determined by an ammonia method 1492 [190] or the reference values listed on Clay Mineralogical Society website 1493 (http://cms.lanl.gov/ chem.htm). However, the value of cations desorbed from KGa-1b 1494 through surfactant adsorption was similar to the CEC values of KGa-1 determined by a 1495 complexation method using copper bisethylenediamine and copper triethylenetetramine in the 1496 presence and absence of the buffer tris(hydroxymethyl)- aminomethane [191]. Comparing this 1497 with the results of Mehrian et al. [179] would imply that the difference should be related to 1498 the CEC of the plates as compared to the total CEC (plates + edges). 
1499 Recently, Malek and Ramli. [192] investigated C16PB on kaolinite with respect to 1500 antibacterial activity. Based on X-ray diffraction they first concluded that the structure of 1501 kaolinite was not affected by the surfactant adsorption. Although the interpretation of the

1502 results by the authors contains several errors, it is interesting that the antibacterial activity 1503 started after the IEP, i.e., when the surfactant started to adsorb in the head-out mode that 1504 made the overall particle charge positive, which must have caused the action of $\mathrm{C}_{16 \mathrm{P}^{+}}$ 1505 against the bacteria cells.

1506

\subsection{Anionic surfactant adsorption on kaolinite}

1508 Adsorption of anionic surfactants to kaolinite also occurs and for this adsorption the 1509 amphoteric edge sites are crucial. The point of zero net proton charge of the edge of 1510 kaolinite depends on the ionic strength and kaolinite composition, and is found to be in the $1511 \mathrm{pH}$ range 4 to 7 [193]. Therefore, the net proton charge of the edge is positive at relatively 1512 low $\mathrm{pH}$ and negative at relatively high $\mathrm{pH}$. The investigations of anion adsorption have 1513 mainly been carried out at relatively low $\mathrm{pH}$, which can lead to some dissolution of 1514 kaolinite and surfactant precipitation so that next to surfactant adsorption also surfactant 1515 (surface) precipitation occurs. The total amount of surfactant removed from the solution is 1516 therefore called the surfactant abstraction. Somasundaran et al. [168-170, 194, 195] have

1517 investigated these aspects in detail in their studies of sodium dodecyl benzenesulfonate and 1518 sodium dodecyl sulfonate abstraction by kaolinite. Results obtained under various ionic 1519 strengths and $\mathrm{pH}$ values indicated a complex abstraction mechanism involving surfactant adsorption by ion exchange and hydrophobic attraction and metal activated adsorption and surface precipitation of surfactant, all depending on the solution $\mathrm{pH}$. The precipitation of sulfonate with dissolved mineral species results in an adsorption maximum in micellar solutions. The dissolution of kaolinite and the resulting solution speciation can be found in

1524 [169]. Results for the abstraction of sodium dodecyl benzenesulfonate on Na-kaolinite 
1525 (Georgia kaolinite from the clay repository at University of Missouri subjected to an

1526 ion-exchange treatment) as a function of $\mathrm{pH}$ at $0.1 \mathrm{M} \mathrm{NaCl}$ and $25{ }^{\circ} \mathrm{C}$ as obtained by

1527 Siracusa and Somasundaran [195] are summarized in Fig. 22. The authors made the

1528 following remarks concerning the abstraction (adsorption) mechanisms. (1) At acidic pH

1529 levels below pH 3.7 significant precipitation of the dodecylbenzene sulfonate with cationic

1530 dissolved aluminum species occurs and subsequent re-dissolution of the

1531 aluminum-sulfonate precipitates above the CMC produces an abstraction maximum. (2) At

1532 or near $\mathrm{pH} 4.4$ (the pznpc of the sample) a reduction in the concentration of dissolved

1533 cationic aluminum species results in decreased precipitation such that the adsorption

1534 maximum is no longer observed. However, some contribution of aluminum-sulfonate

1535 precipitation is still present. (3) At $\mathrm{pH}$ levels above $\mathrm{pH} 4.4$, both in the neutral region $(\mathrm{pH}$

1536 7.9) and the alkaline region ( $\mathrm{pH}$ 10.8), incongruent dissolution of kaolinite can result in

1537 phase transformations to gibbsite; therefore, adsorption occurs at levels governed not only

1538 by the surface of the kaolinite, but also by that of gibbsite.

1539 Very similar $\mathrm{pH}$ dependent results have been obtained by Torn et al. [196] for the

1540 dodecylbenzenesulfonate / Na-Kaolinite (Sigma Company) system at $0.01 \mathrm{M} \mathrm{NaCl}$ and 25

$1541{ }^{\circ} \mathrm{C}$, but in this case the abstraction maximum occurred at $\mathrm{pH}$ 4.8-4.9. Proton titrations of the

1542 kaolinite sample suggested a pznpc of about 7 [197], which is higher than the pznpc of the

1543 kaolinite used by Siracusa and this might explain the shift of the maximum. Torn et al. [196]

1544 concluded that both electrostatic and hydrophobic interactions play a role in the adsorption

1545 of the sulfonate surfactant to kaolinite.

1546 Poirer and Cases [167] have studied the effect of chain length (C14 and C12) and the

1547 presence of a benzene ring in the apolar chain (C10Ф) of the sulfonate surfactants on their

1548 adsorption to kaolinite. The adsorption isotherms at $28{ }^{\circ} \mathrm{C}$ showed a sharp increase in

1549 abstraction close to the saturation concentration of the surfactants, which was due to the

1550 precipitation of the sulfonates. The isotherms were shifted toward lower equilibrium 
1551 surfactant concentrations as the hydrophobic moiety of the surfactant increased. The shifts

1552 were due to the increase in hydrophobic attraction and corresponded with the differences in

1553 CMC with surfactant structure. The benzene ring was about equivalent to three methylene

1554 groups. Del Hoyo et al. [166] investigated the adsorption of SDS on kaolinite by FTIR

1555 measurements and observed modification of the $\mathrm{CH}$ stretching wavenumbers of SDS

1556 indicating a re-arrangement of the adsorbed surfactant molecules and hydrophobic attraction

1557 between the hydrocarbon chains of the surfactant.

1558 How the structure of the adsorbed surfactant layer evolved as a function of the surfactant

1559 concentration has not been discussed in the above mentioned studies, but most likely also in

1560 this case head-on adsorption occurs at low adsorption values and head-out adsorption at

1561 surfactant concentrations closer to the CMC.

1562

1563

\subsection{Nonionic surfactant adsorption on kaolinite}

1564 The adsorption of nonionic surfactants on kaolinite resembles that on silica. Also on 1565 kaolinite the surfactant monomers adsorb by hydrogen bonding between the 1566 polyoxyethylene segments and the hydroxyl groups on the surface. In addition interaction 1567 may occur with the hydrated cations on the siloxane basal plate as is observed for 1568 montmorillonites [198]. The energy involved in the initial adsorption process is somewhat 1569 stronger than with silica. Similar as for silica, the energetic balance at the beginning of the

1570 adsorption is exothermic, because contacts between the surface and the ethoxy segments of 1571 the polar chains are enthalpically favorable. When the coverage of the surface increases, the 1572 enthalpy decreases down to the appearance of an endothermic regime. In the latter regime 1573 the molar enthalpy of adsorption is comparable to that of micellization in aqueous solution 1574 and this is characteristic for the presence of surfactant admicelles (or two adsorption layers) 1575 on the surface [161]. The temperature dependence of the adsorption [161, 199] is in 1576 agreement with this adsorption mechanism. Two steps can be observed along the adsorption 
1577 isotherms [144, 161, 199, 200], some typical isotherms are depicted in Fig. 23. The first

1578 pseudo-step occurs at low coverage and is followed by a rapid but gradual rise of the

1579 adsorption up to the CMC, somewhat above the CMC the adsorption plateau is reached.

1580 Due to the polydispersity of most nonionic surfactants the plateau starts somewhat beyond

1581 the CMC. The second step corresponds with a cooperative adsorption mechanism and the

1582 formation of admicelles. The relative importance of the first step increases with the EO

1583 chain length (stronger adsorption), but the adsorption level at the CMC strongly decreases

1584 with EO chain length because the large head group makes it more difficult for the apolar

1585 chains to associate by hydrophobic attraction. The plateau adsorption is reached at higher

1586 surfactant concentrations for longer EO chains. The reason for this is that when oxyethylene

1587 length rises, the monomer solubility in water is higher and, thus, the CMC is also higher.

1588 For different kaolinite samples (Supreme, Charentes, Aldrich, Sigma) the isotherms

1589 have a similar shape, but the adsorption levels of the first step and the final plateau around

1590 the CMC differ [200], which implies that the structural organization of the surface

1591 aggregates is dependent on the surface heterogeneity of the kaolin crystals. Based on the

1592 characteristics of the kaolinite samples and the adsorption results no final conclusion could

1593 be reached regarding the role of the edge surfaces and the basal surfaces.

1594

1595

\subsection{Anionic surfactant adsorption on montmorillonite}

1596 The adsorption of surfactants to montmorillonite differs from that of kaolinite for two

1597 reasons. (1) Kaolinite has two different basal plate surfaces and a relatively large edge

1598 surface, while the basal plate surfaces for montmorillonite are the same and the edge area is

1599 relatively small. (2) Montmorillonite is an expanding clay-type, which implies that the

1600 adsorption capacity may increase with increasing surfactant adsorption, depending on the

1601 conditions. Anionic surfactant adsorption will mainly occur on the edges and the anions will

1602 not easily intercalate between the clay platelets, this and the relatively small edge area of 
1603 montmorillonite make anionic surfactant adsorption on pure montmorillonite relatively

1604 unimportant. Similarly as for kaolinite, adsorption of anionic surfactants to montmorillonite

1605 is complicated by surfactant precipitation when multivalent ions are present as counterions

1606 of the negative clay charge and by partial clay dissolution and precipitation phenomena as

1607 described for kaolinite. For instance, Yang et al. [201] have shown that the abstraction

1608 (adsorption + precipitation) of sodium dodecylbenzene sulfonate (SDBS) by

1609 montmorillonite was affected by the presence of $\mathrm{Ca}^{2+}$ ions. They observed that: (i) SDBS

1610 was abstracted significantly by montmorillonite saturated with $\mathrm{Ca}^{2+}$, but little by

1611 Na-saturated montmorillonite; (ii) the amount of SDBS abstracted by Ca-montmorillonite

1612 was enhanced by $\mathrm{NaCl}$; and (iii) no significant intercalation of SDBS into

1613 Ca-montmorillonite was observed by X-ray diffraction analysis. Therefore the abstraction

1614 for the Ca-montmorillonite was largely due to surfactant precipitation with Ca released by

1615 ion exchange.

1616

1617

1618

1619

1620

1621

1622

1623

1624

1625

1626

1627

\subsection{Cationic surfactant adsorption on montmorillonite}

The binding of cationic surfactants on montmorillonite is dominated by adsorption at the plate surfaces, therefore, for these surfactants adsorption in the interlayers plays an important role. The adsorption behavior at the external surface of montmorillonite will be similar to that on silica and/or kaolinite, but the adsorbed amount and the conformation of adsorbed molecules in the interlayer space are affected by the two neighboring surfaces and the distance between these surfaces (the interlayer distance). In fact one deals with adsorption in a narrow slit pore with the complication that the slit width increases step wise with increasing surfactant adsorption. The interaction between the platelet surfaces and, therefore, the interlayer distance, are affected by the surfactant adsorption and vice versa. In other words, the interlayer distance, the adsorbed amount of surfactant and the conformation 
1628 of the adsorbed surfactant molecules are mutually related and this makes the adsorption

1629 behavior complicated.

1630 To unravel cationic surfactant adsorption in practice, next to information on the adsorbed 1631 amount, at least, also information is required on the interlayer distance and/or the 1632 conformation of the adsorbed surfactant molecules in the interlayer. Somewhat similar to 1633 Mehrian et al. [180], Pan et al. [202] measured C12TA+/C12TAB isotherms and adsorption 1634 enthalpies for $\mathrm{Na}$ - and $\mathrm{Al}$-montmorillonites in a wide $\mathrm{pH}$ range; the results support the 1635 two-stage adsorption above the IEP. Very illustrative experimental results on the adsorption 1636 of hexadecyl-trimethylammonium $\left({\mathrm{C} 16 \mathrm{TA}^{+}}^{+}=\right.$HDTMA) on montmorillonite (Wyoming 1637 montmorillonite, SWy-1; CEC $=900 \mu \mathrm{mol}_{\mathrm{e}} / \mathrm{g}$ ) and kaolinite (Na-kaolinite, CEC = 40 $1638 \mu \mathrm{mol}_{\mathrm{e}} / \mathrm{g}$ at $\mathrm{pH}$ 6.5) are provided by Xu and Boyd [184, 203]. They studied the adsorption by 1639 combining adsorption isotherms, electrophoretic mobility, colloidal stability and X-ray 1640 diffraction to determine the effects of clay type and solution composition on the adsorption 1641 of cationic surfactants and to unravel the orientation and/or conformation of the adsorbed 1642 surfactants on the external and internal (interlayer) surfaces and the dispersion stability of 1643 surfactant-clay complexes. Some of their results are depicted in Figs. 24 and 25. Fig. 24 1644 shows the isotherms of C16TAC on three different clays; isotherms provide information on 1645 the total surfactant adsorption. With a single isotherm further information is required to 1646 make a distinction between the adsorptions on external and internal surfaces. The C16TAC 1647 adsorption isotherm on Ca-montmorillonite (Ca-SWy-1) follows the 'normal' isotherm 1648 pattern and is similar to that on Na-kaolinite (non-swelling), but the adsorption isotherm on 1649 Na-montmorillonite (Na-SWy-1) differs from the other isotherms with its peculiar S-shape 1650 at relatively low C16TAC adsorption $(<1$ CEC). The fact that the shape of the C16TAC 1651 isotherms on Na-kaolinite and Ca-SWy-1 are rather similar indicates that the swelling of 1652 C16TA/Ca-SWy-1 due to surfactant adsorption is negligible small. The peculiar S-shape of 1653 the of C16TA/Na-SWy-1 system with different adsorption values for one equilibrium 
1654 concentration must be due to interlayer expansion due to surfactant adsorption. The

1655 behavior of the C16TA+/Na+-montmorillonite clay is further illustrated in Fig. 25 to 1656 provide proof for this behavior.

1657 The C16TA ${ }^{+}$/C16TAC isotherm on Na-SWy-1 (Fig. 25a) can be divided into four distinct 1658 regions. Region 1 (0-0.75 CEC) is non-monotonic and characterized by an equivalence of 1659 Na release and C16TA ${ }^{+}$adsorption, resulting in superimposable C16TA ${ }^{+}$adsorption and 1660 cation release curves. In regions 2 and 3, the adsorption isotherm is monotonic and the 1661 adsorbed amount of $\mathrm{C} 16 T A^{+} / \mathrm{C} 16 \mathrm{TAC}$ is greater than the corresponding $\mathrm{Na}^{+}$release; this 1662 implies that the head-on adsorption (ion exchange) slows down and the head-out adsorption 1663 increases (difference between the $\mathrm{C}_{16 \mathrm{TA}}{ }^{+} / \mathrm{C} 16 \mathrm{TAC}$ and $\mathrm{Na}^{+}$isotherms). The head-out 1664 adsorption is due to surfactant adsorption to already adsorbed surfactants by hydrophobic 1665 attraction between the surfactant tails. The slope of the C16TA ${ }^{+} /$C16TAC adsorption 1666 isotherm in region 3 is much larger than in region 2, which indicates cooperative adsorption

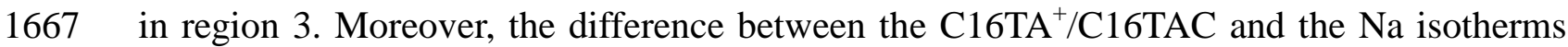
1668 increases strongly, indicating that head-out adsorption to already adsorbed surfactants 1669 increases much stronger than the ion exchange. At the CMC the C16TA ${ }^{+}$C16TAC and Na 1670 isotherms reach a plateau (region 4).

1671 Fig. 25b shows the behavior of the electrophoretic mobility with increasing surfactant 1672 adsorption; it reflects the situation at the external surface of the clay. In region $1 \mathrm{Na}^{+}$ 1673 exchanges for $\mathrm{C}_{16 \mathrm{TA}^{+}}$, therefore it is to be expected that the electrophoretic mobility of the 1674 C16TA/Na-SWy-1 particles remains the same as for homoionic Na-SWy-1; this is indeed 1675 the case. In region 2 (0.75 CEC to $1.0 \mathrm{CEC})$ the net particle charge is relatively low; more $1676 \mathrm{C} 6 \mathrm{TA}^{+}$is adsorbed than $\mathrm{Na}^{+}$is released and the electrophoretic mobility increases rapidly, 1677 it passes the IEP (particle charge changes sign) and reaches a maximum value at 1CEC, the 1678 end of region 2 . The fact that the IEP is reached before $\mathrm{C} 16 T A^{+}$(ads) $=$CEC implies that 1679 the $\mathrm{Na}+$ ions also contribute to the charge neutralization at the IEP. The fact that the 
1680 mobility changes sign confirms that the surfactant adsorption is super-equivalent and this

1681 implies that beyond the IEP head-out adsorption by hydrophobic attraction starts. The

1682 mobility is very sensitive to changes in charge around the IEP where the particle charge is

1683 low, therefore the information in region 2 is directly related to the charge situation of the

1684 clay complex and provides valuable information. In region 3 the mobility decreases slightly

1685 and stays constant in region 4. Somewhat outside the IEP (zeta potentials $>50 \mathrm{mV}$ )

1686 retardation and relaxation effects can easily lead to only small changes in mobility with

1687 particle charge [204]. Moreover, the changing particle (aggregate) sizes (see the stability)

1688 further complicate the situation. Therefore, further interpretation of the mobilities in regions

$1689 \quad 1$ and 3 or 4 is not feasible.

1690 The adsorption behavior at the external surface was further investigated by dispersion

1691 stability measurements (optical density of the clay suspension), see Fig. 25b. The degree of

1692 clay dispersion decreases with increasing surfactant adsorption till the IEP is reached. This

1693 is due to (i) the decrease of the net negative charge of the C16TA/Na-SWy-1 particles, so

1694 that the electrostatic repulsion between the particles decreases and (ii) at the same time the

1695 hydrophobicity of the particles increases because the surfactant tails point to the solution

1696 (ion exchange and head-on surfactant adsorption) and this increases the inter-particle

1697 (hydrophobic) attraction. The stability has a minimum around the IEP because the net

1698 particle charge is zero and the amount of unscreened surfactant tails pointing towards the

1699 solution is at its maximum, which makes the particles maximally hydrophobic. Beyond the

1700 IEP further C16TA ${ }^{+}$adsorption in the head-out mode makes the C16TA ${ }^{+}$-SWy-1 particles

1701 positive and increases the particle hydrophilicity, together this results in a strong increase in

1702 the dispersion stability.

1703 In Fig. 25c d(001)-spacings obtained by X-ray diffraction are shown for water saturated,

1704 partially wet (dried at 95\% relative humidity, $\mathrm{RH}$ ), and dry (dried at 5\% RH)

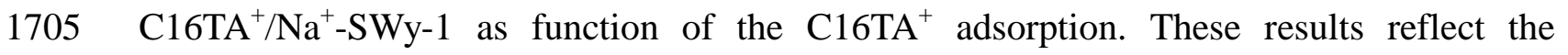


1706 situation at the interlayer surfaces. The clays are nearly identical at high $\mathrm{C}_{16 \mathrm{TA}^{+}}$adsorption,

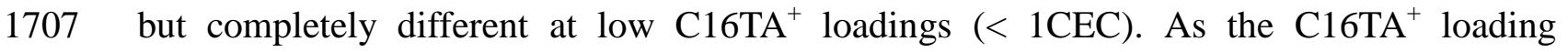

1708 increased from 0.1 CEC to 0.75 CEC (region 1), the d-spacings of wet (100\% RH) clay 1709 samples (C16TA ${ }^{+} / \mathrm{Na}^{+}$-SWy-1) increased gradually from 1.76 to $2.21 \mathrm{~nm}$. No further change

1710 in the d-spacing was observed at higher $\mathrm{C} 6 \mathrm{TA}^{+}$adsorption. Partial air-drying (95\% RH) or

1711 drying (5\% RH) of the clays decreases the d-spacings and results in stepwise d-spacing vs.

$1712 \mathrm{C}_{16 \mathrm{TA}}^{+}$loading curves. At loading levels up to $0.3 \mathrm{CEC}$, the d-spacing was around $1.4 \mathrm{~nm}$.

1713 The first step occurs between 0.3 and 0.5 CEC and raises the spacing to about $1.8 \mathrm{~nm}$, the

1714 second step occurs at $\mathrm{C}_{16 \mathrm{TA}^{+}}$loadings of 0.5 to $0.8 \mathrm{CEC}$ leads to a final d-spacing of about

$17152.2 \mathrm{~nm}$. The steps are sharp for the dry clay. The step-wise behavior of the d-spacing

1716 indicates that the conformation of the adsorbed surfactant ions changes stepwise. Confining

1717 the interlayer to lower values of the d-spacing by drying allows only specific surfactant

1718 conformations and sudden conformational changes result. However, at $100 \% \mathrm{RH}$ the

1719 d-spacing increases gradually, therefore the capacity for surfactant adsorption increases

1720 gradually and the actual conformation of the adsorbed surfactant is more random than for

1721 the (partially) dried clays.

1722 With the C16TA/Ca-SWy-1 system and adsorption values of about 0.25 CEC to 0.8 CEC

1723 the d-spacing stays around $1.8 \mathrm{~nm}$ and then increases gradually with higher loadings to 2.16

$1724 \mathrm{~nm}$ (not shown). The higher charge of the $\mathrm{Ca}^{2+}$ ions keeps the two interlayer surfaces better

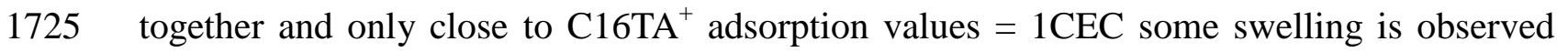

1726 and the isotherm has a similar shape as the $\mathrm{C} 16 \mathrm{TA}^{+}$isotherm to kaolinite. The peculiar

1727 shape of the C16TA ${ }^{+}$isotherm for Na-SWy-1 and the 'normal' shape of the isotherm for

1728 Ca-SWy-1 is thus due to the fact that in the adsorption range 0-0.8 CEC swelling occurs for

1729 Na-SWy-1 and no swelling for Ca-SWy-1. Due to swelling and a concomitant change of the

1730 adsorption capacity in the case of the C16TA/Na-SWy-1 system different adsorption values

1731 can occur at one equilibrium surfactant concentration. 
1732

1733

1734

1735

1736

1737

1738

1739

1740

1741

1742

1743

1744

1745

1746

1747

1748

1749

1750

1751

1752

1753

1754

1755

1756

1757

When for the C16TA/Na-SWy-1 system the ionic strength is increased by increasing the $\mathrm{NaCl}$ concentration, the isotherms gradually change to the 'normal' shape at about 42 $\mathrm{mmol} / \mathrm{L} \mathrm{NaCl}$. At this ionic strength the d-spacing stays about constant up to $0.8 \mathrm{CEC}$. Around loadings close to the $\mathrm{CEC}$ the isotherms at different $\mathrm{NaCl}$ concentration intersect and after the intersection point (loadings larger than the CEC) the adsorption increases with increasing $\mathrm{NaCl}$ concentration. The residual water in the interlayer allows salt ions to enter the interlayer, and similarly as at the external surface, this leads to an increased screening of the lateral repulsion between the charged head-groups of super-equivalently bound surfactant ions and an increase in adsorption. For the same reason also surfactant counterions that partially associate with the surfactant ions increase the surfactant adsorption for loadings beyond the CEC. The $\mathrm{NaCl}$ concentration has little effect on the C16TA/Ca-SWy-1 system at C16TA ${ }^{+}$loadings up to the CEC and after the CEC the behavior is similar to than of the C16TA/Na-SWy-1 system.

Concluding, based on the results of $\mathrm{Xu}$ and Boyd [184] it follows that the swelling of expanding clays due to cationic surfactant adsorption depends on the strength at which the clay layers are held together. It is well known that the attraction between the clay layers depends on the degree of isomorphic substitution, the valence and size of the counterions of the clay charge and the ionic strength [205]. Therefore, also the swelling of clays due to cationic surfactant adsorption is depending on these variables. Swelling occurs when the surfactant isotherm shows a peculiar S-shape for surfactant loadings up to 0.8 CEC; the adsorption capacity is a function of the adsorbed amount and this makes it possible that different adsorption values may occur at one equilibrium surfactant concentration. The surfactant adsorption at the external surface of expanding clays shows a very similar behavior to that at kaolinite. Up to loadings close to the IEP of the external particle surface (roughly corresponding with a loading approaching 1 CEC) the surfactant adsorption occurs by ion exchange and head-on adsorption. Beyond the IEP of the external surface (or 
1758 loadings $\geq 1 \mathrm{CEC}$ ), adsorption of the surfactant by hydrophobic attraction starts and this

1759 adsorption increases strongly with further surfactant loading. The adsorption beyond the 1

1760 CEC increases with increasing ionic strength and is sensitive to the kind of surfactant

1761 counterion. The clay stability is strongly affected by this adsorption behavior. The minimum

1762 dispersion (colloidal) stability occurs at the IEP of the external surface where the net

1763 particle charge is zero and the hydrophobicity of the particles is at its maximum due to the

1764 unscreened surfactant tails protruding into the solution. The results of $\mathrm{Xu}$ and Boyd are

1765 largely supported by Tahani et al. [206] who investigated the adsorption of the

1766 benzyldimethyl dodecyl ammonium chloride on a Na-montmorillonite by various

1767 techniques.

1768 An interesting complementary study, specifically oriented towards the structural 1769 characteristics of the surfactant adsorbed in clay interlayers, has been presented by Zhu et al.

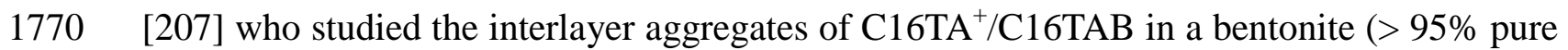
1771 montmorillonite, < 5\% quartz; CEC $1084 \mu \mathrm{mol}_{\mathrm{e}} \mathrm{g}$ ) for different surfactant loading levels 1772 (0.20 CEC to 2.56 CEC), using in situ X-ray diffraction (XRD) and Fourier Transform Infra 1773 Red (FTIR) spectroscopy on samples in the hydrated state. For the dried and hydrated 1774 organo-bentonites, the measured basal spacings depended on surfactant loadings and 1775 indicated that the water molecules present play an important role in regulating the 1776 microenvironment, even at high surfactant loads. In the presence of water the surfactant 1777 tails formed aggregate structures in the interlayer space due to hydrophobic attraction. As a 1778 result, the values of the d001 spacing increased gradually with increase in surfactant loading. 1779 In situ FTIR spectra indicated that the surfactant intercalated at high loading had a more 1780 ordered and compact structure than the associates at low surfactant sorption density: the 1781 surfactant state in wet organic bentonites changed from a liquid-like (disordered) state to a 1782 solid-like (ordered) state. 
Based on the FTIR results, the XRD data, and the dimensions of the C16TAB surfactant

1784 in moist organo-bentonites with different surfactant loading, Zhu et al. proposed that

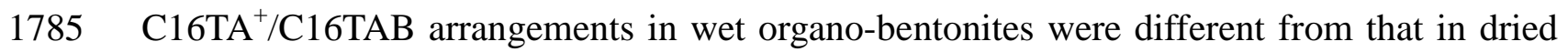

1786 organo-bentonites. For the wet organo-bentonites the interlayer spacing around 0.2 CEC is

$17871.02 \mathrm{~nm}$ and the $\mathrm{C}_{16 \mathrm{TA}^{+}}$ions lie about parallel to the silicate interlayer surfaces. Then a

1788 smooth step in the interlayer spacing occurs and at 0.4 to 1.2 CEC the spacings of $2.0-2.5$

$1789 \mathrm{~nm}$ are roughly the length of $\mathrm{C} 6 \mathrm{TA}^{+}$. Therefore, a possible arrangement could involve the

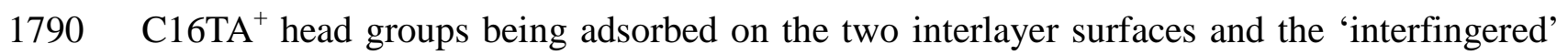

1791 long alkyl chains roughly perpendicular to the interlayer surfaces. When the loading level

1792 further increases (1.2 to 2.6 CEC), a next smooth step in interlayer spacing occurs to about

$1793 \quad 3.3 \mathrm{~nm}$ and the C16TA ${ }^{+}$ions (ion exchanged) and C16TAB molecules (hydrophobically

1794 attracted) form a partly 'interfingered' admicellar type layer. With these surfactant

1795 conformations at loadings $>0.4$ CEC the surfactant phase in the interlayer is divided into

1796 three regions: region 1 and 3 are adjacent to the two interlayer surfaces, each extending to

1797 about $0.5 \mathrm{~nm}$ from the surface, containing $\mathrm{H}_{2} \mathrm{O}$, surfactant head groups and, if loaded in

1798 excess of the CEC, also the surfactant counterions (Br-), and region 2 with a thickness of

1799 about $2 \mathrm{~nm}$ in the center of the interlayer space, containing the aggregated tails of the alkyl

1800 chains and residual water. The total structure is bilayer-like and/or resembles flattened

1801 admicelles. The core region with the tails becomes more aliphatic and less aqueous as the

1802 surfactant loading increases from 0.40 to 2.6 CEC. With increasing surfactant loading the

1803 core region becomes more hydrophobic, but induced steric hindrances increase and the

1804 space left for ad-solubilization decreases; therefore, the partitioning of organic contaminants

1805 shows a maximum around loading levels of 1 CEC. This maximum corresponds roughly

1806 with the maximum hydrophobicity of the surfactant layer at the external surfaces of the clay

1807 around loadings of 1 CEC, but the structure of the external surfactant layer is rather 
1808 different (head-on monolayer type and/or teepee-like micelles) from that in the interlayer

1809 (flattened admicelle-like).

1810 The specific behavior of surfactant adsorption in confined space has also been 1811 investigated theoretically by Molecular Dynamics studies. Initially these studies modeled 1812 dry organo-clays [177, 208-210] but in later studies water was included in the simulations

1813 [211, 212]. The latter are most relevant with respect to understanding the adsorbed 1814 surfactant conformations in the interlayer space. The introduction of the paper by Liu et al. 1815 [178] provides a brief overview of the history of MD calculations on organo-clays and the 1816 force fields used; in that of Zhou et al. [213] the most relevant experimental results and the 1817 recent MD simulations are mentioned. Liu et al. [178] discuss the structure and dynamics of 1818 alkylammonium-intercalated wet SWy-type montmorillonite with emphasis to the alkyl 1819 chain length; the paper is complementary to the recent paper of Zhou et al. [213], which will 1820 be discussed here. The study of Zhou et al. [213] on C16TA ${ }^{+} / \mathrm{C} 16 \mathrm{TAB}$ intercalated 1821 Ca-montmorillonites (Mt) in the water-saturated condition largely mimics the experimental 1822 system of Zhu et al. [207] discussed above, and provides a good illustration of the 1823 information that can be gained by MD simulations.

1824 The simulation results of Zhou et al. [213] show that, as the surfactant adsorption on 1825 montmorillonite (CEC $1060 \mu \mathrm{mol} / \mathrm{g}$ ) increases, the arrangement of $\mathrm{C}^{26 \mathrm{TA}^{+} / \mathrm{C} 16 \mathrm{TAB}}$ 1826 transforms from flat bilayer-type to inclined paraffin-type with a large amount of water in 1827 the interlayer space. This configuration is different from that in dry systems; for dry samples 1828 the surfactant conformation changes from monolayer to bilayer and to pseudo-trilayer as 1829 surfactant loading increases $[171,175]$. Compared to the dry models, the percentage of 1830 gauche conformations of $\mathrm{C} 16 \mathrm{TA}^{+}$decreases in the water saturated condition. The head 1831 groups of $\mathrm{C} 16 \mathrm{TA}^{+}$are located close to the centers of the six-member rings of the silicate 1832 surfaces and coordinated with 4-6 water molecules. Their mobility is low due to the 1833 electrostatic interactions, while the alkyl chains show a higher mobility. Montmorillonite 
1834 with a high CEC $(1310 \mu \mathrm{mol} / \mathrm{g})$ has a stronger confining effect on both $\mathrm{C} 16 \mathrm{TA}{ }^{+} / \mathrm{C} 16 \mathrm{TAB}$

1835 and water, which reduces the mobility of alkyl chains and water molecules within the 1836 interlayer space.

1837 A general impression of the surfactant conformations in the interlayer space as function 1838 of surfactant loading is provided by the density distributions for ammonium N, alkyl C, $1839 \mathrm{Ca}^{2+}, \mathrm{Br}^{-}$and water $\mathrm{O}$ [213]. The calculated density distributions for montmorillonite with a 1840 CEC of $1060 \mu \mathrm{mol}_{\mathrm{e}} / \mathrm{g}$ are depicted in Fig. 26 for different loading levels relative to the CEC 1841 as indicated in the figure. Corresponding snapshots of $\mathrm{C} 6 \mathrm{TA}^{+}$intercalated in the interlayer 1842 that further visualize the distribution are presented in Fig. 27. For Mt-0.25CEC-1nm (= 1843 montmorillonite with a C16TA+/C16TAB loading equal to $0.25 \mathrm{CEC}$ and an interlayer 1844 spacing of about $1 \mathrm{~nm}$ ) both ammonium $\mathrm{N}$ and alkyl $\mathrm{C}$ are arranged in two layers according 1845 to the two clear peaks of the density distributions, indicating a bilayer structure, see Figs. 1846 26a and 27a. As the surfactant loading level increases (Mt-0.5CEC-2nm; Figs. 26b and 27b), 1847 the density distribution of ammonium $\mathrm{N}$ remains two peaks close to the silicate surface due 1848 to the strong electrostatic attraction between the negative charge site and the cationic 1849 ammonium N. The distribution of alkyl C is rather different from that at 0.25 CEC, it 1850 transforms to one broad peak in the center and the arrangement of alkyl chains is inclined to 1851 the Mt surface. In these two incomplete cation exchange cases $\mathrm{Ca}^{2+}$ displays a symmetrical 1852 distribution with ammonium $\mathrm{N}$ to balance the residual negative charge. $\mathrm{Ca}^{2+}$ plays no role 1853 for surfactant loadings $>1.0$ CEC, but in this case the negative surfactant counterion $\left(\mathrm{Br}^{-}\right)$ 1854 comes into play. For Mt-1.0CEC-2.2nm (Figs. 26c and 27c), the situation is similar as for 1855 Mt-0.5CEC-2nm, but more pronounced. The broad central peak of alkyl is larger, indicating strong association of the alkyl chains in the interlayer space; the arrangement of alkyl chains 1857 is still inclined to the Mt surface. In the excessive surfactant loading case (Mt-2.0CEC-3nm; 1858 Figs. 26d and 27d), most $\mathrm{N}$ is still close to the surfaces, but part of $\mathrm{N}$ is distributed over the 1859 core of the interlayer. The $\mathrm{N}$ distribution is roughly paralleled by the $\mathrm{Br}$ distribution, but 
1860 close to the surface $\mathrm{N}$ and $\mathrm{Br}$ occupy separate layers. The magnitudes of the $\mathrm{N}$ and $\mathrm{Br}$ peaks

1861 close to the surface indicate that a large part of head groups that did not participate in the

1862 ion exchange is also located close to the surface. The small peaks of ammonium $\mathrm{N}$ in the

1863 interlayer space are balanced with $\mathrm{Br}$ in the core of the interlayer space. The alkyl chains are

1864 connected closely, reflecting the association by hydrophobic attraction. In all cases, the

1865 density of water near the Mt surfaces is somewhat higher than in the center, which is

1866 probably related to the hydration of the accumulated ions and ammonium head groups close

1867 to the surface. The hydrophilicity of the interlayer surfaces is somewhat questionable,

1868 because a siloxane surface without charge is fairly hydrophobic. The density distributions of

1869 interlayer species for Mt with a high CEC $(1310 \mu \mathrm{mol} / \mathrm{g})$ are in most cases similar as those

1870 shown here. However, for the Mt-0.25CEC-1nm system, the alkyl C forms several peaks

1871 near the center of the interlayer space instead of two peaks. The ammonium head groups

1872 close to the surface cause most likely obstruction of the orientation of the alkyl chains

1873 parallel to the surface. To sum up, under the water saturated condition and low surfactant

1874 loading the surfactant alkyl chains are oriented roughly parallel to the surface; at loadings $\geq$

18750.5 CEC alkyl chains are concentrated in the center of the Mt interlayer space and most

1876 head groups are close to the surface. The calculated results are consistent with the

1877 experimental results of Zhu et al. [207] and the density profiles are subtler versions of the

1878 schematic models suggested by Zhu et al.

1879 An alternative way of modeling surfactant binding from aqueous solutions in confined 1880 space is by Self-Consistent Field calculations with the SCFA model [214-217]. With the 1881 SCFA model surfactant isotherms can be calculated for two surfaces at a given distance and 1882 the interlayer spacing can only be changed by discrete step of about the diameter of a water 1883 molecule; simultaneous with the adsorption isotherm the interaction force between the two 1884 surfaces can be calculated. Calculations made [214-217] have not addressed the specific 1885 clay-surfactant systems, but some knowledge of the obtained results is still useful for a 
1886 better understanding of the complications that occur with surfactant adsorption in confined

1887 space. For charged hydrophilic surfaces the SCFA calculations have provided insight in the

1888 adsorption behavior below and above the charge compensation point [214, 215]. The

1889 confined space drives the adsorbed surfactant layer toward a structure similar to that

1890 obtained at the charge compensation point. At solid-solid separations where the surfactant

1891 layers on each surface begin to merge with each other (proximal adsorption), there is a

1892 confinement-induced phase transition leading to complex adsorption and interaction

1893 behavior at very small separations. In addition, calculations show that the interaction

1894 between surfactant tails is a key contributor to both the magnitude of proximal adsorption

1895 and the exchange between surfactant and co-ions at the surface. For (charged) hydrophobic

1896 surfaces [216, 217] a first-order phase transition takes place when the slit width approaches

1897 the thickness of the two surfactant layers; the transition is driven by the unfavorable

1898 hydrophobic-water contacts. At the transition, the average orientation of the surfactants

1899 switches from a high concentration of tails at the surface and head groups in the center to a

1900 bilayer configuration where the tail profiles from both sides merge in the center and the

1901 head groups are in the vicinity but slightly away from the surface. For more information the

1902 original papers should be consulted.

1903

19046.7 Nonionic surfactant adsorption on montmorillonite

1905 Similarly as with cationic surfactants the binding of nonionic surfactants on

1906 montmorillonite is dominated by adsorption at the plate surfaces, therefore, also for these

1907 surfactants adsorption in the interlayers plays an important role. Detailed results on the

1908 adsorption of nonionic surfactants on montmorillonite at low surfactant concentrations are

1909 scarce; recent results mainly concentrate on concentrations around and above the CMC

1910 because the interest is on preparation of organo-clays made with nonionic surfactants.

1911 Rheinländer et al. [218] have investigated C12(EO)8 on four different clays 
1912 (Na-montmorillonite $=$ Na-Mt, Ca-bentonite $(95 \%$ montmorillonite $)=$ Ca-Mt, Na-kaolinite

1913 and Na-illite) based on adsorption isotherms and for the swelling clay minerals (Na-Mt and

1914 Ca-Mt) microcalorimetry and X-ray diffraction. The measured isotherms were presented as

1915 double logarithmic plots that are reproduced in Fig. 28. The isotherm on Na-kaolinite runs

1916 below that on Na-illite, and both isotherms (mmol/g) are clearly below those on the swelling

1917 minerals. However, when the adsorbed amount is expressed per $\mathrm{m}^{2}$ the maximum adsorption

1918 values on the Na-clays are similar and about twice as high as that on Ca-Mt. The isotherms

1919 for Na-Mt and Ca-Mt are similar; however, the adsorption for Ca-Mt is stronger at low

1920 concentrations than that of $\mathrm{Na}-\mathrm{Mt}$, but at high concentrations the situation is reversed

1921 (maximum adsorptions Na-Mt and Ca-Mt, respectively, 0.94 and $0.35 \mathrm{mmol} / \mathrm{g}$ ). A greater

1922 (exothermic) initial differential molar enthalpy (heat) of adsorption confirms the higher

1923 affinity of C12(EO)8 for Ca-Mt than for Na-Mt. The heat of adsorption of the two clays

1924 decreases about linearly with surfactant loading and becomes zero above $0.26 \mathrm{mmol} / \mathrm{g}$, which

1925 is below the adsorption maximum of the two clays. The behavior of the heat of adsorption

1926 indicates two steps in the adsorption process: (i) adsorption due to interaction with the surface

1927 and (ii) adsorption by mainly hydrophobic attraction with already adsorbed surfactant. The

1928 observations that zero heat of adsorption to Ca-Mt and Na-Mt is reached at the same loading

1929 (mmol/g), but that the maximum amounts adsorbed are rather different indicates that the

1930 second adsorption step is larger for Na-Mt than for Ca-Mt. Although the adsorption process

1931 contains two steps, the shape of the log-log isotherms indicates that there is no critical surface

1932 association concentration (CSAC), all isotherms show a gradual increase in adsorption and

1933 the slope is of the isotherms is always $\leq 1$. Therefore, the transition of the first to the second

1934 adsorption process is gradual. This is the case for all four clay samples studied.

1935 The X-ray diffraction results showed that the basal (d001) spacing of pure air-dried

1936 Na-Mt of about $1.25 \mathrm{~nm}$ differed significantly from that of Ca-Mt of $1.5 \mathrm{~nm}$ due to stronger

1937 hydration of the $\mathrm{Ca}^{2+}$ than of $\mathrm{Na}^{+}$. The basal spacing of Na-Mt with surfactant goes from 
$19381.25 \mathrm{~nm}$ to $1.4 \mathrm{~nm}$ for $0.1-0.35 \mathrm{mmol} / \mathrm{g}$ and to about $1.8 \mathrm{~nm}$ for higher adsorption values.

1939 For Ca-Mt the spacing changes from 1.5 to $1.8 \mathrm{~nm}$ between 0.2 and $0.3 \mathrm{mmol} / \mathrm{g}$. These 1940 values are for both swelling clay minerals indicative of the formation of flat-lying 1941 C12(EO)8 molecules in the first adsorption step and of the formation of a 'flat' bilayer 1942 structure in the second adsorption step. As the spacings are derived from air-dried samples, 1943 the distances will be likely different in the water saturated situation (and therefore the 1944 surfactant conformation), but the two steps in the adsorption process are reflected in the 1945 measured values. The stronger hydration of $\mathrm{Ca}^{2+}$ than of $\mathrm{Na}^{+}$has, most likely, also caused 1946 the higher affinity of C12(EO)8 for Ca-Mt than for Na-Mt.

1947 Sonon and Thompson [219] investigated the adsorption of C12(EO) $<23>$ (=Brij 35; the 1948 notation $<$ n $>$ indicates that the EO chain length is polydisperse) on two smectites (Wyoming 1949 montmorillonite and Panther Creek smectite) that were brought in the K and Ca form. For 1950 both smectites the adsorption affinity and adsorption plateau were somewhat larger for the Ca-smectite than the K-smectites. The d001 values of both air-dried K-saturated smectites increased in two steps from $1.11 \mathrm{~nm}$ to about $1.7 \mathrm{~nm}$ as more surfactant was sorbed, while 1953 the d001 spacing of the air-dried Ca-smectites increased gradually from $1.47 \mathrm{~nm}$ to about $19541.7 \mathrm{~nm}$. These results confirm those of Rheinländer et al. [218].

1955 Backhaus et al. [220] continued the work of Rheinländer and investigated the adsorption of 1956 the octylphenol poly(ethylene oxide)s [(C8Ф(EO)<n>], TX100 $(<\mathrm{n}>=9.5)$, TX-165 $(<\mathrm{n}>=$ 1957 16) and TX-305 $(<\mathrm{n}>=30)$, on Ca-Mt and silica. Note that the hydrophobic character of a 1958 C8Ф group is very similar to that of a C12 group. The adsorption isotherms of $1959 \mathrm{C} 8 \Phi(\mathrm{EO})<9.5>(=\mathrm{TX} 100)$ on $\mathrm{Ca}-\mathrm{Mt}$ at $\mathrm{pH} 4$ and $\mathrm{pH} 9$ in $10^{-2} \mathrm{~mol} / \mathrm{L} \mathrm{CaCl}_{2}$ showed only 1960 small differences, which indicated that the share of the edge surfaces to the surfactant 1961 adsorption on Ca-Mt is small. Furthermore, the affinity (measured by the initial slope of the 1962 isotherm) of $\mathrm{C} 8 \Phi(\mathrm{EO})<9.5>$ at $\mathrm{pH} 4$ to Ca-Mt was higher than that on silica, which point to a 1963 different binding mechanism. With silica the EO segments mainly bind to the silanol groups 
1964 by H-bonds, but with expanding layered silicates these groups are hardly present and the

1965 binding mechanism is most likely H-bonds with the hydration water of the bound counterions on the clay surfaces. The interlayer spacings of Ca-Mt with adsorbed C8 $(\mathrm{EO})<9.5>$ in the dried and moist state show a similar behavior as in the case of C12(EO)8 [218], but the spacings for the moist sample are even at the largest adsorption values considerably (2nm) larger than the spacings of the air-dried sample. This indicates again that in aqueous solution 1970 the interlayer spacings will be different from that of the air-dried samples.

1971 The isotherms (non-logarithmic) of the three surfactants (TX100, TX-165, TX-305) [220] 1972 were all L2-type [221], i.e. a steep rise towards the maximum adsorption. For TX-100 1973 adsorption on montmorillonite or bentonite the L2 type isotherm has also been observed by 1974 [218, 222, 223]. Somewhat above the CMC the isotherms show a plateau that decreases with 1975 increasing EO head group length. The fact that the plateau adsorption is reached beyond the 1976 CMC is due to the fact that head group polydispersity leads to a relatively low CMC and a 1977 maximum adsorption at relatively high concentration [224]. The lack of the S-shaped course 1978 and thus of cooperative effects suggests that, unlike in the case of silica gel, no aggregate 1979 formation took place on the surface at low surfactant concentrations. This behavior is to be 1980 expected when the affinity of the surfactant molecules to the surface (hydrated Ca ions) is 1981 greater than the hydrophobic attraction between the surfactant molecules. With the relatively 1982 small interlayer spacing, the second step caused by hydrophobic attraction will be largely 1983 lateral (tail-tail) attraction. For steric reasons the lateral hydrophobic attraction decreases by 1984 the head group size and this explains the decreasing plateau adsorption with increasing head 1985 group size.

1986 A decrease in the adsorption with increasing head group size for a given alkyl chain length 1987 has also been observed by Shen [225] who measured the adsorption of C9(EO)<10>, $1988 \mathrm{C} 9(\mathrm{EO})<20>$ and $\mathrm{C} 18(\mathrm{EO})<20>(=$ Brij 78$)$ on bentonite at $\mathrm{pH} 6-7$ and $0.01 \mathrm{M} \mathrm{NaCl}$. 1989 Doubling the alkyl chain length at a given degree of ethoxylation increased the adsorption 
1990 maximum also but the effect was much smaller than that of doubling the head group size, 1991 especially when the adsorption was expressed as mmol/g. Deng et al. [226] who studied the 1992 adsorption of $\mathrm{C} 16(\mathrm{EO})<10>(=$ Brij-56) and $\mathrm{C} 9 \Phi(\mathrm{EO})<12>(=$ Igepal-CO720 $)$ on 1993 Ca-bentonite found somewhat larger adsorption for the surfactant with the smaller head 1994 group in line with the results of Shen [225]. However, the proposed surfactant conformation 1995 for the freeze-dried composites with EO segments in the interlayer and the alkyl chains 1996 sticking-out in air, is highly unlikely for water saturated samples and for the situation of the 1997 intercalated smectite in contact with an aqueous surfactant solution, because in this case 1998 hydrophobic attraction comes into play and the proposed conformation would lead to many 1999 unfavorable $\mathrm{CH}_{2}$-water contacts which can be avoided when the alkyl chains associate in 2000 the interlayer space. Therefore, it is to be expected that with adsorption from solution the 2001 conformation will be somewhat similar to that of cationic surfactants in the interlayer as described in the previous section.

2003

To unravel the EO binding mechanism and the EO chain conformation of intercalated 2004 polymeric nonionic surfactants Deng et al. [198] also investigated the intercalation of bentonites (with different cations) by: C16(EO)<10> (= Brij 56), C18(EO)<100> (= Brij 700), 2006 $\mathrm{C} 2(\mathrm{CH} 2-\mathrm{CH} 2)<15>(\mathrm{EO})<40>\quad$ ( $)$ polyethylene-polyethylene oxide or $\mathrm{PE}-\mathrm{PEO})$ and (EO) $<76>$ (= PEO). The surfactants and the organo-bentonites were characterized with X-ray 2008 diffraction (XRD) and Fourier transform infrared (FT-IR) spectroscopy. The surfactants 2009 intercalated the bentonite and expanded the d001 spacing of the freeze-dried samples to nearly $20101.8 \mathrm{~nm}$. The shapes and positions of the IR bands of interlayer surfactants resembled 2011 amorphous PEO. The EO segments of the surfactants were arranged in a distorted and extended form in the interlayer, instead of in the favored helical conformation of the crystalline state. The shifts of the $\mathrm{C}-\mathrm{O}-\mathrm{C}$ stretching bands of the intercalated surfactants 2014 pointed to H-bonding of the EO oxygen atom with water in the hydration shell of the 2015 exchangeable cations, or to direct coordination or ion-dipole interaction between the 
2016 exchangeable cations and the oxygen atoms. The type of exchangeable cations $\left(\mathrm{Na}^{+}, \mathrm{K}^{+}\right.$, $2017 \mathrm{Ca}^{2+}, \mathrm{Mg}^{2+}, \mathrm{Cu}^{2+}, \mathrm{Ni}^{2+}, \mathrm{H}^{+}$) in the interlayer of smectite did not affect the conformation of 2018 the EO chain of the intercalated surfactants. These results give valuable information on the 2019 binding mechanisms and indicate clearly that the (EO) $<$ n $>$ conformations in the interlayer of 2020 the freeze-dried samples are more disordered than in the crystalline state. However, as 2021 indicated above, in the case of water-saturated samples the conformation of the EO chains is 2022 likely more random and affected by the presence of the apolar segments in the interlayer, 2023 because the apolar segments will locally associate due to hydrophobic attraction and not 2024 protrude into the aqueous solution.

2025 The studies by Guégan [227, 228] on the intercalation of C10(EO)3 in the interlayers of 2026 Ca- and Na-montmorillonite (SWy-2) also deserve some attention because this surfactant 2027 easily forms in solution somewhat above the CMC a lamellar phase that consists of the 2028 stacking of molecules by hydrophobic attraction and it is well known that relatively small $2029 \mathrm{Cn}(\mathrm{EO}) \mathrm{m}$ surfactants with $\mathrm{m}<\mathrm{n}$, such as C12(EO)6, adsorb on silica with a condensation 2030 step just before the CMC in a bilayer arrangement [153, 164]. With Ca-Mt the adsorption 2031 isotherm of C10(EO)8 was high affinity with a pseudo plateau reached at about the CMC. 2032 X-ray diffraction and FT-IR measurements on samples dried at $70{ }^{\circ} \mathrm{C}$ indicated an 2033 expansion of the basal spacing to $1.7 \mathrm{~nm}$ and adsorption in two adsorbed monolayers 2034 parallel to the clay surface [227]. In a subsequent study [228] intercalation of Na-Mt by 2035 C10(EO)3 was investigated, the Na-Mt suspension was well swollen and showed a stable 2036 hydration state in which silicate layers were widely expanded and already opened when the 2037 surfactant solution was added, thus allowing an easier adsorption. Moreover initial solution concentrations of C10(EO)3 were selected that where several times the CMC which already 2039 displayed the L $\alpha$-lamellar phase. The adsorption values that where reached were similar and 2040 somewhat higher (40\%) than those in the Ca-Mt system. The results obtained with small 2041 angle X-ray scattering and with FT-IR (samples dried at $70{ }^{\circ} \mathrm{C}$ ) however revealed d001 
2042

2043

2044

2045

2046

2047

2048

2049

2050

2051

2052

2053

2054

2055

2056

2057

2058

2059

2060

2061

2062

2063

2064

2065

2066

spacings from $1.1 \mathrm{~nm}$ for Na-Mt and $3.8 \mathrm{~nm}$ for the loaded samples and in the interlayer space a well-ordered surfactant bilayer, with a structure similar to that of the lamellar phase. The X-ray diffraction patterns further indicated that the C10E3 molecules were not aggregated on the external clay surfaces. Although the translation of these results to the surfactant conformations that are adopted with adsorption from solution is not straight forward, it seems plausible that a kind of bilayer with a hydrophobic core in the interlayer space might also be possible with simple C10(EO)3 adsorption. Calculations with the SCFA model might provide further insight; calculations for nonionic surfaces have been made before $[152,153,165]$ and can be extended to adsorption in a confined space as has been done for ionic surfactants [214-217].

\section{Surfactant adsorption modeling}

\subsection{General remarks}

Surfactant adsorption isotherms have been qualitatively and (semi-) quantitatively interpreted using several adsorption models, ranging from the Langmuir equation to very sophisticated models. For a review of the models used to describe surfactant adsorption, including those specifically designed for surfactant adsorption to solid-liquid interfaces, the reader is referred to [25], where also references can be found. Here some classical models are briefly discussed in relation to surfactant adsorption. In general two types of modeling can be distinguished, (i) mean-field models in which the adsorbed layer is assumed to be smeared-out homogeneously over the surface and (ii) aggregation models in which the surfactants are adsorbed as aggregated, micellar type structures. Simple examples of the first category are the Langmuir-, the Frumkin-Fowler-Guggenheim- and the Freundlich -equation;. examples of the aggregate models are the Hill- and the Gu-equation. 
The famous Langmuir model applies to sorption from an ideally dilute solution of non-interacting molecules on homogeneous binding sites. The binding sites should be attached to particles, but the geometry of the particles and precise location of the sites is not relevant, only the homogeneity of the sites is important. The Langmuir (L) Eq. can be written as:

$$
\frac{\theta}{1-\theta}=K c
$$

2074

where $\theta$ is the fraction of the sites covered with the adsorbate, $c$ the equilibrium concentration and $K$ the equilibrium affinity constant accounting for all types of interactions with the sites. Preferably, the concentration, $c$, is expressed as normalized concentration, i.e., with respect to a chosen standard state: $c=c^{\prime} / c^{*}$, therefore, as both $c^{\prime}$ and $c^{*}$ should have the same units, $c$ is dimensionless. When the concentration $c^{\prime}$ is expressed in $\mathrm{mol} / \mathrm{L}$ a convenient standard state is $c^{*}=1 \mathrm{~mol} / \mathrm{L}$. For a good understanding of the obtained parameter values the standard state should be indicated. In the case of a normalized concentration also the affinity constant $K$ is dimensionless and can be expressed as a Boltzmann factor containing the standard molar Gibbs energy of adsorption: $K=$ $\exp \left(-\Delta G^{0} / R T\right)$, the surface covered with water (considered as a continuum) is taken as the standard state for the energy difference, $R$ is the universal gas constant and $T$ the absolute temperature. The site coverage can be expressed as the covered amount of sites divided by the total amount of sites, or, as is done most often in practice, the adsorption, $\Gamma$, divided by the maximum adsorption, $\Gamma_{\mathrm{m}}$, and in that case the L-equation becomes

$$
\Gamma=\frac{\Gamma_{m} K c}{1+K c}
$$

As the L-eq. applies to ideal sorption it will, in general, not be able to describe surfactant adsorption, but when it does, the obtained parameters $K$ and $\Gamma_{\mathrm{m}}$ are conditional, that is to say, depending on the conditions of the experiment (surfactant concentration range and environmental conditions). Literature examples of reasonable fits of the L-eq. for surfactant 
2093

2094

2095

2096

2097

2098

2099

2100

2101

2102

2103

2104

2105

2106

2107

2108

2109

2110

2111

2112

2113

2114

2115

2116

2117

adsorption are mostly based a limited surfactant concentration range around the CMC where the plateau adsorption is reached.

The Frumkin-Fowler-Guggenheim (FFG) equation is an extension of the L-eq. in which lateral interaction between the adsorbed molecules is taken into account; it can be written as

$$
\frac{\theta}{1-\theta}=K c \exp (-b \theta) \quad \text { with } \theta=\frac{\Gamma}{\Gamma_{m}}
$$

where $b$ is a dimensionless lateral interaction parameter: $b=\Delta G_{l a t}^{0} / R T$ with $\Delta G_{l a t}^{0}$ the standard Gibbs energy of lateral interaction. This equation is more realistic for surfactant adsorption than the L-eq. because next to the interaction with the surface $(K)$, the lateral hydrophobic attraction is included explicitly; the parameter $b$ can be expressed as linearly dependent on the alkyl chain length. In many studies of surfactant adsorption FFG-type models have been used to qualitatively understand the role of surface-surfactant attraction as compared to the lateral hydrophobic attraction.

When the binding sites are heterogeneous and randomly or regularly mixed the adsorption isotherm can be obtained by solving the integral adsorption equation that contains both the local isotherm for binding on equal energy sites and the heterogeneity distribution. A Freundlich-type isotherm equation is obtained when the local isotherm is L-type and the heterogeneity distribution is given by a quasi-Gaussian distribution function [57]:

$$
\frac{\theta}{1-\theta}=(\tilde{K} c)^{p} \quad \text { with } \theta=\frac{\Gamma}{\Gamma_{m}}
$$

where $\tilde{K}$ is the median value of the affinity and $p(0<p \leq 1)$ is a measure of the width of the distribution, for $p=1$ the surface is homogeneous. The quasi-Gaussian distribution function is called ‘Sips-distribution’ after Sips [229, 230] who derived Eq. 4. Eq. 4 is called the Langmuir-Freundlich equation, for $p=1$ it reduces to the L-eq.. Because of the presence of an exponent in the equation the use of a dimensionless solute concentration is a must, it makes the interpretation of $K$ possible. For sorbed amounts much lower than $\Gamma_{\mathrm{m}}$ Eq. 4 
2118

2119

2120

2121

2122

2123

2125

2126

2127

2128

2129

2130

2131

2132

2133

2134

2135

2136

2137

2138

2139

2140

2141

reduces to the common Freundlich equation, $\Gamma=\Gamma_{m}(\tilde{K} c)^{p}=K^{*} c^{p}$, where $K^{*}$ is a composite parameter that can be indicated as the effective affinity constant; it has the same dimension as $\Gamma$, (provided $c$ is normalized) but information on $\Gamma_{m}$ cannot be obtained. Freundlich type equations are more 'flexible' than the L-eq.; therefore, they have been mainly applied when the L-eq. did not fit the results well. The interpretation of the obtained parameters is however not so easy. Below we return to this aspect.

So far electrostatic interactions are not considered explicitly; in mean-field models these interactions can be formally introduced in the mentioned models by replacing the (normalized) equilibrium concentration of solute by the equilibrium solute concentration adjacent to the adsorption site:

$$
c_{s}=c_{b} \exp \left(\frac{-z F \psi_{s}}{R T}\right)
$$

where $c_{\mathrm{s}}$ is the solute-ion concentration at the location of the binding site, $c_{b}$ the normalized equilibrium solute concentration in the dilute bulk solution, the Boltzmann factor accounts for the smeared-out electrostatic interaction at the location of the binding sites (mostly the surface) that the solute ion experiences, $z$ is the valence of the solute ion and $\psi_{s}$ is the electrostatic potential at the location of the site. The value of $c_{b}$ can be multiplied by the activity coefficient calculated with, for instance the Debye-Hückel or Davis model [231], when the ionic strength is such that activity corrections are necessary.

A problem with Eq. 5 is that mostly no adequate information is present on $\psi_{\text {s. }}$ For low and moderate ionic strength values the zeta-potential, obtained with one of the electrokinetic methods [204], can be used as approximation for $\psi_{s}$. Alternatively, a model can be used that the relates the particle charge to $\psi_{s}$, For flat surfaces and not too high surface potentials the Debye-Hückel model can be used [79], it relates $\psi_{s}$ to the total (net) surface charge density, $\sigma_{t}(\mathrm{C} / \mathrm{m} 2)$ : 


$$
\psi_{s}=\frac{\sigma_{t}}{\varepsilon_{0} \varepsilon_{r} \kappa}
$$

where $\kappa$ is the inverse Debye length, $\varepsilon_{0}$ the permittivity in vacuum and $\varepsilon_{r}$ the relative permittivity of water. The value of $\kappa$ is determined by the ionic strength of the solution, at room temperature and a z-z electrolyte of concentration $c_{\mathrm{z}}$

$$
\kappa=\left(10 c_{z} z_{z}^{2}\right)^{0.5} \text { with } \kappa \text { in } \mathrm{nm}^{-1} \text { and } \mathrm{c}_{\mathrm{z}} \text { in } \mathrm{mol} / \mathrm{L}
$$

Substitution of Eqs. 5-7 in Eq. 3 provides an equation that explicitly takes into account the specific interaction with the surface, the electrostatic interactions both with the surface and in the adsorbed layer and the lateral interactions:

$$
\frac{\theta}{1-\theta}=K c \exp \left(-b \theta-\frac{z F \sigma_{p}+z^{2} F^{2} \Gamma_{m} \theta}{R T \varepsilon \kappa}\right)
$$

where use has been made of the fact that the total (net) particle charge density $\sigma_{t}=\sigma_{p}+z F \Gamma_{m} \theta$ with $\sigma_{p}$ the primary surface charge density and $z F \Gamma_{m} \theta$ the charge density contribution due to the ionic solute adsorption. This type of equation can be used to explain the presence of a common intersection point (CIP) of ionic surfactant isotherms measured at different ionic strength values. At the CIP the electrostatic interaction vanishes, as there is no effect of the salt concentration on the adsorption. This must mean that $z F \sigma_{p}+z^{2} F^{2} \Gamma_{m} \theta=0$, i.e., the adsorbed surfactant charge just compensates the primary surface charge. For concentrations below the CIP the primary particle charge dominates and an increase in ionic strength (increase in $\kappa$ ) decreases the electrostatic surface-surfactant attraction, therefore the adsorption decreases with increasing ionic strength. For concentrations beyond the CIP the surfactant adsorption dominates the electrostatic interaction and with an increase in ionic strength the repulsion between the charged surfactant head groups decreases, therefore the adsorption increases with increasing ionic strength. From the above equations Eq. 8 is best equipped to understand qualitative or 
2165 (semi)-quantitative ionic surfactant adsorption behavior. Eq. 8 is a FFG-type equation this 2166 can be shown by regrouping the various terms:

$$
\frac{\theta}{1-\theta}=K K_{e l} c \exp \left(-b \theta-b_{e l} \theta\right)
$$

where $K_{e l}=\exp \left(-z F \sigma_{p} / R T \varepsilon \kappa\right)$ and $b_{e l}=\exp \left(-z^{2} F^{2} \Gamma_{m} / R T \varepsilon \kappa\right)$. Eq. 9 shows that

when the electrostatic interactions are not explicitly considered with ionic surfactant adsorption and the simple FFG- Eq. (3) is applied, that both the affinity and the lateral interaction parameter contain an electrostatic contribution. Further extensions of Eqs. 3, 6, 8 and 9 are discussed in [232, 233].

Ionic surfactant adsorption is often at least partly due to ion exchange and/or accompanied by adaptation of the primary surface charge. None of the above equations considers the multicomponent nature or the competition. This implies that when the equations are applied in practice they are conditional, or stated differently, the fitted parameters are no longer adequate when the concentration of the competing ion (e.g., $\mathrm{H}^{+}$or $\left.\mathrm{OH}^{-}\right)$is changed. This can be illustrated by using the multicomponent Langmuir equation:

$$
\theta_{1}=\frac{K_{1} c_{1}}{1+K_{1} c_{1}+K_{2} c_{2}}=\frac{K_{1}^{c} c_{1}}{1+K_{1}^{c} c_{1}} \quad \text { with } K_{1}^{c}=\frac{K_{1}}{1+K_{2} c_{2}}
$$

where $K_{1}^{c}$ is the conditional affinity, which is only constant at constant $c_{2}$. Eq. 10 is also

2181 Langmuir-type but it provides information on how $K_{1}^{c}$ changes with changing conditions. A condition for the application of Eq. 10 is that $\Gamma_{m}$ should be the same for both components; otherwise also the stoichiometry of the exchange has to be taken into account. hydrophobic attraction play a role, and the second step is governed by hydrophobic 2187 attraction to already adsorbed molecules and lateral hydrophobic and electrostatic 
2188 interaction in the second layer. With the above single layer models this distinction is not

2189 made, which implies that the second layer adsorption can only be due to lateral interaction.

2190 As a result the $b$ parameter has a composite character, it accounts for both the layer-layer

2191 and the lateral hydrophobic attraction. A simple model that tries to avoid this problem is the

2192 FFG two-layer model of Mehrian et al. [180] in which not only the affinities for the first and

2193 second layer are different but also the lateral interactions, however the electrostatic

2194 interactions are not made explicit. The results of this model are discussed in the section on

2195 cationic surfactant adsorption to kaolinite. Scamehorn et al. [234] also used a two-layer

2196 FFG equation without treating the electrostatic interactions explicitly. With their parameter

2197 choice the affinity for the first and second layer differed, but the lateral interactions were

2198 assumed to be the same. With the chosen parameter values the model predicted a strong 2D

2199 phase transition at low surfactant concentrations and heterogeneity was invoked to explain

2200 the experimental isotherms. As explained above for the FFG-eq., also a two-layer FFG

2201 model, that does not treat electrostatics explicitly, is only suited for the description of

2202 experiments done at constant $\mathrm{pH}$ and constant salt concentration.

2203

2204

\subsection{Aggregation models for monocomponent solute adsorption}

2205 The second group of adsorption models starts with the Hill equation that describes the 2206 formation of homogeneous aggregates containing $n$ solute molecules on $N_{s}$ homogeneous 2207 binding sites:

2208

$$
\frac{\theta_{n}}{1-\theta_{n}}=(K c)^{n} \quad \text { with } \theta_{n}=\frac{\Gamma_{n}}{\Gamma_{t}}=\frac{\Gamma_{n}}{n N_{s}}
$$

2209 where $\theta_{n}$ is the fraction of sites covered with $n$ molecules, $K$ is the affinity of the binding of 22101 molecule, $K^{n}$ that of the $n$ molecules, $c$ the normalized solute concentration, the actual

2211 adsorption equals $\Gamma_{n}$, and the total (maximum) adsorption $\Gamma_{t}$ is equal to $n N_{s}$. The process of 2212 adsorption is cooperative because one adsorption step brings $n$ molecules to a site, $n$ is 
2213 therefore also called the cooperativity parameter. The Hill-eq. reduces to the L-eq. for $n=1$;

2214 isotherms for $n>1$ are somewhat S-shaped and the isotherm is steeper than the L-isotherm

2215 (positive cooperativity); for high $n$ the steepness of the adsorption isotherm is large and 2216 close to a two-dimensional (2D) phase transition. It is also possible to derive the Hill-eq. for 2217 the situation that one molecule occupies $n^{*}$ sites ( $n^{*}$-dentate adsorption), so that on one site 2218 only $1 / n^{*}$ molecules are adsorbed. In this case $n=1 / n^{*}$ or $n<1$, i.e., for $n=1 / 2$ the 2219 adsorption is bidentate. For $n<1$ there is negative cooperativity and the isotherm is less 2220 steep than the Langmuir isotherm because it more difficult to find $n^{*}$ sites than 1 site. 2221 Instead of calling $n$ the cooperativity parameter, $n$ can thus also be called the stoichiometry 2222 parameter: $n>1$ more than 1 molecule per site, $n<1$ more than 1 site required to adsorb 1 2223 molecule.

2224 It should be noted that the mathematical forms of the Hill-eq. and the 2225 Langmuir-Freundlich-eq. are identical; in terms of the Hill-eq. heterogeneity is a form of 2226 negative cooperativity, where adsorption becomes more difficult when the coverage 2227 increases. When the Hill-eq. or the LF-equation is applied in a practical situation the 2228 cooperativity parameter might therefore be affected by site heterogeneity, and the 2229 heterogeneity parameter by the cooperativity.

2230 Zhu and $\mathrm{Gu}[150,151]$ have proposed an extension of the Hill-eq., they make a 2231 distinction between a first and second step in the adsorption process. In the first step solute 2232 molecules are bound to the sites and in the second steps these bound molecules act as nuclei 2233 for an aggregation step or positive cooperative adsorption. The adsorbed amount in the first 2234 state equals

2235

$$
\Gamma_{1}=N_{s} \theta_{1}=N_{s} \frac{\kappa K c}{1+\kappa K c+\kappa(K c)^{n}}
$$

2236 that in the second state 


$$
\Gamma_{n}=N_{s} n \theta_{n}=N_{s} \frac{n \kappa(K c)^{n}}{1+\kappa K c+\kappa(K c)^{n}}
$$

2238

and the total adsorption is obtained by summation of the two contributions

$$
\Gamma_{t}=N_{s} \frac{k K c+n k(K c)^{n}}{1+k K c+k(K c)^{n}}
$$

In these equations $k$ is the ratio between the affinities of the first and second state, $K$ the affinity of the second state, therefore, $k K$ is the binding affinity for the first state. For $k=1$ the affinities for the first and the second state are equal, but the nuclei formation is initially stronger than the aggregation because there are more free sites than nuclei. For $k>>1$ the isotherm is typical stepwise, the first step is pronounced at low concentration and the second step occurs at somewhat higher concentrations. The parameter $n$ is in this case equal to the ratio of the first adsorption plateau over the second. For $k<<1$ the equation becomes similar to the Hill equation and the isotherm depicts one step. Eq. 12 is often called the Gu-equation, it is a very 'flexible’ equation and regularly used to describe quantitatively the adsorption of both nonionic and ionic surfactants.

In the case of ionic surfactants the description is conditional, because for each change in solution conditions ( $\mathrm{pH}$ and ionic strength) new parameters are required. Moreover, the affinity for the first step is for ionic surfactants determined by both the specific and electrostatic interaction and lateral interactions, the parameter $k$ accounts for the first two interactions, $K$ for the lateral attraction. Ion competition for the surface sites is not considered. The affinity in the second step comprises the hydrophobic affinity for the nuclei and the lateral interactions; the latter contain for ionic surfactants not only the hydrophobic attraction but also the electrostatic repulsion. Part of the lateral interaction for the second step is however also incorporated in the value of $n$. It is therefore difficult to give a more detailed interpretation of the parameter values. The main advantage of the Eq. 12 is that a distinction can be made between the overall affinities for the first and the second step. 
2261 When it is assumed that the adsorbed states are governed by the same electrostatic

2262 adsorption potential it is possible to incorporate the electrostatic interactions in the Gu-eq.

2263 in the same way as illustrated above for the FFG-eq., but this has not been done.

2264 Two sophisticated models for surfactant adsorption that take aggregation into account are 2265 that of Rudzinski et al. [235, 236] and Li and Ruckenstein [237]. The model by Li and 2266 Ruckenstein [237] is most advanced, it has much in common with the description of 2267 micellization of ionic surfactants but it also reflects the scaled particle theory used by 2268 Rudzinski et al. [235, 236]. Solvent molecules, surfactant monomers and two types of 2269 surfactant aggregates of various sizes cover the surface. The competition between the 2270 enthalpic and entropic contributions to the Gibbs energy in the adsorbed phase is 2271 responsible for the composition of the adsorbed phase; the standard Gibbs energy change in 2272 going from the solution to the surface aggregates is calculated by considering five 2273 contributions: hydrophobic, conformational, electrostatic, steric and interfacial. The 2274 electrostatic contributions are treated within the framework of the Poisson-Boltzmann 2275 equation for flat plates. The model can well predict the four-region isotherms, but no 2276 predictions have been made of ionic strength effects.

\subsection{SCFA model for surfactant adsorption}

The SCFA or Self-Consistent-Field theory for Adsorption model is a sophisticated 2280 model in which the structure and properties of the components present in the system are 2281 explicitly taken into account by considering them as composed of different segments that 2282 are linked together. The type and number of segments of a molecule, their charge and the way they are linked determine the structure and the properties of a molecule. Most molecules in the system are composed of just one or a few segments, but the molecular structure of the surfactant molecules is mimicked as closely as possible. The flat surface is also composed of segments that characterize the surface, for charged surfaces a part of the 
2287

2288

2289

2290

2291

2292

2293

2294

2295

2296

2297

2298

2299

2300

2301

2302

2303

2304

2305

2306

2307

2308

2309

2310

2311

2312

segments can associate or dissociate a proton. The model is an extension of the theory of polymer solutions by Flory [107] to systems that have inhomogeneities in one direction (1D SCFA), i.e. perpendicular to the surface or the center of the lattice, or in two directions (2D SCFA), i.e. perpendicular to the surface and in concentric rings parallel to the surface. In the 1D SCFA option a mean field approximation is used in every lattice layer around to the center of the lattice or parallel to the surface. In the 2D case the mean field approximation is used in every ring. The interactions between the segments are calculated using Flory-Huggins interaction parameters, similarly as in the Flory theory. Starting from system characteristics such as the structure of the surface, the number of different types of molecules, the amount of each of them, their structure and properties, and the interactions between the various segments, the equilibrium distribution of molecules in the adsorbed layer is calculated. The conformational statistics of the chains in the layer are evaluated using Boltzmann statistics, i.e., each step of the walk is weighted with a Boltzmann factor that accounts for the interactions that the segment experiences in the adsorbed layer. The electrostatic interactions in the adsorbed layer are calculated using a multi-plate condenser model. In the absence of surfactants the electrostatic potential profiles correspond with the potential calculated with the Stern-Gouy-Chapman model. The detailed equilibrium distribution of surfactant segments within the adsorbed layer, i.e. within a potential field exerted by the presence of the surfactant molecules themselves and the surface, is calculated by an iterative minimization of the Gibbs energy of the system. At equilibrium the segment density distribution in the adsorbed layer and the potential field exerted by the segments and the surface are fully in accordance with each other (field is self-consistent). Once this is achieved the structure of the adsorbed layer and the thermodynamic properties of the system can be obtained. Similarly as in the model of Li and Ruckenstein [237], the SCFA theory has a closely related variant for self-assembly in solution [238, 239] so that the surfactant micellization can also be predicted. When micellization of a given surfactant is predicted 
2313 with a certain set of parameters, the same set of parameters should be used to predict the

2314 adsorption. The only new parameters for the adsorption calculation are in that case those

2315 that describe the interactions of the segments with the solid surface. As mentioned in the

2316 text on several places, the SCFA model has been very helpful for a better understanding of

2317 the surfactant adsorption behavior. Reference [25] is a fairly recent review in which

2318 experimental surfactant adsorption results obtained for metal oxides and silicas are

2319 compared with SCFA calculations. More recent results for surfactant adsorption in confined

2320 space can be found in the section on cationic surfactant adsorption on montmorillonite. For

2321 the SCF calculations a computer code, the SF-box, is available; for further information

2322 and/or application of the SF-Box see [240].

2323

2324

\subsection{NICA-Donnan model applied to surfactant sorption on humic and fulvic acids}

2325

Surfactant sorption to humic and fulvic acids can be described with the NICA-Donnan

2326 model as an ion exchange process. In this model the humic substance is considered as a collection of particles that contain the binding sites and that are permeable for solvent and solute. The particles form the Donnan phase in which the electrostatic interactions are governed by a smeared-out electrostatic potential that is the result of the fixed charges and the mobile charges in the Donnan phase. The binding to the heterogeneous sites is described

2331 by the NICA model, which is based on the multicomponent Hill equation as local isotherm 2332 in combination with the Sips distribution and can be written as $[57,58]$

$$
Q_{i}=Q_{H}^{\max }\left(\frac{\tilde{n}_{i}}{\tilde{n}_{H}}\right) \frac{\left(\tilde{K}_{i} c_{D, i}\right)^{\tilde{n}_{i}}}{\sum_{i}\left(\tilde{K}_{i} c_{D, i}\right)^{\tilde{n}_{i}}} \cdot \frac{\left(\sum_{i}\left(\tilde{K}_{i} c_{D, i}\right)^{\tilde{n}_{i}}\right)^{p}}{1+\left(\sum_{i}\left(\tilde{K}_{i} c_{D, i}\right)^{\tilde{n}_{i}}\right)^{p}}
$$

2334 where $Q_{H}{ }^{\max }$ is the maximum sorption of protons, $\tilde{n}_{i}$ the Hill-parameter that accounts for the

2335 average stoichiometry of component $i$ with the proton sites, $\tilde{K}_{i}$ is the median affinity of 
2336

2337

2338

2339

2340

2341

2342

2343

2344

2345

2346

2347

2348

2349

2350

2351

2352

2353

$Q_{j}=\Omega\left(\tilde{K}_{j} c_{D, j}\right)^{\tilde{n}_{j}}$ with $\Omega=Q_{H}^{\max }\left(\frac{\tilde{n}_{i}}{\tilde{n}_{H}}\right) \frac{1}{\sum_{i}\left(\tilde{K}_{i} c_{D, i}\right)^{\tilde{n}_{i}}} \cdot \frac{\left(\sum_{i}\left(\tilde{K}_{i} c_{D, i}\right)^{\tilde{n}_{i}}\right)^{p}}{1+\left(\sum_{i}\left(\tilde{K}_{i} c_{D, i}\right)^{\tilde{n}_{i}}\right)^{p}}$

component $i$ for the sites and $p$ the heterogeneity parameter. It is assumed that the first characterization of the humic substance is done through proton sorption, therefore this ion is used as reference for the total number of sites $\left(Q_{H}^{\max }\right)$ and the factor $\tilde{n}_{i} / \tilde{n}_{H}$ accounts for the fact that the maximum sorption of $i$ can be different from that of the proton. The concentration $C_{D, i}$ is the concentration of $i$ in the Donnan phase that can be obtained from the bulk solution concentration using Eq. 5 with $\psi_{s}=\psi_{D}$. In the Donnan model the charge of the humic particles is neutralized by counter- and co-ions within the Donnan volume, the latter is calculated with a simple empirical relation. Based on this electroneutrality the Donnan potential can be calculated and $c_{D, i}$ can be obtained for all ions present [38]. The NICA equation is a master equation that reduces to the multicomponent Langmuir equation for all $\tilde{n}_{i}=1$, and $p=1$, to the multicomponent Langmuir -Freundlich eq. for all $\tilde{n}_{i}=1$ and to the multicomponent Hill eq. for $p=1$.

The last quotient in Eq.13 is the total coverage of the sites with all types of ions present in the system, the second quotient is the fraction of the total coverage that is occupied by component i. Therefore, in the case of sorption of a trace component, $j$, $\left(\left(\tilde{K}_{j} c_{D, j}\right)^{\tilde{n}} j \ll \sum_{i}\left(\tilde{K}_{i} c_{D, i}\right)^{\tilde{n}}{ }^{i}\right)$ the total coverage is hardly affected by the binding of species $j$ and the NICA eq. reduces to

2354 where $\Omega$ is considered to be constant. When the total adsorption is constant, then also the

2355 Donnan potential will be constant and the electrostatic interactions do not significantly 2356 change with sorption of $j$, thus also $c_{D, j}$ can be replaced by $c_{j}$ (Boltzmann factor becomes 2357 included in $\Omega$ ). Therefore, at low solute concentrations in a multicomponent system the 2358 sorption equation of a trace ionic component to the active sites of the humic substance, at 
2359 otherwise constant solution conditions ( $\mathrm{pH}$, ionic composition and ionic strength) is ruled 2360 by a simple equation that resembles the monocomponent Hill-eq. for low loadings. Because 2361 the sorption of $j$ is low, the site heterogeneity is not detected but the cooperativity / 2362 stoichiometry ( $\tilde{n}_{j}$ ) is relevant. When the humic substance is well studied, $\Omega$ is known and 2363 the changes of $\Omega$ with changing solution conditions (e.g., change of $\mathrm{pH}$ ) can be calculated, 2364 therefore the behavior of the trace component with changing solution conditions can be 2365 predicted, once $\tilde{K}_{j}$ and $\tilde{n}_{j}$ are known. When no information is present on the humic 2366 acid, the generic humic acid proposed by Milne et al. [55, 56] can be used to represent the 2367 unknown humic acid and by using the parameters collected by Milne the total sorption can 2368 be calculated under the given conditions. Thus also in this case Eq. 14 can be used in 2369 combination with Eq. 5 to investigate trace component sorption and how it is affected by, 2370 e.g., $\mathrm{pH}$.

2371 In the case of metal ion binding a double NICA-Donnan equation has been used, where 2372 the first equation accounts for the low proton affinity sites ('carboxylic') and the second for 2373 the high affinity proton sites ('phenolic'). The above way of analyzing trace component 2374 binding is still relevant as long as the total sorption on both the phenolic and carboxylic type 2375 of groups is constant. In most cases the trace sorption will occur with either the carboxylic 2376 or the phenolic groups and in this case the type of groups that do not participate can be 2377 neglected.

2378 For the surfactant binding only a limiting cases of the single NICA-Donnan equation 2379 have been used. As discussed in the section 'Modeling surfactant - humic substance 2380 interaction' Chen et al. [114] used Eq. 14 to unravel the surfactant binding and the Hill 2381 equations used by Yee et al. [98-101] are identical to Eq. 14. Ishiguro and Koopal [115] 2382 made a different simplification for their analysis of surfactant adsorption to various humic 
2383 acids; they assumed that the surfactant sorption was dominant, i.e., $2384\left(\tilde{K}_{j} c_{D, j}\right)^{\tilde{n}} j \approx \sum_{i}\left(\tilde{K}_{i} c_{D, i}\right)^{\tilde{n}_{i}}$. In that case the NICA eq. reduces to

$$
Q_{j}=Q_{j}^{m a x} \frac{\left(\tilde{K}_{j} c_{D, j}\right)^{m}}{1+\left(\tilde{K}_{j} c_{D, j}\right)^{m}} \quad \text { with } m=\tilde{n}_{j} p
$$

2386

Eq. 15 is mathematically equivalent to the Langmuir-Freundlich equation, but here the exponent is a composite parameter that reflects both the cooperativity and the heterogeneity. Because the surfactant sorption covered the entire sorption range up to $Q_{j}^{\max }$ the exponent in Eq. 15 is also affected by the heterogeneity. For the calculation of the Donnan potential the total (net) charge of the humic acids was used and the proton charge adjustment due to the surfactant adsorption was accounted for. When the charge adjustments are not known and a computer code for NICA-Donnan calculations is available, a good method is to use the full (single) NICA-Donna equation together with humic substance specific parameters or generic humic substance parameters $[55,56]$, because then the charge adjustments are automatically taken into account. Computer codes that include the NICA-Donnan model are ECOSAT [241] and Visual MINTEQ ver. 3.0 / 3.1 [242]. Furthermore, the computer code 'Fit' [243] is designed to fit experimental results to a range of adsorption isotherm equations.

\section{Surfactant adsorption on soils}

\subsection{General considerations and trends}

Soils are composed of organic and inorganic components each with different adsorption characteristics as discussed in the previous sections. Therefore, the adsorption of a surfactant on a soil sample will depend on both the types of soil constituents and their relative proportions. This has also been stated in literature [244]. The overall surfactant 
2407

2408

2409

2410

2411

2412

2413

2414 $2415[50]$.

2416

2417

adsorption to a given soil can be thus be approximated by a weighted summation of the adsorptions to the individual constituents, or oppositely, the soil composition has to be known to be able to understand the surfactant adsorption behavior. Yet, the overall adsorption is not necessarily equal to the sum of the adsorptions to the different components because the soil components may mutually interact and this interaction will affect the adsorption. The mutual interaction is especially relevant and strong in the case of net positively charged metal oxides or layered silicates and the negatively charged humic and fulvic acids [245-249]. A similar situation is encountered with trace element binding to soils

Some general trends have also been observed for surfactant adsorption to soils. Hydrophobic interaction is important with surfactant adsorption. Due to increasing hydrophobic attraction surfactant adsorption increases, in general, with increasing organic matter content of the soils [250-256]. Treatment of a soil with $\mathrm{H}_{2} \mathrm{O}_{2}$ to remove the organic matter will therefore, in general, decrease the surfactant adsorption [228, 229], but the contrary has also been observed [231]. Nonionic surfactants bind to soil surfaces with hydrogen bonding on the $-\mathrm{OH}$ sites of soils besides hydrophobic interaction [257], but hydrogen bonding is also possible to the hydrated cations on siloxane basal planes [198]. Longer alkyl chains lead to stronger lateral hydrophobic attraction, this explains that there is also a positive correlation between aliphatic chain length and surfactant adsorption on soils. An example of this behavior has been presented by Westall et al. [255] who showed that linear alkylbenzene sulfonates with longer carbon chains adsorbed stronger on a sediment (Fig. 29). Adsorption studies comparing linear and branched aliphatic chain structures have also been performed for soils and alumina [258-261]. For surfactants with the same number of $\mathrm{CH}_{2}$ segments, branching leads to lower adsorbed amounts at the same surfactant concentration. This is largely due to a decrease of the hydrophobic attraction; the decrease in adsorption corresponds with the increase in CMC with branching [261]. 
Soils possess both permanent and pH-dependent charges [262, 263]. The contents of those charges differ among soils and affect the adsorption of charged surfactants strongly. Soils and sediments generally have negative charges, therefore, the adsorption of anionic surfactants on soils and sediments is much lower than that of nonionic and cationic surfactants [251, 255, 256, 264, 265]. Bera et al. [266] showed that surfactant adsorption on a sand was in the order of cationic $>$ nonionic $>$ anionic (Fig. 30). For surfactants with a similar alkyl chain the adsorption of the cationic surfactant is largest because of the electrostatic attraction between surface and surfactant and that of the anionic surfactant is smallest because of the electrostatic repulsion. An example where the relatively low anionic surfactant adsorption to sand is applied is surfactant-enhanced soil remediation. With surfactant-enhanced soil remediation, surfactant adsorption to the soil particles increases the surfactant use and this decreases the efficiency. Therefore, anionic surfactants have usually been chosen in these technologies [267-269]. However, nonionic surfactants are also often used for the remediation because of their lower CMC compared to ionic surfactants, higher degree of surface tension reduction, and relatively constant properties in the presence of salt $[268,269]$.

A positive correlation is observed between cationic surfactant adsorption and the soil CEC because the CEC characterizes the soil negative charge density and the latter generates the electrostatic attraction with cationic surfactants [270]. For the soils which possess variable charges the $\mathrm{pH}$ also affects the adsorption as shown in Fig. 31 [266]. Anionic surfactant adsorption decreases as pH increases [254, 255, 258-260, 266, 271] because the soil negative charge increases and/or the positive charge decreases. On the other hand, the opposite occurs for cationic surfactant adsorption as shown in Fig. 31 [266]. When after sodium citrate - bicarbonate - dithionite treatment the fraction amorphous oxides in soils is decreased, the adsorption of anionic surfactant also decreases because the density of positively charged sites of the soils decreases [260]. Nonionic surfactant adsorption 
2459 increases as $\mathrm{pH}$ decreases at low $\mathrm{pH}$ and remains almost constant at high pH (Fig. 31) [266].

2460 The increase at low $\mathrm{pH}$ resulted from the increase of the surface hydroxyl sites (due to 2461 protonation of the surface oxygens) to which the EO segments of the nonionics can bind by 2462 hydrogen bonding.

2463 The electrolyte concentration also affects ionic surfactant adsorption. It is observed that 2464 anionic surfactant adsorption on negatively charged soils increases, in general, with the 2465 increase of electrolyte concentration [255, 258, 266]. This must be due to a larger screening 2466 of both the electrostatic repulsion between surface and surfactant and the mutual 2467 electrostatic repulsion between the adsorbed surfactant ions. Increase of cationic surfactant 2468 adsorption on negatively charged soils with the increase of electrolyte concentration is also 2469 observed [272, 273]. This is caused by the screening of the electrostatic repulsion between 2470 the head-out adsorbed head groups of the cationic surfactants. This situation occurs for 2471 bilayer and/or admicelle adsorption where part of the head groups are directed to the soil 2472 solution; this part of the surfactants is adsorbed by hydrophobic attraction to the carbon 2473 chains of the surfactants adsorbed in the first layer. In this case, the cationic surfactant 2474 adsorption is also affected by the type of anion of the electrolyte. The surfactant adsorption 2475 increases in the order of $\mathrm{SO}_{4}{ }^{2-}>\mathrm{Br}^{-}>\mathrm{Cl}^{-}$because the screening effect increases in this order 2476 [272]. The influence of the electrolyte concentration becomes reverse for ionic surfactants 2477 adsorbed in direct contact with the oppositely charged soil surface (head-on adsorption). 2478 The adsorption decreases with the increase of electrolyte concentration due to the screening 2479 of the electrostatic surfactant-surface attraction.

2480 An example of the influence of the electrolyte concentration on the sorption of dodecyl 2481 benzene sulfonate (DBS) on a humic soil is depicted in Fig. 32 [258]. The adsorption 2482 decreases with decreasing electrolyte concentration because the electrostatic repulsion 2483 increases. The influence of the (smeared-out) electrostatic potential at the location of the 2484 adsorption sites can be evaluated by using the Langmuir-Donnan model. In this model it is 
assumed that the soil particles are covered with humic substances and that the surfactant binds to the sites in the humic layer according to a Langmuir equation that is extended by including the electrostatic attraction by a Boltzmann factor, $\exp \left(-z F \psi_{D} / R T\right)$, where $\psi_{D}$ is the Donnan potential in the humic layer (see section 7):

$$
Q_{j}=Q_{j}^{\max } \frac{K^{\prime} c_{D, j}}{1+K^{\prime} c_{D, j}}
$$

$$
c_{D, j}=c_{b, j} \exp \left(\frac{-z_{j} F \psi_{D}}{R T}\right)
$$

where, $Q_{j}$ is the surfactant adsorption, $Q_{j}^{\max }$ the maximum surfactant adsorption, $K$ ' the specific or chemical affinity constant (surface-surfactant), , $z_{j}$ the valence of the surfactant ion, $c_{D, j}$ the surfactant concentration in the Donnan phase and $c_{b, j}$ the surfactant concentration in the bulk solution. When for all salt concentrations the value of $Q_{j}^{\text {max }}$ is put equal to the surfactant sorption at the CMC (estimated to be $20 \mu \mathrm{mol} / \mathrm{g}$ ), the affinity constant, $K^{\prime}$, and the Donnan potentials, $\psi_{D}$, can be obtained by fitting Eq. $16+17$ to the experimental data in the region where the slope of the double logarithmic plots is about equal to unity (region where Eq. 16 applies). The fitted adsorption isotherms and obtained Donnan potentials are depicted in Fig. 32. The negative value of Donnan potential increases at constant salt concentration due to the fact that DBS sorption increases the negative charge of the particles. Increasing the salt concentration at a given DBS sorption has the opposite effect: the salt ions screen the particle charge; therefore, the repulsive Donnan potential decreases with increasing the salt concentration. At the lowest surfactant concentrations the isotherms rise very steeply; this likely indicates strong sorption to some highly active sites of the soil sample.

\subsection{Fate of surfactants in soils; degradation, hysteresis and precipitation}


When the fate of surfactants in soil and water environments is considered, surfactant 2509 degradation is important, as well as adsorption. However, different classes of surfactants have different degradation behavior in the environment [264]. Sodium dodecylsulfate (SDS) easily degrades, especially in humic soil. Therefore, the influence of degradation on adsorption experiments is significant. Results of some batch experiments of SDS in the presence of a highly humic non-allophanic andosol are depicted in Fig. 33 [274]; the sum of adsorption and degradation corresponds to the SDS decay in the soil solution. The “adsorption + degradation” amount at intermediate SDS concentrations increases with time due to increasing of degradation. The initial steep increase is somewhat puzzling; the irregular behavior suggests that either or both degradation and cooperative adsorption are important in this region [274].

The environment also affects the degradation; sodium dodecylbenzenesulfonate with a linear carbon chain (L-DBS) degrades in river water with a half-life of less than 3 days, but its degradation in aerobic soil with a half-life of 7-33 days is much longer [264]. In general, adsorption restricts L-DBS degradation, especially in soils with allophane and amorphous metal oxides [260], which adsorb the anionic surfactant by electrostatic attraction. and the desorption isotherm [168, 169, 275], must be also considered as a distinctive 2526 phenomenon. In general, the adsorbed amount with desorption is larger than that of 2527 adsorption [169, 275]. The hysteresis can, in general, be due to kinetic and/or 2528 multicomponent adsorption effects. An important kinetic reason for the difference between 2529 the adsorption and desorption isotherm is that the kinetics of desorption become very slow 2530 when the adsorption isotherm is rather steep [276]. In this case the adsorption isotherm is 2531 the true isotherm, the desorption isotherm is an artifact. In multicomponent systems the 2532 adsorption conditions are often not the same as the desorption conditions. This is especially 2533 the case in the presence of impurities adsorbed to the solid and when the solid area to 
2534 solution volume ratio is different in the ad- and desorption experiment. In the case of

2535 adsorption by ion exchange ('first layer' adsorption) and hydrophobic attraction (admicelle 2536 formation or 'second layer' adsorption), desorption of the 'second layer' shows no 2537 hysteresis. This has, for instance, been observed for cationic surfactant adsorption on 2538 vermiculitic soil with a negative charge [272].

2539 Ionic surfactant insolubility must be considered when the Krafft temperature is larger 2540 than the temperature under the conditions of the adsorption [201, 277, 278]. The Krafft 2541 temperature or Krafft point is the minimum temperature at which surfactants can form 2542 micelles, below the Krafft temperature surfactant added to a solution with a monomer 2543 concentration close to the CMC remains in the crystalline form. Therefore, micelles are not 2544 formed when the temperature is lower than the Krafft point. The Krafft point becomes 2545 higher when the coion of the surfactant is multivalent.

\subsection{Influence of surfactant adsorption on soil structure and permeability}

Because surfactants modify the surface characteristics of solids, they affect the structure of soils and this affects the water permeability and gas diffusion in soils [279-283]. Proper permeability and gas diffusion are required for crop growth; therefore, a good soil structure must be maintained in crop fields. When a soil disperses and swells during the irrigation or 2552 rainfall, dispersed soil particles clog the macro-pores (water transmission pores) in the soil 2553 and permeability is decreased. A change in soil structure that leads to a decrease in 2554 permeability is a negative factor in relation to agriculture and soil- water environment.

2555 Moreover, sometimes flooded water conveys soils and causes erosion; important soil is lost and the nutrients or chemicals in the eroded soil cause water contamination. The soil dispersion is generally caused by clays and the reduction of hydraulic conductivity is larger 
2559 reduced, the reduction of hydraulic conductivity becomes larger because clays in soils with 2560 a high organic content are not easily dispersed [279].

2561 A review on soil structure can be found in [284]. Soil and clay structural changes with changing inter-particle interaction have been described in [263, 285-288]. In general, repulsive inter-particle interaction allows the particles to slip along each other, which leads to relatively dense structures; with attractive inter-particle interactions the particles stick together which retards further individual movement and the clustering leads to a relatively open structure. It is general knowledge that a settling stable dispersion forms a compact structure and a settling unstable dispersion forms an open structure. The use of surfactants 2568 for soil washing to remove low-solubility organic contaminants, such as oils, also changes 2569 the inter-particle interactions and can result in a significant change in the hydraulic 2570 conductivity of porous media [279, 283]. Electrolyte concentration, $\mathrm{pH}$ and specific ions that all likely affect the particle-particle interaction may also change the soil permeability [263, 288-297]. An example of the effect of surfactant adsorption on soil stability is depicted in Fig. 34 [272], which shows the adsorption isotherm of hexadecyltrimethylammonium $\left(\mathrm{HDTMA}=\mathrm{C}_{16 \mathrm{TA}}{ }^{+}\right.$), on two vermiculitic soils (Na and $\left.\mathrm{Ca}\right)$, and the corresponding effects on the electrophoretic mobility of the soil particles and the optical density of the soil suspension. When the soil is better dispersed, the optical density

2577 becomes larger. For the Na-soil, the shape of the isotherm in region I indicates that the 2578 vermiculite is expanding upon adsorption. Up to about adsorbed amounts equal to 1.0 CEC, 2579 the soil gradually flocculates with increasing surfactant adsorption and the electrophoretic 2580 mobility changes from negative to zero at the IEP, where the surfactant adsorption is about 2581 equal to the CEC. The negative charge of the soil is gradually compensated with the 2582 adsorbed surfactant charge; therefore, the electrostatic repulsion between the soil particles 2583 gradually vanishes and the dispersion is completely flocculated. With a vermiculitic soil and 2584 a cationic surfactant the adsorption occurs initially by ion exchange, this implies surfactant 
2585 head groups in contact with the surface and tails contacting the solution; therefore, also the

2586 hydrophobicity of the soil particles increases and this is likely a second contribution to the 2587 decrease in dispersion stability. When the adsorption becomes larger than the soil CEC 2588 further adsorption will occur by hydrophobic attraction and the formation of an admicellar 2589 layer (second layer) on the surface. With further adsorption the soil particles become 2590 positive and less hydrophobic and the soil disperses again. The positive charge is indicated 2591 by the positive electrophoretic mobilities, For the Ca-vermiculite-soil, the behavior is 2592 different. The shape of the isotherm indicates that there is negligible swelling of the 2593 vermiculite. Moreover, the electrophoretic mobilities are rather low and this explains the 2594 poor initial degree of dispersion. Surfactant adsorption by ion exchange hardly changes the 2595 external particle charge and hence the mobility stays low as does the degree of dispersion. 2596 The Ca-soil begins to disperse after passing the IEP where the particles become positive and 2597 the second adsorption layer starts, but the stability of dispersion stays somewhat lower than 2598 for the Na-soil. The latter may be due to the effect of the released Ca ions on the ionic 2599 strength.

2600 Allred and Brown [279] showed that the soil hydraulic conductivity significantly 2601 decreased with the infiltration of ionic and nonionic surfactant solutions at high 2602 concentration (1 mol/kg); the behavior is depicted in Fig. 35. The curves of N1, N2 and N3 2603 in Fig. 35A represent the nonionic surfactants, the curves of A1 and A2 in Fig. 35B 2604 represent the anionic surfactants, the curves of C1 and C2 in Fig. 35B represent the cationic 2605 surfactants, and the curve of AM1 in Fig. 35B represents the amphoteric surfactant. The 2606 hydraulic conductivity reduction was larger for ionic surfactants than nonionic surfactants. 2607 When an anionic surfactant is adsorbed on a negatively charged soil, the negative charge of 2608 the soil surface becomes larger and the soil particles disperse easier, and the particles slip 2609 easier along each other; therefore, the hydraulic conductivity decreases. When a cationic 2610 surfactant is adsorbed on the negatively charged soil, the situation is somewhat similar to 
2611 that described in the previous section for the vermiculite soils. The stability of dispersion

2612 goes through a minimum, but when the positive charge due to surfactant adsorption is

2613 sufficient the soil particles become well dispersive and the hydraulic conductivity tends to

2614 decrease. When the electrolyte concentration is large, the influence of ionic surfactant

2615 adsorption on the decrease of hydraulic conductivity is restricted [281], because the soil

2616 dispersion ability is inhibited by the strong screening of the particle-particle repulsion.

2617 As a nonionic surfactant does not change the particle charge its effect on the dispersion

2618 stability can only result from the way the surfactant is adsorbed. On most natural surfaces

2619 the hydrophilic head group will be in contact with the surface and similarly as for cationic

2620 surfactants a second layer may become adsorbed at surfactant concentrations around the

2621 CMC. For short EO chains the adsorption in the 'first layer' is moderate and the transition

2622 to the 'second layer' occurs in a narrow concentration range close to the CMC. For long EO

2623 chains the adsorption in the 'first layer' is larger, but due to the large head group it is

2624 difficult for the alkyl chains to associate and only when the alkyl chains are also fairly long

2625 a sufficient 'second layer' will form. Also with kaolinite the edge surface probably

2626 contributes, but on montmorillonite this contribution is relatively small. Therefore, the

2627 behavior with respect to hydraulic conductivity will strongly depend on the type of nonionic

2628 surfactant and the type of soil surface. Mustafa and Letey [298] indicated for a hydrophobic

2629 sandy loam, a hydrophilic sandy loam and a hydrophilic clay loam that nonionic surfactant

2630 adsorption induced soil dispersion by decreasing the aggregate size.

2631 Water infiltration into dry soils with a hydrophobic surface is also affected by surfactant

2632 adsorption. Fig. 36 shows the upward water infiltration into a peat moss column [299]. The

2633 bottom of the air-dried peat moss column was set $20 \mathrm{~cm}$ below the water table. A 70-mM

2634 SDS solution infiltrated faster than pure water because the surface became wettable after the

2635 adsorption of SDS. On the other hand, infiltration of the surfactant solution into dry sand

2636 became slower compared to pure water infiltration, because the sand was already wettable 
2637

2638

2639

2640

2641

2642

2643

2644

2645

2646

2647

2648

2649

2650

2651

2652

2653

2654

2655

2656

2657

2658

2659

2660

2661

2662

2663

2664

2665

2666

2667

2668

2669

2670

without surfactant. In this case, they explained that the surface tension of surfactant solution was smaller than that of pure water and the viscosity of surfactant solution was larger than pure water and both effects decreased the rate of capillary infiltration.

\section{Acknowledgements}

The authors thank Hokkaido University for providing a 'Visiting Professor' grant to LKK for a period of 2 months and the 'Japan Society for the Promotion of Science' for the financial support via 'Grants-in-Aid for Scientific Research (No. 25252042)’.

\section{References}

[1] Koopal LK. Wetting of solid surfaces: Fundamentals and charge effects. Adv Colloid Interface Sci 2012;179:29-42.

[2] Myers D. Surfactant science and technology. 3rd ed: John Wiley \& Sons; 2005.

[3] Tadros TF. Surfactants in agrochemicals: CRC Press; 1994.

[4] Mulligan CN, Yong RN, Gibbs BF. Surfactant-enhanced remediation of contaminated soil: A review. Eng Geol 2001;60:371-80.

[5] Mao XH, Jiang R, Xiao W, Yu JG. Use of surfactants for the remediation of contaminated soils: A review. J Hazard Mater 2015;285:419-35.

[6] Kosswig K. Surfactants. Ullmann's encyclopedia of industrial chemistry: Wiley-VCH; 2002.

[7] McAvoy DC, Eckhoff WS, Rapaport RA. Fate of linear alkylbenzene sulfonate in the environment. Environ Toxicol Chem 1993;12:977-87.

[8] Zoller U. Handbook of detergents: Environmental impact : Surfactant science series 121: CRC Press; 2004.

[9] Jahan K, Balzer S, Mosto P. Toxicity of nonionic surfactants. In: Kungolos AG, Brebbia CA, Zamorano M, (eds). Environmental Toxicology II. Vol. 110: WIT Press; 2008.

[10] Rebello S, Asok AK, Mundayoor S, Jisha MS. Surfactants: Toxicity, remediation and green surfactants. Environ Chem Lett 2014;12:275-87.

[11] McWilliams P. Bioaccumulation potential of surfactants: A review. Aberdeen: European Oilfield Speciality Chemicals Association; 2000. http://www.eosca.com/publications-documents

[12] Scott MJ, Jones MN. The biodegradation of surfactants in the environment. Biochimica et Biophysica Acta (BBA)-Biomembranes 2000;1508:235-51.

[13] Gheorghe S, Lucaciu I, Paun I, Stoica C, Stanescu E. Ecotoxicological behavior of some cationic and amphoteric surfactants (biodegradation, toxicity and risk assessment). In: Chamy R, Rosenkranz F, (eds). Biodegradation - life of science. INTECH; 2013. 
2671

2672

2673

2674

2675

2676

2677

2678

2679

2680

2681

2682

2683

2684

2685

2686

2687

2688

2689

2690

2691

2692

2693

2694

2695

2696

2697

2698

2699

2700

2701

2702

2703

2704

2705

2706

2707

2708

2709

[14] Tan KH. Principles of soil chemistry. 4th ed: CRC Press; 2011.

[15] Schwarzenbach RP, Gschwend PM, Imboden DM. Environmental organic chemistry. 2nd ed: Wiley-Interscience; 2003.

[16] Brackmann B, Hager C. The statistical world of raw materials, fatty alcohols and surfactants. CD Proceedings 6th World Surfactant Congress CESIO, Berlin, Germany2004.

[17] Mulligan CN. Environmental applications for biosurfactants. Environ Pollut 2005;133:183-98.

[18] Bustamante M, Duran N, Diez MC. Biosurfactants are useful tools for the bioremediation of contaminated soil: A review. J Soil Sci Plant Nutr 2012;12:667-87.

[19] Everett DH. Manual of symbols and terminology for physicochemical quantities and units, appendix II: Definitions, terminology and symbols in colloid and surface chemistry. Pure Appl Chem 1972;31:577-638.

[20] Koopal LK. Definitions, terminology and symbols in colloid and surface chemistry , internet version with annotations of dh everett, manual of symbols and terminology for physicochemical quantities and units, appendix II: Definitions, terminology. Web Edition; 1972. http://old.iupac.org/reports/2001/colloid_2001/manual_of_s_and_t/manual_of_s_and_t.html

[21] Matijević E, Pethica B. The properties of ionized monolayers. Part 1.—sodium dodecyl sulphate at the air/water interface. Trans Faraday Soc 1958;54:1382-9.

[22] Matijević E, Pethica B. The properties of ionized monolayers. Part 2.-the thermodynamics of the ionic double layer of sodium dodecyl sulphate. Trans Faraday Soc 1958;54:1390-9.

[23] Matijević E, Pethica B. The properties of ionized monolayers. Part 3.- the thermodynamics of the ionic double layer of sodium octanoate. Trans Faraday Soc 1958;54:1400-7.

[24] Rosen MJ. Surfactants and interfacial phenomena. 3rd ed. New York: Wiley \& Sons; 2004.

[25] Koopal LK. Modeling association and adsorption of surfactants. In: Esumi K, Ueno M, (eds). Structure-performance relationships in surfactants. Surfactant science series Vol. New York: Marcel Dekker; 2003. p. 111-96.

[26] Mukerjee P, Mysels KJ. Critical micelle concentrations of aqueous surfactant systems. Vol. NSRDS-NBS 36: National Institute of Standards and Technology, Washington D.C. USA; 1971.

[27] van Os NM, Haak JR, Rupert LAM. Physico-chemical properties of selected anionic, cationic and nonionic surfactants: Elsevier; 1993.

[28] Nagarajan R, Ruckenstein E. Theory of surfactant self-assembly: A predictive molecular thermodynamic approach. Langmuir 1991;7:2934-69.

[29] Nagarajan R. Molecular packing parameter and surfactant self-assembly: The neglected role of the surfactant tail. Langmuir 2002;18:31-8.

[30] Israelachvili JN, Mitchell DJ, Ninham BW. Theory of self-assembly of hydrocarbon amphiphiles into micelles and bilayers. J Chem Soc Faraday Trans 2 1976;72:1525-68.

[31] Israelachvili J. The science and applications of emulsions-an overview. Colloids Surf A 1994;91:1-8.

[32] Mitchell DJ, Ninham BW. Micelles, vesicles and microemulsions. J Chem Soc Faraday Trans 2 1981;77:601-29. 
2710

2711

2712

2713

2714

2715

2716

2717

2718

2719

2720

2721

2722

2723

2724

2725

2726

2727

2728

2729

2730

2731

2732

2733

2734

2735

2736

2737

2738

2739

2740

2741

2742

2743

2744

2745

2746

2747

2748

[33] Swift RS. Organic matter characterization. In: Sparks D, Page A, Helmke P, Loeppert R, Soltanpour P, Tabatabai M, et al., (eds). Methods of soil analysis part 3-chemical methods. Madison: Soil Science Society of America; 1996. p. 1011-69.

[34] Leenheer JA. Progression from model structures to molecular structures of natural organic matter components. Ann Environ Sci 2007;1:15.

[35] Sutton R, Sposito G. Molecular structure in soil humic substances: The new view. Environ Sci Technol 2005;39:9009-15.

[36] Langford CH, Melton JR. When should humicsubstances be treated as dynamic combinatorial systems? . In: Ghabbour EA, Davies G, (eds). Humic substances: Molecular details and applications in land and water conservation. New York: Taylor \& Francis; 2005. p. 65-78.

[37] Avena MJ, Wilkinson KJ. Disaggregation kinetics of a peat humic acid: Mechanism and pH effects. Environ Sci Technol 2002;36:5100-5.

[38] Koopal LK, Saito T, Pinheiro JP, van Riemsdijk WH. Ion binding to natural organic matter: General considerations and the NICA-donnan model. Colloids Surf A 2005;265:40-54.

[39] Schulten H-R, Schnitzer M. Chemical model structures for soil organic matter and soils. Soil Sci 1997;162:115-30.

[40] Duval JFL, Wilkinson KJ, Van Leeuwen HP, Buffle J. Humic substances are soft and permeable: Evidence from their electrophoretic mobilities. Environ Sci Technol 2005;39:6435-45.

[41] Nederlof MM, De Wit JC, Van Riemsdijk WH, Koopal LK. Determination of proton affinity distributions for humic substances. Environ Sci Technol 1993;27:846-56.

[42] Nederlof MM, van Riemsdijk WH, Koopal LK. Heterogeneity analysis for binding data using an adapted smoothing spline technique. Environ Sci Technol 1994;28:1037-47.

[43] Kinniburgh DG, van Riemsdijk WH, Koopal LK, Borkovec M, Benedetti MF, Avena MJ. Ion binding to natural organic matter: Competition, heterogeneity, stoichiometry and thermodynamic consistency. Colloids Surf A 1999;151:147-66.

[44] Avena MJ, Vermeer AWP, Koopal LK. Volume and structure of humic acids studied by viscometry pH and electrolyte concentration effects. Colloids Surf A 1999;151:213-24.

[45] Tan WF, Koopal LK, Weng LP, van Riemsdijk WH, Norde W. Hurnic acid protein complexation. Geochim Cosmochim Acta 2008;72:2090-9.

[46] Jones MN, Bryan ND. Colloidal properties of humic substances. Adv Colloid Interface Sci 1998;78:1-48.

[47] Tipping E. Cation binding by humic substances. Cambridge (UK): Cambridge University Press; 2002.

[48] Dudal Y, Gérard F. Accounting for natural organic matter in aqueous chemical equilibrium models: A review of the theories and applications. Earth-Sci Rev 2004;66:199-216.

[49] Merdy P, Huclier S, Koopal LK. Modeling metal-particle interactions with an emphasis on natural organic matter. Environ Sci Technol 2006;40:7459-66.

[50] Groenenberg JE, Lofts S. The use of assemblage models to describe trace element partitioning, speciation, and fate: A review. Environ Toxicol Chem 2014;33:2181-96. 
[51] Tipping E. Humic ion-binding model VI: An improved description of the interactions of protons and

2752

2753

2754

2755

2756

2757

2758

2759

2760

2761

2762

2763

2764

2765

2766

2767

2768

2769

2770

2771

2772

2773

2774

2775

2776

2777

2778

2779

2780

2781

2782

2783

2784

2785

2786

2787 metal ions with humic substances. Aquat Geochem 1998;4:3-47.

[52] Smith E, Rey - Castro C, Longworth H, Lofts S, Lawlor A, Tipping E. Cation binding by acid washed peat, interpreted with humic ion - binding model VI - fd. Eur J Soil Sci 2004;55:433-47.

[53] Tipping E, Lofts S, Sonke J. Humic ion-binding model VII: A revised parameterisation of cation-binding by humic substances. Environ C Chem 2011;8:225-35.

[54] Benedetti MF, Van Riemsdijk WH, Koopal LK. Humic substances considered as a heterogeneous donnan gel phase. Environ Sci Technol 1996;30:1805-13.

[55] Milne CJ, Kinniburgh DG, Tipping E. Generic NICA-donnan model parameters for proton binding by humic substances. Environ Sci Technol 2001;35:2049-59.

[56] Milne CJ, Kinniburgh DG, Van Riemsdijk WH, Tipping E. Generic NICA-donnan model parameters for metal-ion binding by humic substances. Environ Sci Technol 2003;37:958-71.

[57] Koopal LK, Van Riemsdijk WH, De Wit JCM, Benedetti MF. Analytical isotherm equations for multicomponent adsorption to heterogeneous surfaces. J Colloid Interface Sci 1994;166:51-60.

[58] Koopal LK, van Riemsdijk WH, Kinniburgh DG. Humic matter and contaminants. General aspects and modeling metal ion binding. Pure Appl Chem 2001;73:2005-16.

[59] Saito T, Nagasaki S, Tanaka S, Koopal LK. Electrostatic interaction models for ion binding to humic substances. Colloids Surf A 2005;265:104-13.

[60] Zhuravlev L. The surface chemistry of amorphous silica. Zhuravlev model. Colloids Surf A 2000;173:1-38.

[61] Chuang I-S, Maciel GE. Probing hydrogen bonding and the local environment of silanols on silica surfaces via nuclear spin cross polarization dynamics. J Am Chem Soc 1996;118:401-6.

[62] Yang J, Wang E. Reaction of water on silica surfaces. Curr Opin Solid State Mater Sci 2006;10:33-9.

[63] Iler RK. The chemistry of silica: Solubility, polymerization, colloid and surface properties, and biochemistry. New York: Wiley-Interscience; 1979.

[64] Boehm HP. Chapter 5. Advances in catalysis and related subjects, vol 16. Academic Press, New York; 1966. p. 179.

[65] Tamura H, Tanaka A, Mita K, Furuichi R. Surface hydroxyl site densities on metal oxides as a measure for the ion-exchange capacity. J Colloid Interface Sci 1999;209:225-31.

[66] Pauling L. The principles determining the structure of complex ionic crystals. J Am Chem Soc 1929;51:1010-26.

[67] Brown ID. The chemical bond in inorganic chemistry. London: Oxford Univ. Press; 2002.

[68] Brown ID, Altermatt D. Bond-valence parameters obtained from a systematic analysis of the inorganic crystal structure database. Acta Crystallogr Sect B: Struct Sci 1985;41:244-7.

[69] Brown ID. Recent developments in the methods and applications of the bond valence model. Chem Rev 2009;109:6858-919.

[70] Hiemstra T, Van Riemsdijk WH, Bolt GH. Multisite proton adsorption modeling at the solid/solution interface of (hydr) oxides: A new approach: I. Model description and evaluation of intrinsic reaction constants. J Colloid Interface Sci 1989;133:91-104. 
2788

2789

2790

2791

2792

2793

2794

2795

2796

2797

2798

2799

2800

2801

2802

2803

2804

2805

2806

2807

2808

2809

2810

2811

2812

2813

2814

2815

2816

2817

2818

2819

2820

2821

2822

2823

2824

2825

2826

[71] Hiemstra T, Venema P, Van Riemsdijk WH. Intrinsic proton affinity of reactive surface groups of metal (hydr) oxides: The bond valence principle. J Colloid Interface Sci 1996;184:680-92.

[72] Hiemstra T, Vanriemsdijk WH, Bruggenwert MGM. Proton adsorption mechanism at the gibbsite and aluminum-oxide solid-solution interface. Neth J Agric Sci 1987;35:281-93.

[73] Hiemstra T, Van Riemsdijk WH. Effect of different crystal faces on experimental interaction force and aggregation of hematite. Langmuir 1999;15:8045-51.

[74] Hiemstra T, van Riemsdijk WH. On the relationship between surface structure and ion complexation of oxide-solution interface. In: Hubbard AT, (ed). Encyclopedia of surface and colloid science. New York: ; Marcel Dekker; 2002. p. 3773-99.

[75] Machesky ML, Wesolowski DJ, Palmer DA, Ridley MK. On the temperature dependence of intrinsic surface protonation equilibrium constants: An extension of the revised MUSIC model. J Colloid Interface Sci 2001;239:314-27.

[76] Koopal LK. Mineral hydroxides: From homogeneous to heterogeneous modelling. Electrochim Acta 1996;41:2293-305.

[77] Koopal LK. Ion adsorption on mineral oxide surfaces. In: Da̧browski A, Tertykh VA, (eds). Adsorption on new and modified inorganic sorbents. Studies in surface science and catalysis Vol. 99. Amsterdam: Elsevier; 1996. Chapter 3, 5. p. 757 -96.

[78] Hiemstra T, Van Riemsdijk WH. Physical chemical interpretation of primary charging behaviour of metal (hydr) oxides. Colloids Surf 1991;59:7-25.

[79] Hiemenz PC. Principles of colloid and surface chemistry, 2nd edition, revised and expanded. New York: Marcel Dekker; 1986.

[80] Hiemstra T, Van Riemsdijk WH. On the relationship between charge distribution, surface hydration, and the structure of the interface of metal hydroxides. J Colloid Interface Sci 2006;301:1-18.

[81] Hiemstra T, Yong H, Van Riemsdijk WH. Interfacial charging phenomena of aluminum (hydr) oxides. Langmuir 1999;15:5942-55.

[82] Abendroth R. Behavior of a pyrogenic silica in simple electrolytes. J Colloid Interface Sci 1970;34:591-6.

[83] Gibb AW, Koopal LK. Electrochemistry of a model for patchwise heterogeneous surfaces: The rutile-hematite system. J Colloid Interface Sci 1990;134:122-38.

[84] Hiemstra T, Van Riemsdijk WH. A surface structural approach to ion adsorption: The charge distribution (CD) model. J Colloid Interface Sci 1996;179:488-508.

[85] Rietra RP, Hiemstra T, van Riemsdijk WH. The relationship between molecular structure and ion adsorption on variable charge minerals. Geochim Cosmochim Acta 1999;63:3009-15.

[86] Schulze DG. An introduction to soil mineralogy. In: Dixon JB, Schulze DG, (eds). Soil mineralogy with environmental application. Sssa book series no. 7 Vol. Madison: Soil Science Society of America 2002. Chapter 1. p. 1-34.

[87] Johnston CT, Tombacz E. Surface chemistry of soil minerals. In: Dixon JB, Schuze DG, (eds). Soil mineralogy with environmental application. Sssa book series no. 7 Vol. Madison: Soil Science Society of America; 2002. Chapter 2. p. 37-67. 
[88] Bourg IC, Sposito G, Bourg AC. Modeling the acid-base surface chemistry of montmorillonite. J Colloid Interface Sci 2007;312:297-310.

9] Liu X, Lu X, Meijer EJ, Wang R, Zhou H. Atomic-scale structures of interfaces between phyllosilicate edges and water. Geochim Cosmochim Acta 2012;81:56-68.

[90] Liu X, Lu X, Sprik M, Cheng J, Meijer EJ, Wang R. Acidity of edge surface sites of montmorillonite and kaolinite. Geochim Cosmochim Acta 2013;117:180-90.

[91] Koopal LK, van Riemsdijk WH. Electrosorption on random and patchwise heterogeneous surfaces: Electrical double-layer effects. J Colloid Interface Sci 1989;128:188-200.

[92] Avena MJ, De Pauli CP. Proton adsorption and electrokinetics of an argentinean montmorillonite. J Colloid Interface Sci 1998;202:195-204.

[93] Tombacz E, Regdon I. Humic substances as various colloidal systems. In: Senesi N, Miano TM, (eds). Humic substances in the global environment and implications on human health. Amsterdam: Elsevier; 1994. p. 139-44.

[94] Tombácz E, Varga K, Szántó F. An X-ray diffraction study of alkylammonium humate complexes. Colloid Polym Sci 1988;266:734-8.

[95] Traina SJ, McAvoy DC, Versteeg DJ. Association of linear alkylbenzenesulfonates with dissolved humic substances and its effect on bioavailability. Environ Sci Technol 1996;30:1300-9.

[96] Otto WH, Britten DJ, Larive CK. NMR diffusion analysis of surfactant-humic substance interactions. J Colloid Interface Sci 2003;261:508-13.

[97] Koopal LK, Goloub TP, Davis TA. Binding of ionic surfactants to purified humic acid. J Colloid Interface Sci 2004;275:360-7.

[98] Yee MM, Miyajima T, Takisawa N. Evaluation of amphiphilic properties of fulvic acid and humic acid by alkylpyridinium binding study. Colloids Surf A 2006;272:182-8.

[99] Yee MM, Miyajima T, Takisawa N. Thermodynamic studies of dodecylpyridinium ion binding to fulvic acid. Colloids Surf A 2006;287:68-74.

[100] Yee MM, Miyajima T, Takisawa N. Study of ionic surfactants binding to humic acid and fulvic acid by potentiometric titration and dynamic light scattering. Colloids Surf A 2009;347:128-32.

[101] Yee MM, Miyajima T, Takisawa N. Thermodynamic studies of dodecylpyridinium ion binding to humic acid and effect of solution parameters on their binding. Colloids Surf A 2007;295:61-6.

[102] Matsuda M, Kaminaga A, Hayakawa K, Takisawa N, Miyajima T. Surfactant binding by humic acids in the presence of divalent metal salts. Colloids Surf A 2009;347:45-9.

[103] Ishiguro M, Tan WF, Koopal LK. Binding of cationic surfactants to humic substances. Colloids Surf A 2007;306:29-39.

[104] Thieme J, Niemeyer J. Interaction of colloidal soil particles, humic substances and cationic detergents studied by X-ray microscopy. In: Rehage H, Peschel G, (eds). Structure, dynamics and properties of disperse colloidal systems. Progress in colloid \& polymer science Vol.: Steinkopff; Prog. Colloid Polym. Sci. 111; 1998. Chapter 33. p. 193-201.

[105] Barron W, Murray BS, Scales PJ, Healy TW, Dixon DR, Pascoe M. The streaming current detector - a comparison with conventional electrokinetic techniques. Colloids Surf A 1994;88:129-39. 
[106] Walker CA, Kirby JT, Dentel SK. The streaming current detector: A quantitative model. J Colloid Interface Sci 1996;182:71-81.

[107] Kam S-K, Gregory J. The interaction of humic substances with cationic polyelectrolytes. Water Res 2001;35:3557-66.

[108] Tan W-F, Norde W, Koopal LK. Humic substance charge determination by titration with a flexible cationic polyelectrolyte. Geochim Cosmochim Acta 2011;75:5749-61.

[109] Dobias B. Surfactant adsorption and dispersion stability in mineral flotation. In: Dobias B, (ed). Coagulation and flocculation: Theory and applications Surfactant science series Vol. 126. 2nd ed. New York: Taylor and Francis (CRC Press) Boca Raton (USA); 2005. Chapter 10. p. 663-765.

[110] Shang C, Rice JA. Investigation of humate-cetyltrimethylammonium complexes by small-angle X-ray scattering. J Colloid Interface Sci 2007;305:57-61.

[111] Subbiah D, Mishra AK. Humic acid-cetyltrimethylammonium bromide interaction: A fluorimetric study. Luminescence 2009;24:84-9.

[112] Ishiguro M, Koopal LK. Binding of alkylpyridinium chloride surfactants to sodium polystyrene sulfonate. Colloids Surf A 2009;347:69-75.

[113] Chen Y, Droge ST, Hermens JL. Analyzing freely dissolved concentrations of cationic surfactant utilizing ion-exchange capability of polyacrylate coated solid-phase microextraction fibers. J Chromatogr A 2012;1252:15-22.

[114] Chen Y, Hermens JL, Droge ST. Influence of organic matter type and medium composition on the sorption affinity of c12-benzalkonium cation. Environ Pollut 2013;179:153-9.

[115] Ishiguro M, Koopal LK. Predictive model of cationic surfactant binding to humic substances. Colloids Surf A 2011;379:70-8.

[116] Droge S, Goss K-U. Effect of sodium and calcium cations on the ion-exchange affinity of organic cations for soil organic matter. Environ Sci Technol 2012;46:5894-901.

[117] Somasundaran P, Fuerstenau D. Mechanisms of alkyl sulfonate adsorption at the alumina-water interface1. J Phys Chem 1966;70:90-6.

[118] Koopal LK, Lee EM, Böhmer MR. Adsorption of cationic and anionic surfactants on charged metal-oxide surfaces. J Colloid Interface Sci 1995;170:85-97.

[119] Manne S, Cleveland J, Gaub H, Stucky G, Hansma P. Direct visualization of surfactant hemimicelles by force microscopy of the electrical double layer. Langmuir 1994;10:4409-13.

[120] Hough DB, Rendall HM. Adsorption of ionic surfactants. In: Parfitt CD, Rochester CH, (eds). Adsorption from solution at the solid/liquid interface. London: Academic Press; 1983. p. 247-319.

[121] Clunie JS, Ingram BT. Adsorption non-ionic surfactants. In: Parfitt CD, Rochester CH, (eds). London: Academic Press; 1983. p. 105-52.

[122] Manne S, Gaub HE. Molecular-organization of surfactants at solid-liquid interfaces. Science 1995;270:1480-2.

[123] Manne S, Gaub HE. Force microscopy: Measurement of local interfacial forces and surface stresses. Current Opinion in Colloid \& Interface Science 1997;2:145-52. 
[124] Johnson R, Nagarajan R. Modeling self-assembly of surfactants at solid-liquid interfaces. II. Hydrophilic surfaces. Colloids Surf A 2000;167:21-30.

[125] Johnson RA, Nagarajan R. Modeling self-assembly of surfactants at solid/liquid interfaces. I. Hydrophobic surfaces. Colloids Surf A 2000;167:31-46.

[126] Nagarajan R. Theory of micelle formation. In: Esumi K, Ueno M, (eds). Structure-performance relationships in surfactants Surfactant science series Vol. 112. 2nd ed. New York: Marcel Dekker (CRC Press); 1997. p. 1-109.

[127] Koopal LK, Goloub T, de Keizer A, Sidorova MP. The effect of cationic surfactants on wetting, colloid stability and flotation of silica. Colloids Surf A 1999;151:15-25.

[128] Atkin R, Craig VSJ, Wanless EJ, Biggs S. Mechanism of cationic surfactant adsorption at the solid-aqueous interface. Adv Colloid Interface Sci 2003;103:219-304.

[129] Tiberg F, Brinck J, Grant L. Adsorption and surface-induced self-assembly of surfactants at the solid-aqueous interface. Current opinion in colloid \& interface science 1999;4:411-9.

[130] Zhmud B, Tiberg F. Interfacial dynamics and structure of surfactant layers. Adv Colloid Interface Sci 2005;113:21-42.

[131] Schrödle S, Richmond GL. In situ non-linear spectroscopic approaches to understanding adsorption at mineral-water interfaces. J Phys D: Appl Phys 2008;41:033001 (14pp).

[132] Paria S, Khilar KC. A review on experimental studies of surfactant adsorption at the hydrophilic solid-water interface. Adv Colloid Interface Sci 2004;110:75-95.

[133] Zhang R, Somasundaran P. Advances in adsorption of surfactants and their mixtures at solid/solution interfaces. Adv Colloid Interface Sci 2006;123:213-29.

[134] Böhmer MR, Koopal LK. Adsorption of ionic surfactants on variable-charge surfaces. 1. Charge effects and structure of the adsorbed layer. Langmuir 1992;8:2649-59.

[135] Böhmer MR, Koopal LK. Adsorption of ionic surfactants on variable-charge surfaces. 2. Molecular architecture and structure of the adsorbed layer. Langmuir 1992;8:2660-5.

[136] Lee EM, Koopal LK. Adsorption of cationic and anionic surfactants on metal oxide surfaces: Surface charge adjustment and competition effects. J Colloid Interface Sci 1996;177:478-89.

[137] Goloub TP, Koopal LK. Adsorption of cationic surfactants on silica. Comparison of experiment and theory. Langmuir 1997;13:673-81.

[138] Goloub TP, Koopal LK, Bijsterbosch BH, Sidorova MP. Adsorption of cationic surfactants on silica. Surface charge effects. Langmuir 1996;12:3188-94.

[139] Somasundaran P, Kunjappu JT. Insitu investigation of adsorbed surfactants and polymers on solids in solution. Colloids Surf 1989;37:245-68.

[140] Gebhardt JE, Fuerstenau DW. The effect of trace surfactant impurities on the adsorption of sodium dodecyl sulfonate on hematite. J Colloid Interface Sci 1984;101:278-9.

[141] Bhagat RP. Kinetics of sodium dodecyl benzene sulfonate adsorption on hematite and its interaction with polyacrylamide. Colloid Polym Sci 2001;279:33-8. 
[142] Wangchareansak T, Craig VS, Notley SM. Adsorption isotherms and structure of cationic surfactants adsorbed on mineral oxide surfaces prepared by atomic layer deposition. Langmuir 2013;29:14748-55.

[143] De Keizer A, Böhmer MR, Mehrian T, Koopal LK. Adsorption of organic ions at the solid-electrolyte interface. Interpretation of common intersection points. Colloids Surf 1990;51:339-57.

[144] Nevskaia DM, Guerrero-Ruiz A, Lopez-Gonzalez JD. Adsorption of polyoxyethylenic nonionic and anionic surfactants from aqueous solution: Effects induced by the addition of nacl and $\mathrm{CaCl}$. $\mathrm{J}$ Colloid Interface Sci 1998;205:97-105.

[145] Rupprecht HH. Influence of solvents on adsorption of ionic surfactants on highly dispersed silicas. J Pharm Sci 1972;61:700-2.

[146] Gao YY, Du JH, Gu TR. Hemimicelle formation of cationic surfactants at the silica-gel water interface. J Chem Soc Faraday Trans 1 1987;83:2671-9.

[147] Gu T, Huang Z. Thermodynamics of hemimicellization of cetyltrimethylammonium bromide at the silica-gel water interface. Colloids Surf 1989;40:71-6.

[148] Bijsterbosch BH. Characterization of silica surfaces by adsorption from solution - investigations into mechanism of adsorption of cationic surfactants. J Colloid Interface Sci 1974;47:186-98.

[149] Koopal LK, Goloub TP. Self-assembly of ionic surfactants adsorbed on mineral oxides: Surface charge and salt effects. In: Sharma R, (ed). Surfactant adsorption and surface solubilization acs symposium series 615. 78. Washington: American Chemical Society; 1995. Chapter 6. p. 78-103.

[150] Zhu BY, Gu TR. General isotherm equation for adsorption of surfactants at solid liquid interfaces. J Chem Soc Faraday Trans 1 1989;85:3813-7.

[151] Zhu BY, Gu TR, Zhao XL. General isotherm equation for adsorption of surfactants at solid liquid interfaces .2. Applications. J Chem Soc Faraday Trans 1 1989;85:3819-24.

[152] Böhmer MR, Koopal LK. Association and adsorption of nonionic flexible chain surfactants. Langmuir 1990;6:1478-84.

[153] Böhmer MR, Koopal LK, Janssen R, Lee EM, Thomas RK, Rennie AR. Adsorption of nonionic surfactants on hydrophilic surfaces - an experimental and theoretical-study on association in the adsorbed layer. Langmuir 1992;8:2228-39.

[154] Moudgil BM, Soto H, Somasondaran P. In: Nagaraj DR, Somasundaran P, Moudgil BM, (eds). Reagents in mineral technology. Surfactant science series Vol. New York: M. Dekker (CRC Press); 1988. Chapter 3. p. 79.

[155] Pashley RM, Israelachvili JN. A comparison of surface forces and interfacial properties of mica in purified surfactant solutions. Colloids Surf 1981;2:169-87.

[156] Herder PC. Interactions between mica surfaces in dodecyl-and octylammonium chloride solutions. J Colloid Interface Sci 1990;134:346-56.

[157] Menezes JL, Yan J, Sharma MM. The mechanism of alteration of macroscopic contact angles by the adsorption of surfactants. Colloids Surf 1989;38:365-90. 
[158] Mishra SK. In: Nagaraj DR, Somasundaran P, Moudgil BM, (eds). Reagents in mineral technology. Surfactant science series Vol. New York: M. Dekker(CRC Press); 1987. Chapter 7. p. 195.

[159] Novich BE. Flotation response prediction from interfacial properties. Colloids Surf 1990;46:255-69.

[160] Levitz P. Adsorption of non ionic surfactants at the solid/water interface. Colloids Surf A 2002;205:31-8.

[161] Denoyel R, Rouquerol J. Thermodynamic (including microcalorimetry) study of the adsorption of nonionic and anionic surfactants onto silica, kaolin, and alumina. J Colloid Interface Sci 1991;143:555-72.

[162] Gonzalez G, Travalloni-Louvisse AM. The effect of Triton X-100 and ethanol on the wettability of quartz. Langmuir 1989;5:26-9.

[163] Levitz P, Van Damme H, Keravis D. Fluorescence decay study of the adsorption of nonionic surfactants at the solid-liquid interface. 1 . Structure of the adsorption layer on a hydrophilic solid. J Phys Chem 1984;88:2228-35.

[164] Levitz P, Van Damme H. Fluorescence decay study of the adsorption of nonionic surfactants at the solid-liquid interface. 2. Influence of polar chain length. J Phys Chem 1986;90:1302-10.

[165] Postmus BR, Leermakers FAM, Cohen Stuart MA. Self-consistent field modeling of non-ionic surfactants at the silica-water interface: Incorporating molecular detail. Langmuir 2008;24:3960-9.

[166] Del Hoyo C, Dorado C, Rodríguez-Cruz M, Sánchez-Martín M. Physico-chemical study of selected surfactant-clay mineral systems. J Therm Anal Calorim 2008;94:227-34.

[167] Poirier JE, Cases JM. Anionic surfactant adsorption onto silicate minerals the role of the cations. Colloids Surf 1991;55:333-44.

[168] Siracusa PA, Somasundaran P. Adsorption desorption and hysteresis of sulfonates on kaolinite - $\mathrm{pH}$ effects. J Colloid Interface Sci 1986;114:184-93.

[169] Siracusa PA, Somasundaran P. Mechanism of hysteresis in sulfonate kaolinite adsorption desorption systems - chromatographic-separation of isomers. J Colloid Interface Sci 1987;120:100-9.

[170] Ananthapadmanabhan KP, Somasundaran P. Mechanism for adsorption maximum and hysteresis in a sodium dodecylbenzenesulfonate/kaolinite system. Colloids Surf 1983;7:105-14.

[171] Lagaly G, Dékany I. Adsorption on hydrophobized surfaces: Clusters and self-organization. Adv Colloid Interface Sci 2005;114:189-204.

[172] Bergaya F, Theng BKG, Lagaly G. Modified clays and clay minerals. In: Bergaya F, Lagaly G, (eds). Handbook of clay science. 2nd ed: Elsevier; 2013. p. 261-3.

[173] Lagaly G, Ogawa M, Dekany I. Clay mineral organic interactions. In: Bergaya F, Lagaly G, (eds). Handbook of clay science. 2nd ed: Elsevier; 2013. Chapter 7.3. p. 309-78.

[174] de Paiva LB, Morales AR, Díaz FRV. Organoclays: Properties, preparation and applications. Appl Clay Sci 2008;42:8-24.

[175] Lagaly G. Interaction of alkylamines with different types of layered compounds. Solid State Ionics 1986;22:43-51.

[176] Osman MA, Ploetze M, Skrabal P. Structure and properties of alkylammonium monolayers self-assembled on montmorillonite platelets. J Phys Chem B 2004;108:2580-8. 
3018

3019

3020

3021

3022

3023

3024

3025

3026

3027

3028

3029

3030

3031

3032

3033

3034

3035

3036

3037

3038

3039

3040

3041

3042

3043

3044

3045

3046

3047

3048

3049

3050

3051

3052

3053

3054

3055

3056

[177] Heinz H, Vaia RA, Krishnamoorti R, Farmer BL. Self-assembly of alkylammonium chains on montmorillonite: Effect of chain length, head group structure, and cation exchange capacity. Chem Mater 2007;19:59-68.

[178] Liu $\mathrm{X}$, Lu $\mathrm{X}$, Wang $\mathrm{R}$, Zhou $\mathrm{H}$, $\mathrm{Xu} \mathrm{S}$. Interlayer structure and dynamics of alkylammonium-intercalated smectites with and without water: A molecular dynamics study. Clays Clay Miner 2007;55:554-64.

[179] Mehrian T, De Keizer A, Lyklema J. Effect of temperature on the adsorption of organic cations on charged surfaces. Langmuir 1991;7:3094-8.

[180] Mehrian T, De Keizer A, Korteweg A, Lyklema J. Thermodynamics of adsorption of dodecylpyridinium chloride on Na-kaolinite. Colloids Surf A 1993;73:133-43.

[181] Mehrian T, Dekeizer A, Korteweg AJ, Lyklema J. Thermodynamics of micellization of n-alkylpyridinium chlorides. Colloids Surf A 1993;71:255-67.

[182] Patterson D, Barbe M. Enthalpy-entropy compensation and order in alkane and aqueous systems. J Phys Chem 1976;80:2435-6.

[183] Shinoda K. Iceberg formation and solubility. J Phys Chem 1977;81:1300-2.

[184] Xu S, Boyd SA. Cationic surfactant adsorption by swelling and nonswelling layer silicates. Langmuir 1995;11:2508-14.

[185] Wang J, Han B, Dai M, Yan H, Li Z, Thomas R. Effects of chain length and structure of cationic surfactants on the adsorption onto Na-kaolinite. J Colloid Interface Sci 1999;213:596-601.

[186] Qi L, Liao W, Bi Z. Adsorption of conventional and gemini cationic surfactants in nonswelling and swelling layer silicate. Colloids Surf A 2007;302:568-72.

[187] Li Z, Bowman RS. Counterion effects on the sorption of cationic surfactant and chromate on natural clinoptilolite. Environ Sci Technol 1997;31:2407-12.

[188] Li Z, Gallus L. Surface configuration of sorbed hexadecyltrimethylammonium on kaolinite as indicated by surfactant and counterion sorption, cation desorption, and ftir. Colloids Surf A 2005;264:61-7.

[189] Li Z, Gallus L. Adsorption of dodecyl trimethylammonium and hexadecyl trimethylammonium onto kaolinite_-competitive adsorption and chain length effect. Appl Clay Sci 2007;35:250-7.

[190] Borden D, Giese RF. Baseline studies of the clay minerals society source clays: Cation exchange capacity measurements by the ammonia-electrode method. Clays Clay Miner 2001;49:444-5.

[191] Ammann L. Cation exchange and adsorption on clays and clay minerals. Dissertation: Christian-Albrechts-Universität, Kiel; 2003.

[192] Malek NANN, Ramli NI. Characterization and antibacterial activity of cetylpyridinium bromide (cpb) immobilized on kaolinite with different cpb loadings. Appl Clay Sci 2015;109:8-14.

[193] Tertre E, Castet S, Berger G, Loubet M, Giffaut E. Surface chemistry of kaolinite and Na-montmorillonite in aqueous electrolyte solutions at 25 and 60 c: Experimental and modeling study. Geochim Cosmochim Acta 2006;70:4579-99.

[194] Hanna HS, Somasundaran P. Equilibration of kaolinite in aqueous inorganic and surfactant solutions. J Colloid Interface Sci 1979;70:181-91. 
[195] Siracusa PA, Somasundaran P. The role of mineral dissolution in the adsorption of dodecylbenzenesulfonate on kaolinite and alumina. Colloids Surf 1987;26:55-77.

[196] Torn LH, De Keizer A, Koopal LK, Lyklema J. Mixed adsorption of poly (vinylpyrrolidone) and sodium dodecylbenzenesulfonate on kaolinite. J Colloid Interface Sci 2003;260:1-8.

[197] Torn LH, de Keizer A, Koopal LK, Lyklema J, Blokzijl W. Polymer adsorption on a patchwise heterogeneous surface. In: Lagaly G, (ed). Horizons 2000 - aspects of colloid and interface science at the turn of the millenium. Progress in colloid \& polymer science Vol. 109: Steinkopff; 1998. Chapter 18. p. $153-60$.

[198] Deng Y, Dixon JB, White GN. Bonding mechanisms and conformation of poly (ethylene oxide)-based surfactants in interlayer of smectite. Colloid Polym Sci 2006;284:347-56.

[199] Nevskaia D, Guerrero-Ruız A, López-González JdD. Adsorption of polyoxyethylenic surfactants on quartz, kaolin, and dolomite: A correlation between surfactant structure and solid surface nature. J Colloid Interface Sci 1996;181:571-80.

[200] Tahani A, Van Damme H, Noik C, Levitz P. Adsorption of nonionic surfactants on kaolins. J Colloid Interface Sci 1996;184:469-76.

[201] Yang K, Zhu LZ, Xing BS. Sorption of sodium dodecylbenzene sulfonate by montmorillonite. Environ Pollut 2007;145:571-6.

[202] Pan J, Yang G, Han B, Yan H. Studies on interaction of dodecyltrimethylammonium bromide with Na-and Al-montmorillonite. J Colloid Interface Sci 1997;194:276-80.

[203] Xu SH, Boyd SA. Alternative model for cationic surfactant adsorption by layer silicates. Environ Sci Technol 1995;29:3022-8.

[204] Delgado ÁV, González-Caballero F, Hunter RJ, Koopal LK, Lyklema J. Measurement and interpretation of electrokinetic phenomena. J Colloid Interface Sci 2007;309:194-224.

[205] Yariv S, Cross H. Formation of aqueous solutions and suspensions of hydrophobic colloids. Geochemistry of colloid systems. Springer; 1979. p. 157-206.

[206] Tahani A, Karroua M, Van Damme H, Levitz P, Bergaya F. Adsorption of a cationic surfactant on $\mathrm{Na}$-montmorillonite: Inspection of adsorption layer by X-ray and fluorescence spectroscopies. J Colloid Interface Sci 1999;216:242-9.

[207] Zhu J, Zhu L, Zhu R, Chen B. Microstructure of organo-bentonites in water and the effect of steric hindrance on the uptake of organic compounds. Clays Clay Miner 2008;56:144-54.

[208] Cygan RT. Molecular modeling in mineralogy and geochemistry. Reviews in Mineralogy and Geochemistry 2001;42:1-35.

[209] He HP, Galy J, Gerard J-F. Molecular simulation of the interlayer structure and the mobility of alkyl chains in HDTMA+/montmorillonite hybrids. J Phys Chem B 2005;109:13301-6.

[210] Tambach TJ, Boek ES, Smit B. Molecular order and disorder of surfactants in clay nanocomposites. PCCP 2006;8:2700-2.

[211] Cygan RT, Liang J-J, Kalinichev AG. Molecular models of hydroxide, oxyhydroxide, and clay phases and the development of a general force field. J Phys Chem B 2004;108:1255-66. 
[212] Kumar PP, Kalinichev AG, Kirkpatrick RJ. Hydration, swelling, interlayer structure, and hydrogen citrate-intercalated hydrotalcite. J Phys Chem B 2006;110:3841-4.

[213] Zhou Q, Shen W, Zhu JX, Zhu RL, He HP, Zhou JH, Yuan P. Structure and dynamic properties of water saturated CTMA-montmorillonite: Molecular dynamics simulations. Appl Clay Sci 2014;97-98:62-71.

[214] Lokar WJ, Koopal LK, Leermakers FAM, Ducker WA. Self-consistent field analysis of ionic surfactant adsorption regulation in the aqueous film between two neutral solids. J Phys Chem B 2004;108:3633-43.

[215] Lokar WJ, Koopal LK, Leermakers FAM, Ducker WA. Confinement-induced phase behavior and adsorption regulation of ionic surfactants in the aqueous film between charged solids. J Phys Chem B 2004;108:15033-42.

[216] Koopal LK, Leermakers FAM, Lokar WJ, Ducker WA. Confinement-induced phase transition and hysteresis in colloidal forces for surfactant layers on hydrophobic surfaces. Langmuir 2005;21:10089-95.

[217] Leermakers FAM, Koopal LK, Lokar WJ, Ducker WA. Modeling of confinement-induced phase transitions for surfactant layers on amphiphilic surfaces. Langmuir 2005;21:11534-45.

[218] Rheinländer T, Klumpp E, Schwuger MJ. On the adsorption of hydrophobic pollutants on surfactant/clay complexes: Comparison of the influence of a cationic and a nonionic surfactant. J Dispersion Sci Technol 1998;19:379-98.

[219] Sonon LS, Thompson ML. Sorption of a nonionic polyoxyethylene lauryl ether surfactant by $2: 1$ layer silicates. Clays Clay Miner 2005;53:45-54.

[220] Backhaus WK, Klumpp E, Narres H-D, Schwuger MJ. Adsorption of 2, 4-dichlorophenol on montmorillonite and silica: Influence of nonionic surfactants. J Colloid Interface Sci 2001;242:6-13.

[221] Giles CH, Smith D, Huitson A. A general treatment and classification of the solute adsorption isotherm. I. Theoretical. J Colloid Interface Sci 1974;47:755-65.

[222] Lee J-F, Liao P-M, Kuo C-C, Yang H-T, Chiou CT. Influence of a nonionic surfactant (Triton X-100) on contaminant distribution between water and several soil solids. J Colloid Interface Sci 2000;229:445-52.

[223] Yang K, Zhu L, Zhao B. Minimizing losses of nonionic and anionic surfactants to a montmorillonite saturated with calcium using their mixtures. J Colloid Interface Sci 2005;291:59-66.

[224] Koopal LK. The effect of polymer polydispersity on the adsorption isotherm. J Colloid Interface Sci 1981;83:116-29.

[225] Shen Y-H. Preparations of organobentonite using nonionic surfactants. Chemosphere 2001;44:989-95.

Guégan R, Gautier M, Bény J-M, Muller F. Adsorption of a C10E3 non-ionic surfactant on a Ca-smectite. Clays Clay Miner 2009;57:502-9. 
[228] Guégan R. Intercalation of a nonionic surfactant (C10E3) bilayer into a Na-montmorillonite clay. Langmuir 2010;26:19175-80.

[229] Sips R. On the structure of a catalyst surface. J Chem Phys 1948;16:490-5.

[230] Sips R. On the structure of a catalyst surface. II. J Chem Phys 1950;18:1024-6.

[231] Bolt GH, Bruggenwert MGM. Soil chemistry, A. Basic elements. Amsterdam: Elsevier; 1978.

[232] Koopal LK, Ralston J. Chain-length effects in the adsorption of surfactants at aqueous interfaces comparison of existing adsorption models with a new model. J Colloid Interface Sci 1986;112:362-79.

[233] Koopal LK, Keltjens L. Adsorption of ionic surfactants on charged solids. Adsorption models. Colloids Surf 1986;17:371-88.

[234] Scamehorn JF, Schechter RS, Wade WH. Adsorption of surfactants on mineral oxide surfaces from aqueous-solutions .1. Isomerically pure anionic surfactants. J Colloid Interface Sci 1982;85:463-78.

[235] Drach M, Rudziński W, Narkiewicz-Michalek J. Theoretical modeling of ionic surfactant adsorption on mineral oxide surfaces. J Dispersion Sci Technol 2000;21:683-710.

[236] Lajtar L, Narkiewicz-Michalek J, Rudzinski W, Partyka S. A new theoretical approach to adsorption of ionic surfactants at water/oxide interfaces: Studies of the mechanism of cationic surfactant adsorption. Langmuir 1994;10:3754-64.

[237] Li B, Ruckenstein E. Adsorption of ionic surfactants on charged solid surfaces from aqueous solutions. Langmuir 1996;12:5052-63.

[238] Leermakers FAM, Scheutjens JMHM. Statistical thermodynamics of association colloids. I. Lipid bilayer membranes. J Chem Phys 1988;89:3264-74.

[239] Böhmer MR, Koopal LK, Lyklema J. Micellization of ionic surfactants: Calculations based on a self-consistent field lattice model. J Phys Chem 1991;95:9569-78.

[240] Leermakers FAM. Physical chemistry and soft matter (pcc); to research; to self-consistent field theory and its applications (prof.Dr.Ir. http://www.wageningenur.nl/en/Expertise-Services/Chair-groups.htm

[241] Keizer M, Van Riemsdijk WH. ECOSAT. Department of Environmental Science, Subdepartment Soil Science and Plant Nutrition: Wageningen Agricultural University Wageningen; 1998. http://www.wageningenur.nl/en/Expertise-Services/Chair-groups.htm.

[242] Gustafsson JP. Visual MINTEQ ver. 3.0 / 3.1. KTH Royal Institute of Technology, Sweden, email: gustafjp@kth.se.

[243] Kinniburgh DG, Tang CK. FIT; Technical Report WD/93/23. British Geological Survey, Natural Environment Research Council.: Keyworth, Great Britain; 1993.

[244] Zhu LZ, Yang K, Lou BF, Yuan BH. A multi-component statistic analysis for the influence of sediment/soil composition on the sorption of a nonionic surfactant (Triton X-100) onto natural sediments/soils. Water Res 2003;37:4792-800.

[245] Davis JA. Adsorption of natural dissolved organic matter at the oxide/water interface. Geochim Cosmochim Acta 1982;46:2381-93. 
3172

[246] Varadachari C, Chattopadhyay T, Ghosh K. Complexation of humic substances with oxides of iron

3175

3176

3177

3178

3179

3180

3181

3182

3183

3184

3185

3186

3187

3188

3189

3190

3191

3192

3193

3194

3195

3196

3197

3198

3199

3200

3201

3202

3203

3204

3205

3206

3207

3208

3209 and aluminum. Soil Sci 1997;162:28-34.

[247] Gu B, Schmitt J, Chen Z, Liang L, McCarthy JF. Adsorption and desorption of natural organic matter on iron oxide: Mechanisms and models. Environ Sci Technol 1994;28:38-46.

[248] Vermeer AWP, Van Riemsdijk WH, Koopal LK. Adsorption of humic acid to mineral particles. 1. Specific and electrostatic interactions. Langmuir 1998;14:2810-9.

[249] Saito T, Koopal LK, van Riemsdijk WH, Nagasaki S, Tanaka S. Adsorption of humic acid on goethite: Isotherms, charge adjustments, and potential profiles. Langmuir 2004;20:689-700.

[250] Murti GSR, Volk VV, Jackson ML. Soil adsorption of linear alkylate sulfonate. Soil Sci Soc Am Proc 1966;30:685-\&.

[251] Urano K, Saito M, Murata C. Adsorption of surfactants on sediments. Chemosphere 1984;13:293-300.

[252] Hand VC, Williams GK. Structure-activity-relationships for sorption of linear alkylbenzenesulfonates. Environ Sci Technol 1987;21:370-3.

[253] McAvoy DC, White CE, Moore BL, Rapaport RA. Chemical fate and transport in a domestic septic system - sorption and transport of anionic and cationic surfactants. Environ Toxicol Chem 1994;13:213-21.

[254] Fytianos K, Voudrias E, Papamichali A. Behavior and fate of linear alkylbenzene sulfonate in different soils. Chemosphere 1998;36:2741-6.

[255] Westall JC, Chen H, Zhang WJ, Brownawell BJ. Sorption of linear alkylbenzenesulfonates on sediment materials. Environ Sci Technol 1999;33:3110-8.

[256] Rodriguez-Cruz MS, Sanchez-Martin MJ, Sanchez-Camazano M. A comparative study of adsorption of an anionic and a non-ionic surfactant by soils based on physicochemical and mineralogical properties of soils. Chemosphere 2005;61:56-64.

[257] Somasundaran P, Snell ED, Xu Q. Adsorption behavior of alkylarylethoxylated alcohols on silica. J Colloid Interface Sci 1991;144:165-73.

[258] Ahmed F, Ishiguro M. Effect of adsorption site potential on adsorption of sodium dodecylbenzenesulfonate in highly humic volcanic ash soil. Soil Sci Plant Nutr 2015;61:432-9.

[259] Ahmed F, Ishiguro M, Moriguchi K. Adsorption of sodium dodecylbenzene sulfonate on highly humic non-allophanic andisol at high-electrolyte concentration. J Jpn Soc Soil Phys 2012:45-54.

[260] Inoue K, Kaneko K, Yoshida M. Adsorption of dodecylbenzenesulfonates by soil colloids and influence of soil colloids on their degradation. Soil Sci Plant Nutr 1978;24:91-102.

[261] Dick SG, Fuersten.D.W., Healy TW. Adsorption of alkylbenzene sulfonate (abs) surfactants at alumina-water interface. J Colloid Interface Sci 1971;37:595-\&.

[262] Ishiguro M, Song KC, Yuita K. Ion-transport in an allophanic andisol under the influence of variable charge. Soil Sci Soc Am J 1992;56:1789-93.

[263] Iwata S, Tabuchi T, Warkentin BP. Soil-water interactions: Mechanisms and applications. New York: Marcel Dekker; 1995. 
3210

3211

3212

3213

3214

3215

3216

3217

3218

3219

3220

3221

3222

3223

3224

3225

3226

3227

3228

3229

3230

3231

3232

3233

3234

3235

3236

3237

3238

3239

3240

3241

3242

3243

3244

3245

3246

3247

3248

[264] Ying GG. Fate, behavior and effects of surfactants and their degradation products in the environment. Environ Int 2006;32:417-31.

[265] Atay NZ, Yenigun O, Asutay M. Sorption of anionic surfactants SDS, AOT and cationic surfactant hyamine 1622 on natural soils. Water Air Soil Pollut 2002;136:55-67.

[266] Bera A, Kumar T, Ojha K, Mandal A. Adsorption of surfactants on sand surface in enhanced oil recovery: Isotherms, kinetics and thermodynamic studies. Appl Surf Sci 2013;284:87-99.

[267] Sanchez-Martin MJ, Rodriguez-Cruz MS, Sanchez-Camazano M. Study of the desorption of linuron from soils to water enhanced by the addition of an anionic surfactant to soil-water system. Water Res 2003;37:3110-7.

[268] Deshpande S, Shiau BJ, Wade D, Sabatini DA, Harwell JH. Surfactant selection for enhancing ex situ soil washing. Water Res 1999;33:351-60.

[269] Paria S. Surfactant-enhanced remediation of organic contaminated soil and water. Adv Colloid Interface Sci 2008;138:24-58.

[270] Lee JF, Hsu MH, Lee CK, Chao HP, Chen BH. Effects of soil properties on surfactant adsorption. J Chin Inst Eng 2005;28:375-9.

[271] Li XX, Yang GP, Cao XY. Sorption behaviors of sodium dodecylbenzene sulfonate (sdbs) on marine sediments. Water Air Soil Pollut 2008;194:23-30.

[272] Xu SH, Boyd SA. Cationic surfactant sorption to a vermiculitic subsoil via hydrophobic bonding. Environ Sci Technol 1995;29:312-20.

[273] Paria S, Yuet PK. Effects of chain length and electrolyte on the adsorption of n-alkylpyridinium bromide surfactants at sand-water interfaces. Ind Eng Chem Res 2006;45:712-8.

[274] Ishiguro M, Torigoe T, Kameoka Y, Akae T. Linear anionic surfactant (SDS) transport in high humic volcanic ash soil and its permeability. Irrig Drain Rural Eng J 2008;76:485-91.

[275] Kang S, Jeong HY. Sorption of a nonionic surfactant Tween 80 by minerals and soils. J Hazard Mater 2015;284:143-50.

[276] Koopal LK, Avena MJ. A simple model for adsorption kinetics at charged solid-liquid interfaces. Colloids Surf A 2001;192:93-107.

[277] West CC. Surfactant-enhanced solubilization of tetrachloroethylene and degradation products in pump and treat remediation. ACS Symp Ser 1992;491:149-58.

[278] Rouse JD, Sabatini DA, Harwell JH. Minimizing surfactant losses using twin-head anionic surfactants in subsurface remediation. Environ Sci Technol 1993;27:2072-8.

[279] Allred B, Brown GO. Surfactant-induced reductions in soil hydraulic conductivity. Ground Water Monit Rem 1994;14:174-84.

[280] Liu MW, Roy D. Surfactant-induced interactions and hydraulic conductivity changes in soil. Waste Manage (Oxford) 1995;15:463-70.

[281] Gabr MA, Chen J, Thomas R. Soil clogging during surfactant-enhanced flushing of naphthalene-contaminated sand-kaolinite. Can Geotech J 1998;35:976-85.

[282] Gardner KH, Arias MS. Clay swelling and formation permeability reductions induced by a nonionic surfactant. Environ Sci Technol 2000;34:160-6. 
[283] Tumeo MA. A survey of the causes of surfactant-induced changes in hydraulic conductivity. Ground Water Monit Rem 1997;17:138-44.

[284] Warkentin BP. Soil structure: A history from tilth to habitat. Adv Agron 2008;97:239-72.

[285] Quirk JP. Interparticle forces - a basis for the interpretation of soil physical behavior. Adv Agron 1994;53:121-83.

[286] Low PF. Physical chemistry of clay-water interaction. Adv Agron 1961;13:269-327.

[287] Lagaly G, Ziesmer S. Colloid chemistry of clay minerals: The coagulation of montmorillonite dispersions. Adv Colloid Interface Sci 2003;100:105-28.

[288] Evangelou V. Environmental soil and water chemistry. New York: Wiley Interscience; 1998.

[289] Hillel D. Environmental soil physics: Fundamentals, applications, and environmental considerations: Academic press; 1998.

[290] Ishiguro M. Ion transport and permeability in an allophanic andisol at low pH. Soil Sci Plant Nutr 2005;51:637-40.

[291] Ishiguro M, Nakaishi K, Nakajima T. Saturated hydraulic conductivity of a volcanic ash soil affected by repulsive potential energy in a multivalent anionic system. Colloids Surf A 2003;230:81-8.

[292] Ishiguro M, Nakajima T. Hydraulic conductivity of an allophanic andisol leached with dilute acid solutions. Soil Sci Soc Am J 2000;64:813-8.

[293] Suarez D, Rhoades J, Lavado R, Grieve C. Effect of pH on saturated hydraulic conductivity and soil dispersion. Soil Sci Soc Am J 1984;48:50-5.

[294] Callaghan MV, Cey EE, Bentley LR. Hydraulic conductivity dynamics during salt leaching of a sodic, structured subsoil. Soil Sci Soc Am J 2014;78:1563-74.

[295] McNeal BL, Coleman NT. Effect of solution composition on soil hydraulic conductivity. Soil Sci Soc Am Proc 1966;30:308-12.

[296] McNeal BL, Layfield DA, Norvell WA, Rhoades JD. Factors influencing hydraulic conductivity of soils in presence of mixed-salt solutions. Soil Sci Soc Am Proc 1968;32:187-90.

[297] Shainberg I, Rhoades J, Prather R. Effect of low electrolyte concentration on clay dispersion and hydraulic conductivity of a sodic soil. Soil Sci Soc Am J 1981;45:273-7.

[298] Mustafa M, Letey J. The effect of two nonionic surfactants on aggregate stability of soils. Soil Sci 1969;107:343-7.

[299] Ishiguro M, Fujii T. Upward infiltration into porous media as affected by wettability and anionic surfactants. Soil Sci Soc Am J 2008;72:741-9. 
Figure captions

3283

Fig. 1. Some examples of synthetic and natural surfactants.

3285

3286

3287

3288

3289

3290

3291

3292

3293

3294

3295

3296

3297

3298

3299

3300

3301

3302

3303

3304

3305

3306

3307

Fig. 2. Schematic illustration of the effect of surfactants on the surface tension of dilute aqueous solutions (a, c, d) and the adsorption isotherm (b) that can be obtained from figure (a) through application of the Gibbs equation. The kink in the surface tension plots (a, c, d) is the CMC.

Fig. 3. Set of surface tension vs. $\ln c$ curves for aqueous solutions of sodium dodecyl pyridinium chloride $(D P C)$ in the presence of different concentrations of $\mathrm{NaCl}(\bullet 0$ $\mathrm{mmol} / \mathrm{L}$; $\square 5 \mathrm{mmol} / \mathrm{L}$; o $20 \mathrm{mmol} / \mathrm{L} ; \Delta 100 \mathrm{mmol} / \mathrm{L})$.

Fig. 4. Example of proton binding to a humic acid at four $\mathrm{KCl}$ concentrations. Data from ref. [45].

Fig. 5. Comparison of the charge density curves vs. $\mathrm{pH}$ and ionic strength of gibbsite, silica and hematite. The ionic strength values of the curves of hematite are $(\Delta) 0.002 \mathrm{M}$, (X) $0.01 \mathrm{M}$, and (O) $0.1 \mathrm{M}$. Data from ref. [81-83].

Fig. 6. Proton charge density of the montmorillonite for three values of the 1-1 electrolyte concentration (symbols) and model predictions (curves). Data from ref. [92].

Fig. 7. Binding of C12PC and C16PC to PAHA (mmol surfactant/g PAHA) at I $=0.025 \mathrm{M}$ and (a) $\mathrm{pH}$ 5, (b) $\mathrm{pH} 7$, and (c) $\mathrm{pH} 10$. The reduced monomer concentration, $\mathrm{c} / \mathrm{CMC}$, normalizes the concentration differences betwe en C12PC and C16PC. The arrows indicate the amount of negative charge (mmol/g) of PAHA in $0.02 \mathrm{M} \mathrm{KNO}_{3}$ at the given $\mathrm{pH}$ values. Data from ref. [97].

Fig. 8. Binding isotherms for $C 12 \mathrm{P}^{+}-\mathrm{AFA}$ system as a function of $\mathrm{pH}$ at: (a) $\mathrm{I}=0.03 \mathrm{~mol}$

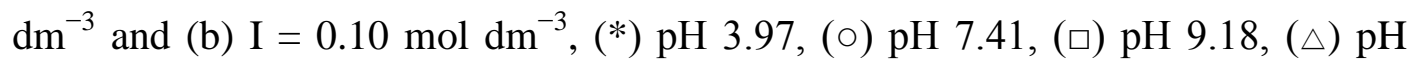
10.01. Data from ref. [99].

Fig. 9. Binding isotherms for (a) AHA system and (b) AFA system. Data from ref. [98].

Fig. 10. Binding isotherms of $\mathrm{C}_{16} \mathrm{P}^{+}$to humic acid (PAHA) at two ionic strengths, (a) 
log-normal plot with $\mathrm{H}^{+}$release, (b) double logarithmic plot, and binding isotherms of $\mathrm{C} 12 \mathrm{P}^{+}$to anionic polyelectrolyte (SPSS) at three ionic strengths, (c) log-normal plot, (d) double logarithmic plot. Data from ref.[103, 112].

Fig. 11. Influence of cationic surfactant carbon-chain length on binding to purified Aldrich

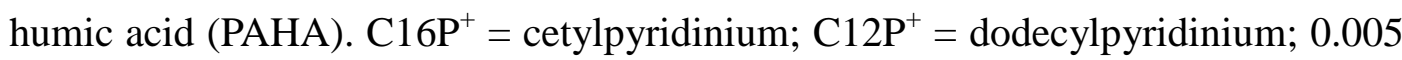
$\mathrm{M} \mathrm{NaCl}$. (a) log-normal plot, (b) double logarithmic plot. Data from ref. [103].

Fig. 12. Binding of cationic surfactant (dodecylpyridinium chloride, C12PC) to various humic and fulvic acids at $0.005 \mathrm{M} \mathrm{NaCl}$ and pH 5. PAHA, Aldrich humic acid; IHA, Inogashira humic acid; DHA, Dando humic acid; SFA, Strichen fulvic acid; LFA, Laurentian fulvic acid. (a) log-normal plot, (b) double logarithmic plot. Data from ref.[103].

Fig. 13. Benzyldimethyldodecyl-ammonium chloride (C12-BAC) sorption to purified Aldrich humic acid (AHA) determined at different background electrolytes at $\mathrm{pH} 6$ (A) and at different $\mathrm{pH}$ in the presence of $5 \mathrm{mM} \mathrm{NaCl}$ or $\mathrm{CaCl}_{2}$ (B). The isotherms were fitted with Freundlich equation with Freundlich exponent $\left(n_{\mathrm{F}}\right)$ of 0.8. Data from ref. [114].

Fig. 14. Adsorption isotherms of SNBS (sodium C9-benzene sulfonate; $\mathrm{pH}$ 4.1) and DPC (C12-pyridinium chloride; pH 8.0) on rutile at $0.01 \mathrm{M} \mathrm{NaCl}$. Panel (a) is a double logarithmic representation of the isotherms and depicts the typical four-regions isotherms; panel (b) depicts the same results in a semi-logarithmic plot. Data from ref. [118].

Fig. 15. Adsorption isotherms of sodium nonyl-benzene-sulfonate (SNBS) on rutile at pH 4.1 and three salt concentrations are depicted as log-log (a) and lin-log (b) plots. Data from ref.[134].

Fig. 16. Adsorption of SNBS and the net proton adsorption, $\Gamma(0)$, on rutile as a function of the equilibrium SNBS concentration at three $\mathrm{pH}$ values and two $\mathrm{NaCl}$ concentrations; the $\mathrm{pH}_{\mathrm{pznpc}}$ of rutile is 5.85. Data from ref. [134].

Fig. 17. Adsorption isotherms for C12PC on Aerosil, measured at $\mathrm{pH} 9$ and two salt concentrations (a) as log-log plot and (b) as lin-log plot. Data from ref. [137].

Fig. 18. Effect of DPC adsorption on the surface charge of Aerosil, $\Gamma(0)$, at $\mathrm{pH} 9$. Data from ref. [138]. 
3340

3341

3342

3343

3344

3345

3346

3347

3348

3349

3350

3351

3352

3353

3354

3355

3356

3357

3358

3359

3360

3361

3362

3363

3364

3365

3366

3367

3368

3369

3370

3371

3372

Fig. 19. Adsorption of tertiair-octylbenzene polyoxyethylene glycol surfactants on Spherosil silica at $25{ }^{\circ} \mathrm{C}$ and $\mathrm{pH}$ 6.5. The TBE surfactants are monodisperse $[\mathrm{TBE}=$ $\left.\mathrm{C} 8 \Phi(\mathrm{EO})_{\mathrm{Np}}\right]$, the $\mathrm{TX}$ surfactants are polydisperse $\left[\mathrm{TX}=\mathrm{C} 8 \Phi(\mathrm{EO})_{<\mathrm{Np}}\right] \mathrm{Np}$ is indicated in the figure. Data from ref. [160].

Fig. 20. Adsorption of $\mathrm{C} 12 \mathrm{PC}$ on sodium kaolinite as a function of the $\mathrm{NaCl}$ concentration at $\mathrm{pH} 5$ and $20^{\circ} \mathrm{C}$; the horizontal line indicates the isoelectric situation and the axis on the right side indicates the sign of the electrophoretic mobility. Data from ref. [179].

Fig. 21. Adsorption isotherms of C16TAC (=HDTMA) on kaolinite at pH 6.5 and two $\mathrm{NaCl}$ concentrations. The adsorption is expressed relative to the CEC at $\mathrm{pH} 6.5$, which is $40 \mu \mathrm{mol} / \mathrm{g}$. Data from ref. [184]

Fig. 22. Abstraction (adsorption + precipitation) isotherms of sodium dodecylbenzene sulfonate (SDBS) - kaolinite system at different $\mathrm{pH}$ (indicated), $0.1 \mathrm{M} \mathrm{NaCl}$ and 25 ${ }^{\circ} \mathrm{C}$. Data from ref. [195].

Fig. 23. Adsorption isotherms of three nonionic surfactants on kaolinite at $\mathrm{pH} 3.8$ and $\mathrm{T}=25^{\circ} \mathrm{C}$. The three surfactants are polydisperse alkylbenzene polyoxyethelenes form the Triton series: $\mathrm{TX} 100=\mathrm{C}_{8} \mathrm{H}_{17} \Phi E \mathrm{O}_{9.5}, \mathrm{TX} 165=\mathrm{C}_{8} \mathrm{H}_{17} \Phi \mathrm{EO}_{16}$ and $\mathrm{TX} 305=$ $\mathrm{C}_{8} \mathrm{H}_{17} \Phi \mathrm{EO}_{30}$. Data from ref. [161].

Fig. 24. Characteristic C16TAC (=HDTMA) adsorption isotherms on Na- and Ca-saturated montmorillonite (Wyoming montmorillonite, SWy-1; CEC $=900 \mu \mathrm{mol} / \mathrm{g}$ ), compared with that on Na-kaolinite (Source Clays Repository, Dept. of Geology, University of Missouri, Columbia; CEC $=40 \mu \mathrm{mol} / \mathrm{g}$ at $\mathrm{pH}$ 6.5). The adsorbed amount is expressed relative to the CEC, the concentration of C16TAC (=HDTMA) is in mol/L. The clays were free of organic matter and homo-ionic (washing first with a $\mathrm{NaCl}$ solution and for Ca-clays subsequently with a $\mathrm{CaCl}_{2}$ solution). Data from ref. [203].

Fig. 25. (a) Adsorption of C16TAC (=HDTMA) on Na-SWy-1 in $5 \mathrm{mmol} / \mathrm{L} \mathrm{NaCl}$ solution and the concomitant Na release. (b) Electrophoretic mobility (solid symbols) and degree of dispersion stability (open symbols) of C16TAC-Na-SWy-1 as a function of the C16TAC adsorption; (c) d(001)-spacings of C16TAC-Na-SWy-1 obtained by X-ray diffraction as a function of C16TAC adsorption at three different water contents (dried at different relative humidity, the $\mathrm{RH}$ values are indicated). The C16TAC adsorption is expressed relative to the CEC (900 $\mu \mathrm{mol} / \mathrm{g})$. Data from ref. [184]. 
3373

3374

3375

3376

3377

3378

3379

3380

3381

3382

3383

3384

3385

3386

3387

3388

3389

3390

3391

3392

3393

3394

3395

3396

3397

3398

3399

3400

3401

3402

Fig. 26. Calculated density distributions for ammonium $\mathrm{N}$, alkyl $\mathrm{C}, \mathrm{Ca}^{2+}, \mathrm{Br}^{-}$and water $\mathrm{O}$ for montmorillonite (Mt) with a CEC of $1060 \mu \mathrm{mol}_{\mathrm{e}} / \mathrm{g}$ and different loading levels of $\mathrm{C} 16 \mathrm{TA}^{+} / \mathrm{C} 16 \mathrm{TAB}$ relative to the $\mathrm{CEC}$; the loadings are indicated in the figure. Data from ref. [213].

Fig. 27. Snapshots of the surfactant configurations in the Mt-C16TA+/C16TAB system. The panels a, b, c and d correspond with, respectively, Figs. 25a, 25b, 25c, and 25d. The stick layers represents the Mt-layers, the atoms in the interlayer are colored: $\mathrm{Ca}=$ green, $\mathrm{N}$ = blue, $\mathrm{C}$ = grey, $\mathrm{Br}=$ brown, $\mathrm{H}=$ white, $\mathrm{O}$ water = red. Data from ref. [213].

Fig. 28. Adsorption isotherms of C12(EO)8 on different layer silicates: ( $\boldsymbol{\nabla}$ ) Na-kaolinite, $(\boldsymbol{\square})$ Na-illite, $(\boldsymbol{\bullet})$ Na-montmorillonite $(\mathrm{Na}-\mathrm{Mt})$ and $(\boldsymbol{\Delta})$ Ca-bentonite (Ca-Mt). Data adapted from ref. [218].

Fig. 29. Effect of carbon chain length on linear alkylbenzenesulfonate (LAS) adsorption in a sediment. Data from ref. [255].

Fig. 30. Adsorption isotherms of cationic (C16TAB), nonionic (Tergitol 15-S-7 = C11-15(EO)7; secondary alcohol ethoxylate) and anionic (SDS) surfactants for sand. Data from ref. [266]

Fig. 31. Influence of $\mathrm{pH}$ on cationic (C16TAB), nonionic (Tergitol 15-S-7= C11-15(EO)7) and anionic (SDS) surfactant adsorption on sand. Data from ref. [266]

Fig. 32. (a) Influence of electrolyte concentration on the adsorption of the anionic surfactant, sodium dodecylbenzenesulfonate on highly humic non-allophanic andosol. The $\mathrm{NaCl}$ concentrations are indicated in the figure. Symbols are measured values. Solid lines are calculated with the Langmuir-Donnan model. (b) Donnan potentials obtained with the Langmuir-Donnan model. Data from ref. [258].

Fig. 33. Sodium dodecylsulfate (SDS) adsorption and degradation in a soil suspension (highly humic nonallophanic andosol) in relation to mixing time. Data from ref. [274].

Fig. 34. The adsorption of hexadecyltrimethylammonium (HDTMA $=\mathrm{C}_{16 \mathrm{TA}}{ }^{+}$) on two vermiculitic soils ( $\mathrm{Na}$ and $\mathrm{Ca}$ version) and the corresponding electrophoretic mobilities and optical densities of the soil suspensions. (a) Adsorption isotherms, 
(b) electrophoretic mobilities of the soils and (c) optical densities of the soils. Data from [272].

3405 Fig. 35. Soil hydraulic conductivity during percolation with a $1 \mathrm{~mol} / \mathrm{kg}$ surfactant solution (A) nonionic surfactants (N1, N2, N3), (B) anionic surfactants (A1, A2), cationic surfactants (C1, C2) and amphoteric surfactant (Am). Data from ref. [279].

3408 Fig. 36. Comparison of upward infiltration of sodium dodecylsulfate (SDS) solution at 70 $\mathrm{mmol} / \mathrm{L}$ with that of pure water in a peat moss column. The water table connected with the column was set at height $0 \mathrm{~cm}$. Data from ref. [299] 

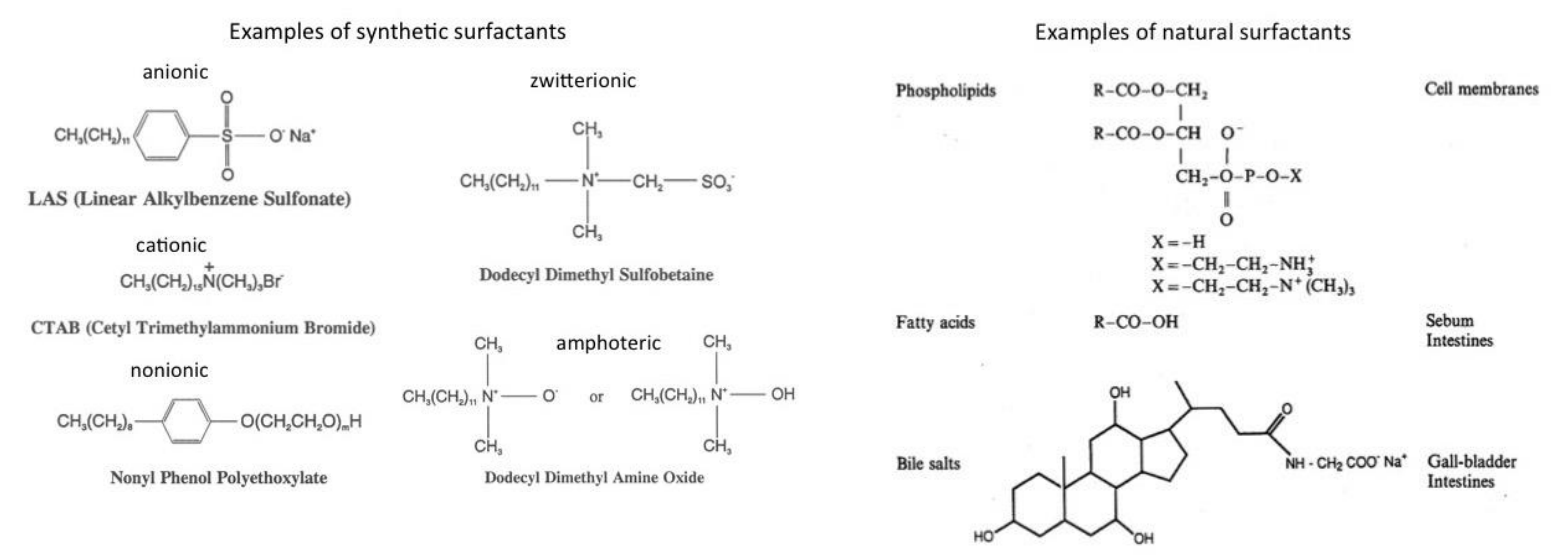

Fig. 1. 

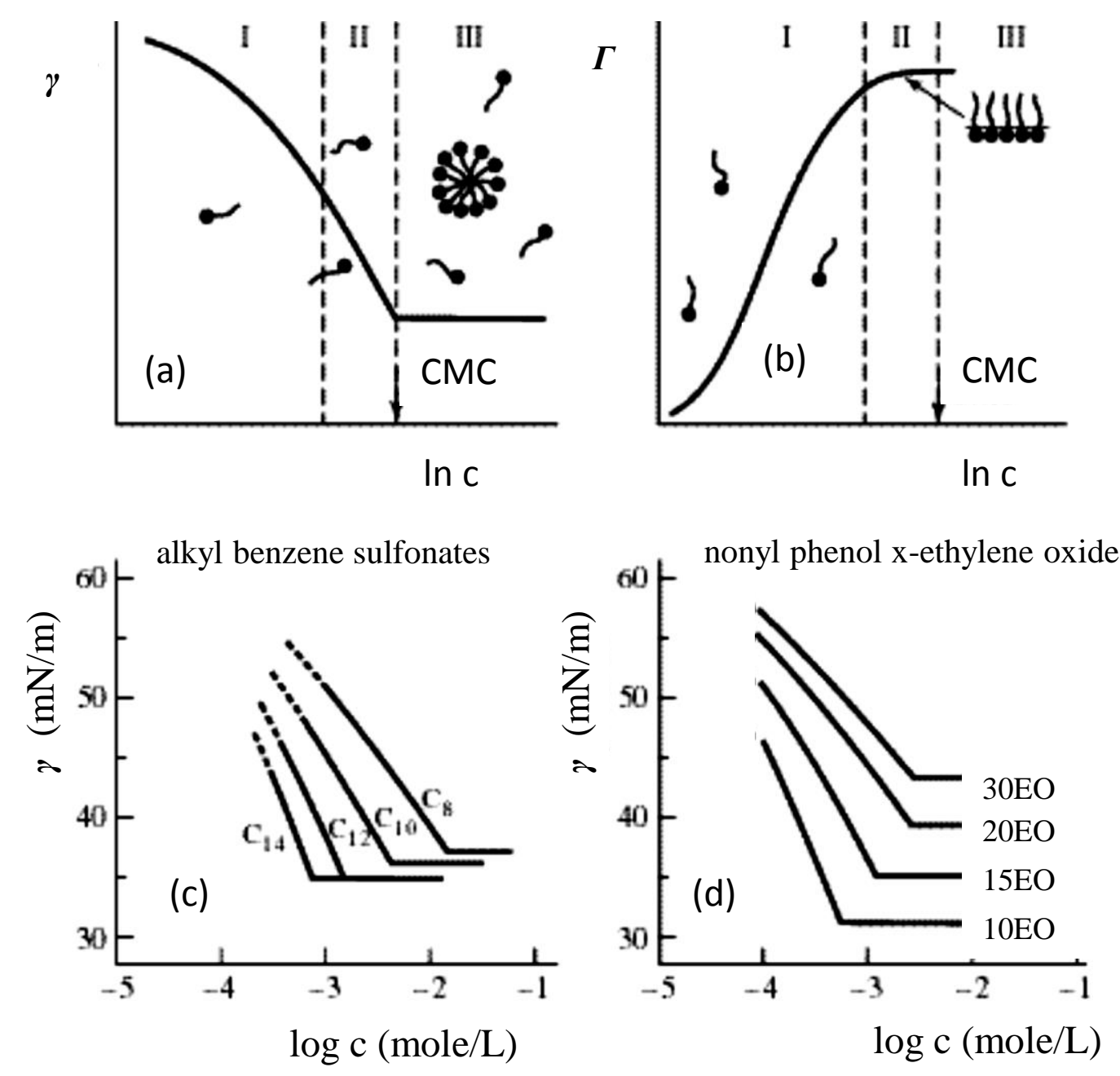

Fig. 2. 


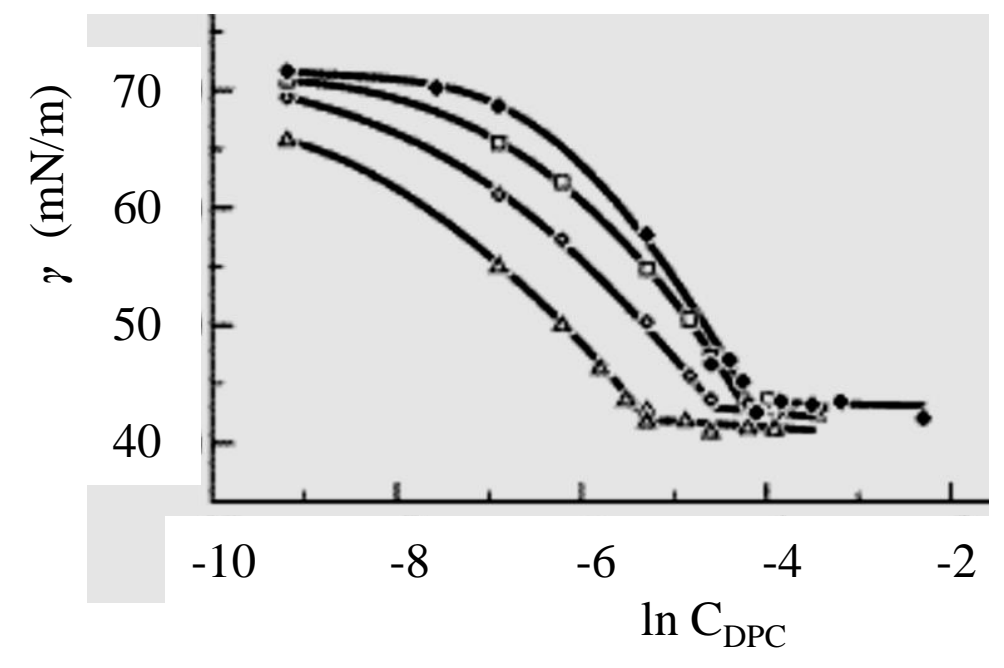

Fig. 3. 


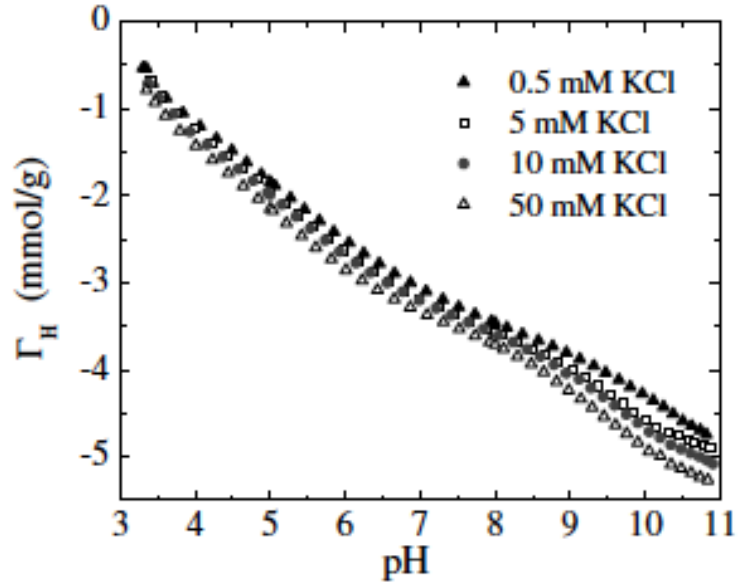

Fig. 4. 

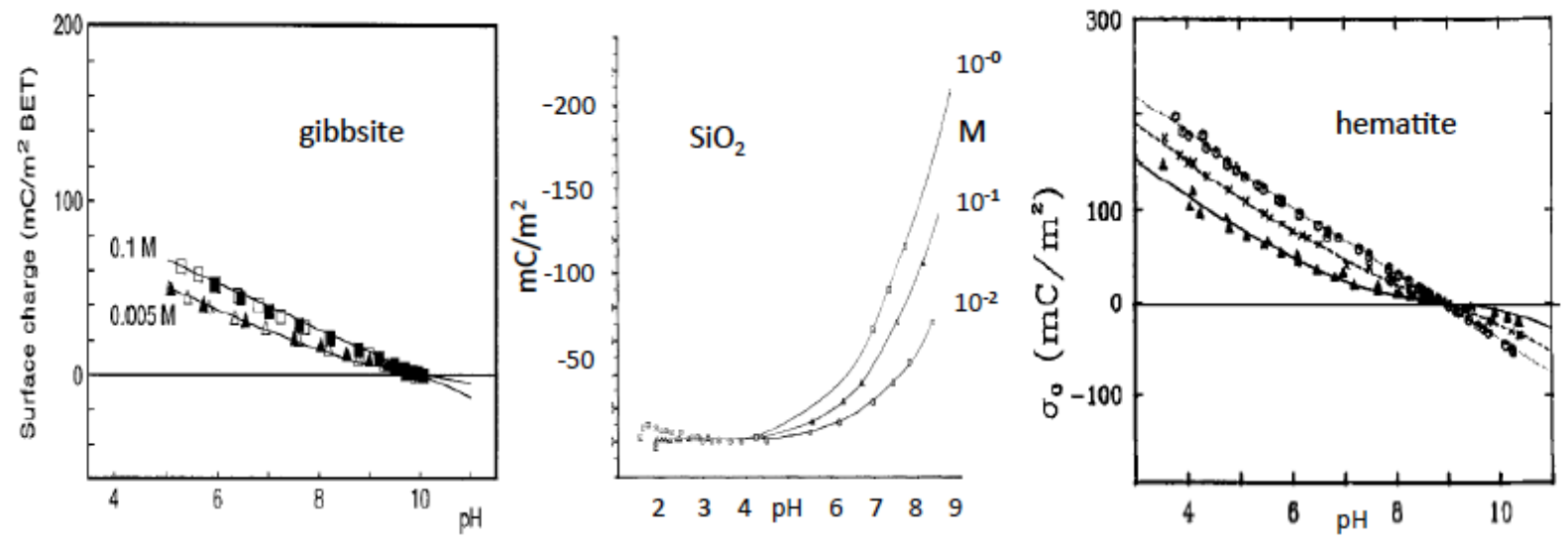

Fig. 5. 


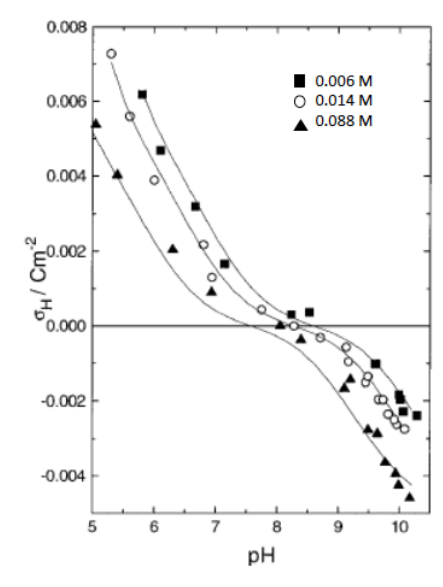

Fig. 6. 


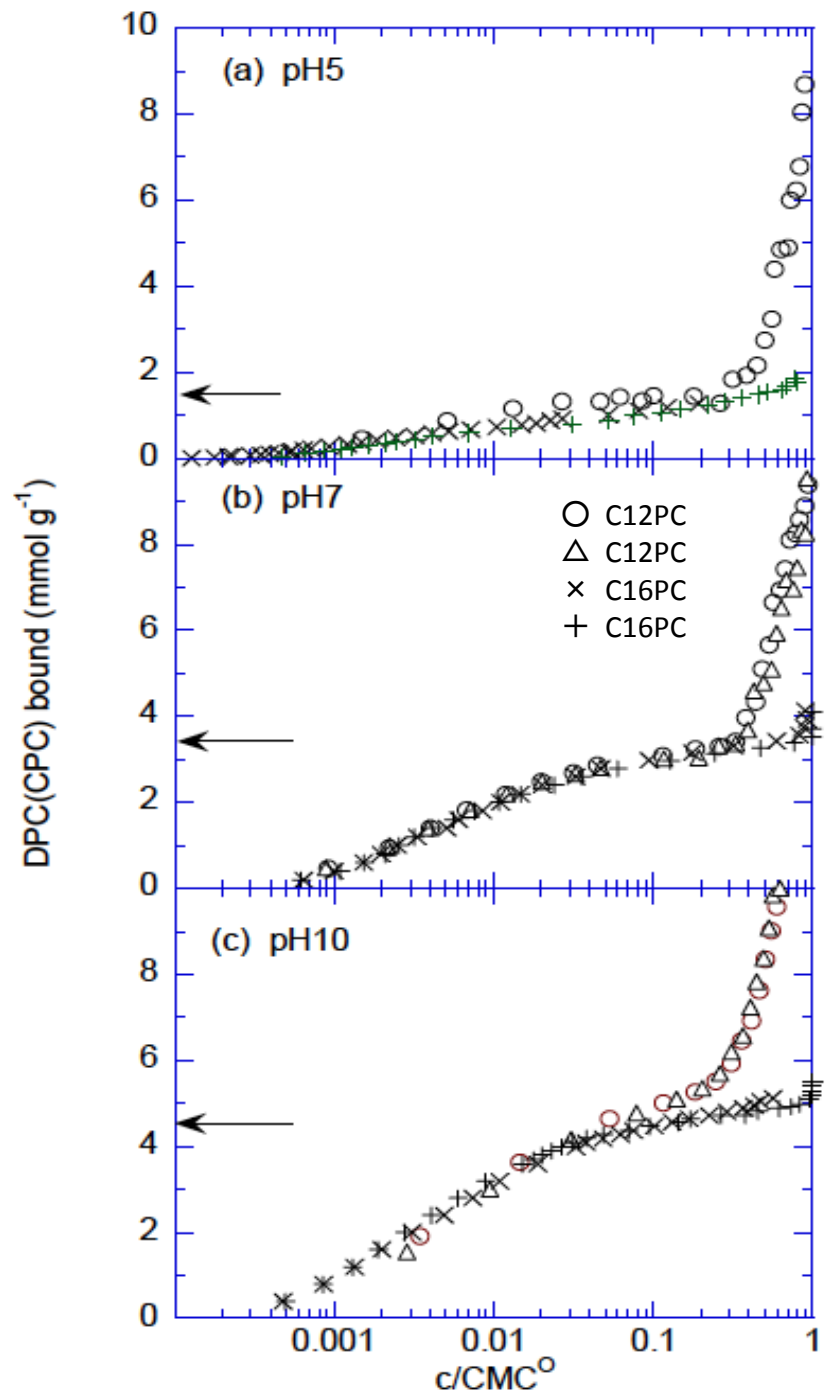

Fig. 7. 

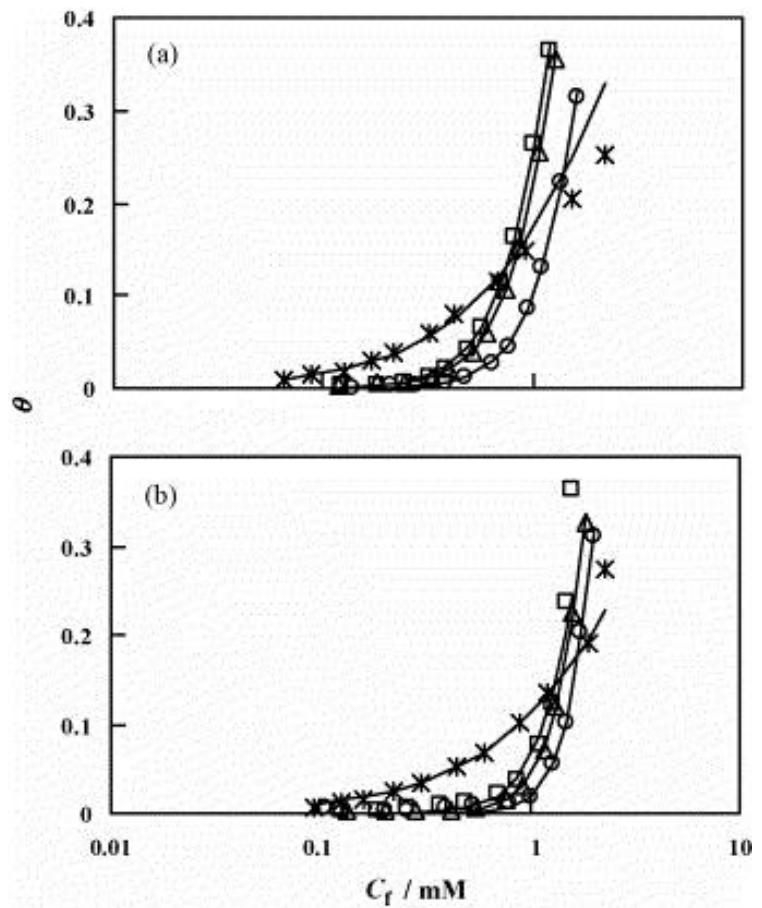

Fig. 8. 


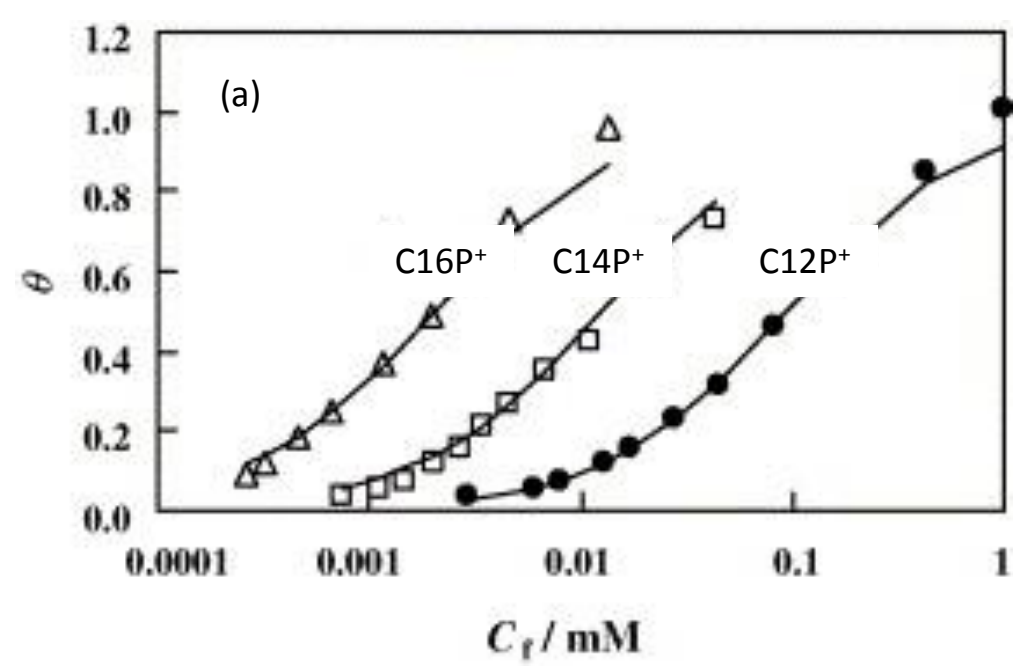

Fig. 9.

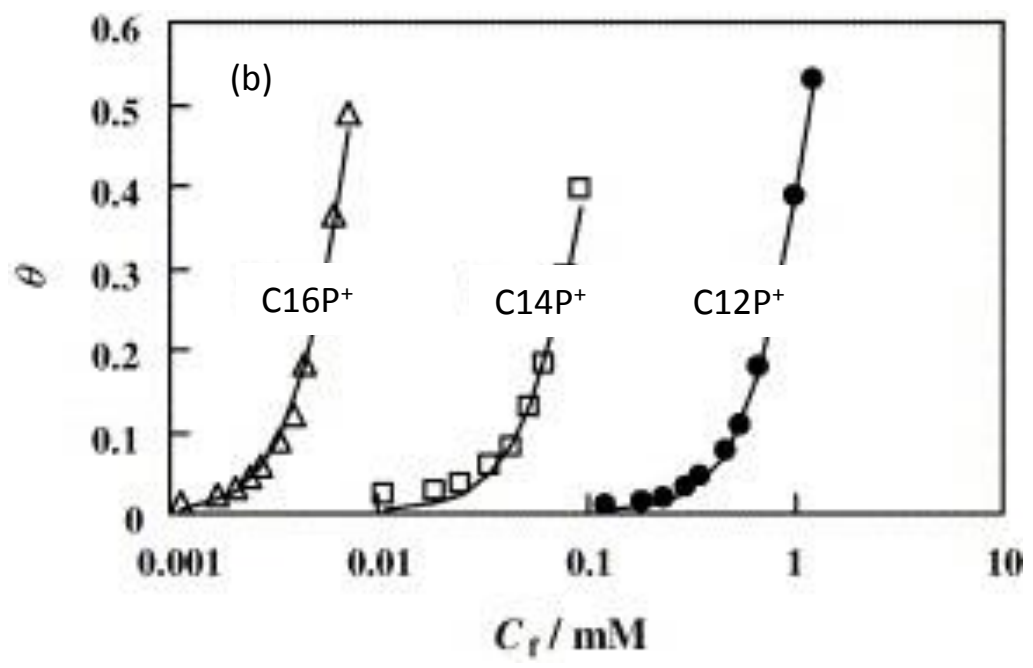



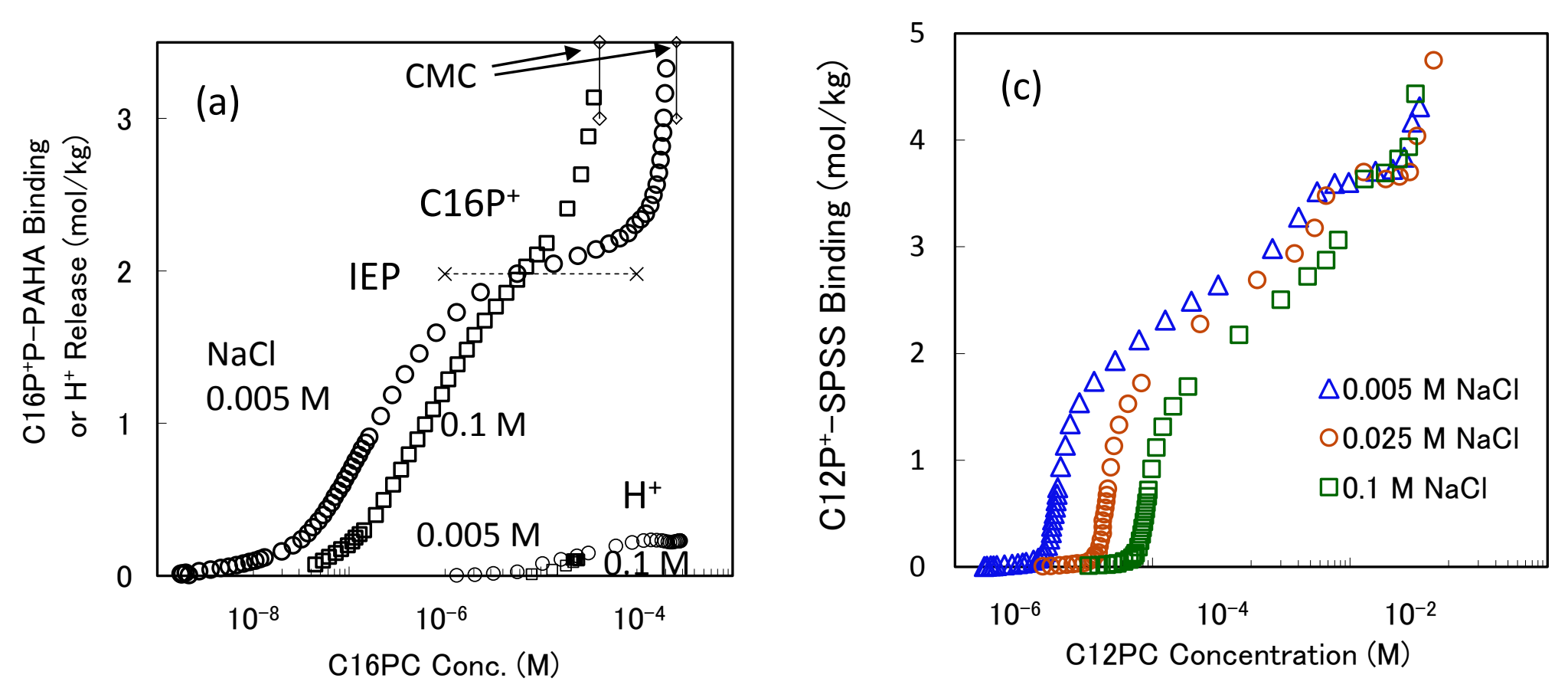

Fig. 10.
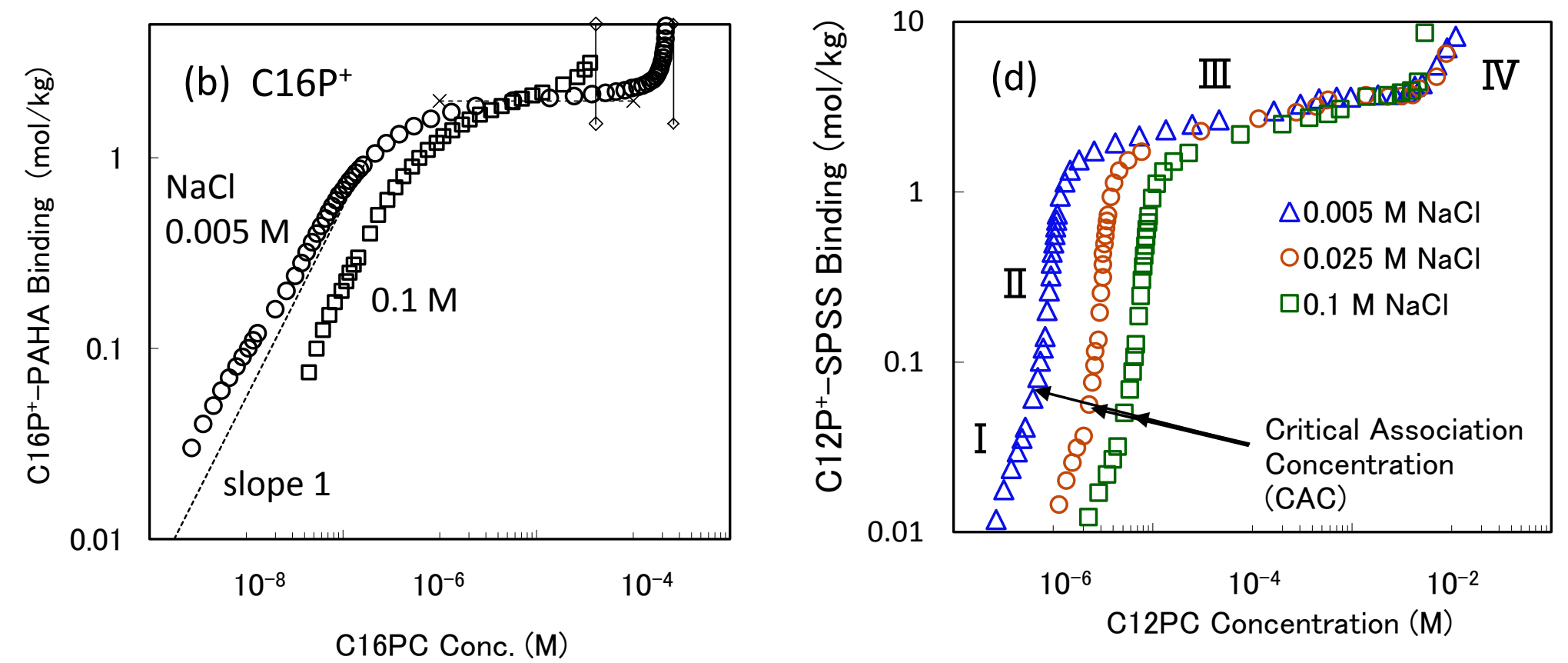


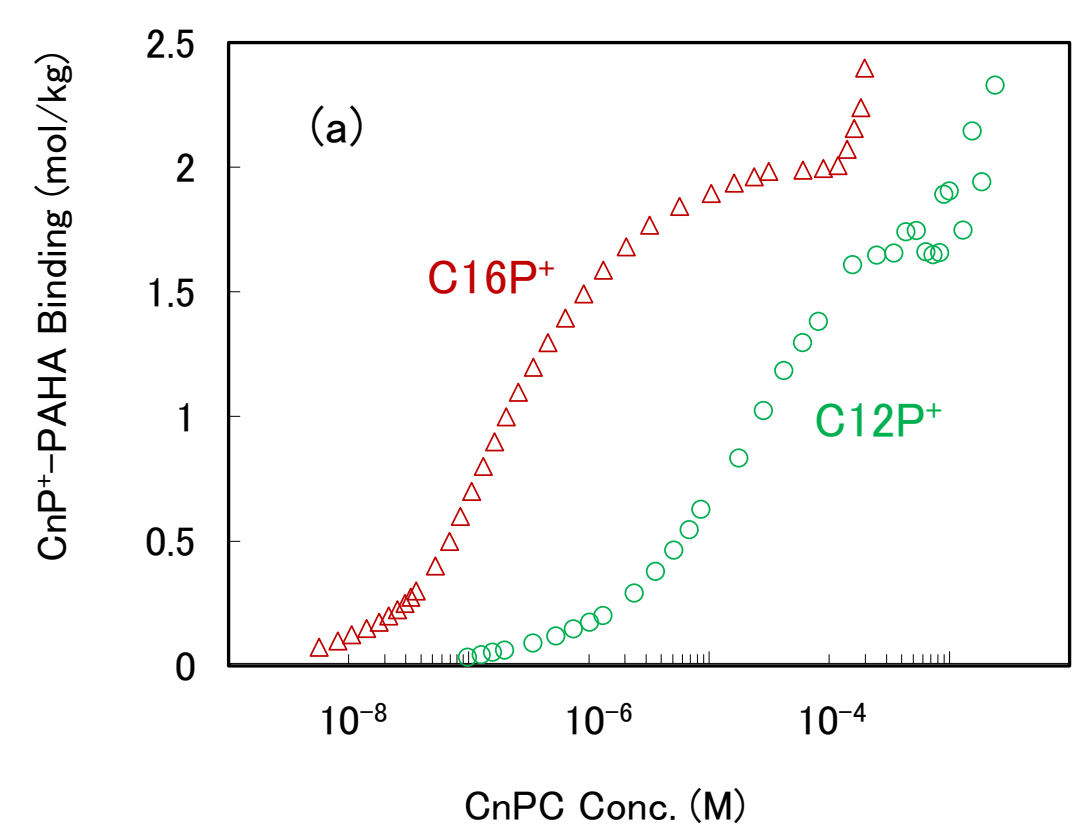

Fig. 11.

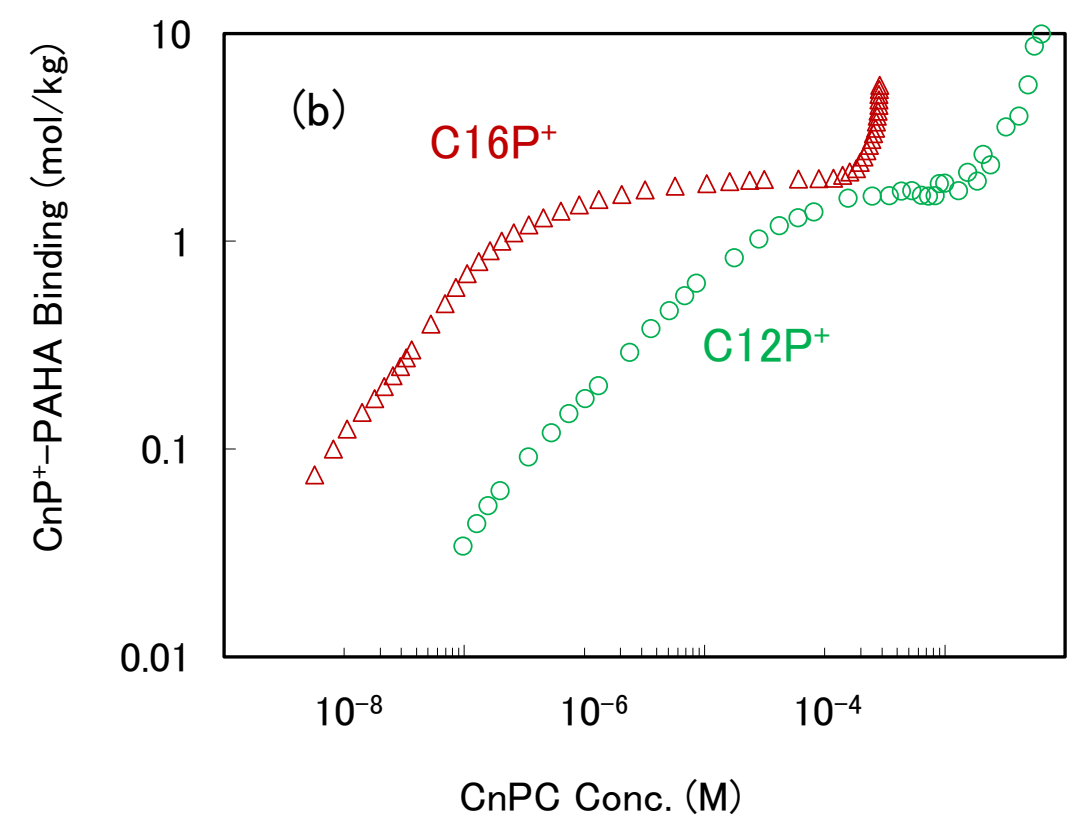




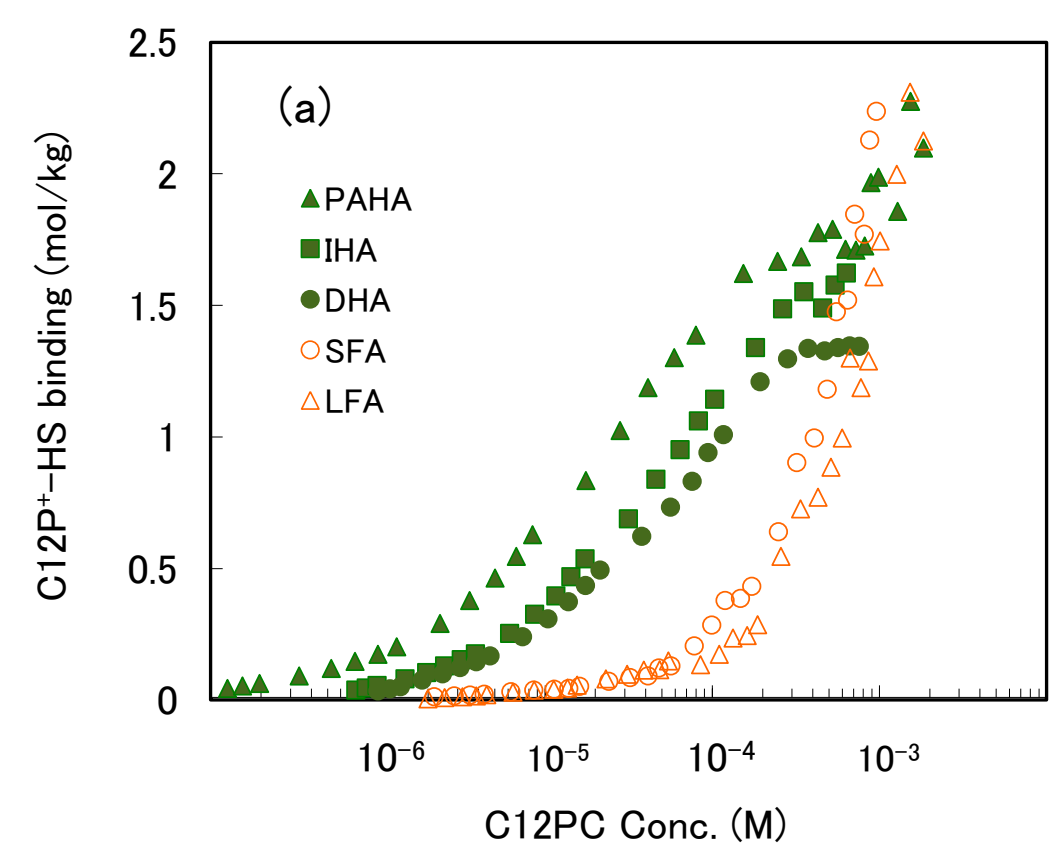

Fig. 12.

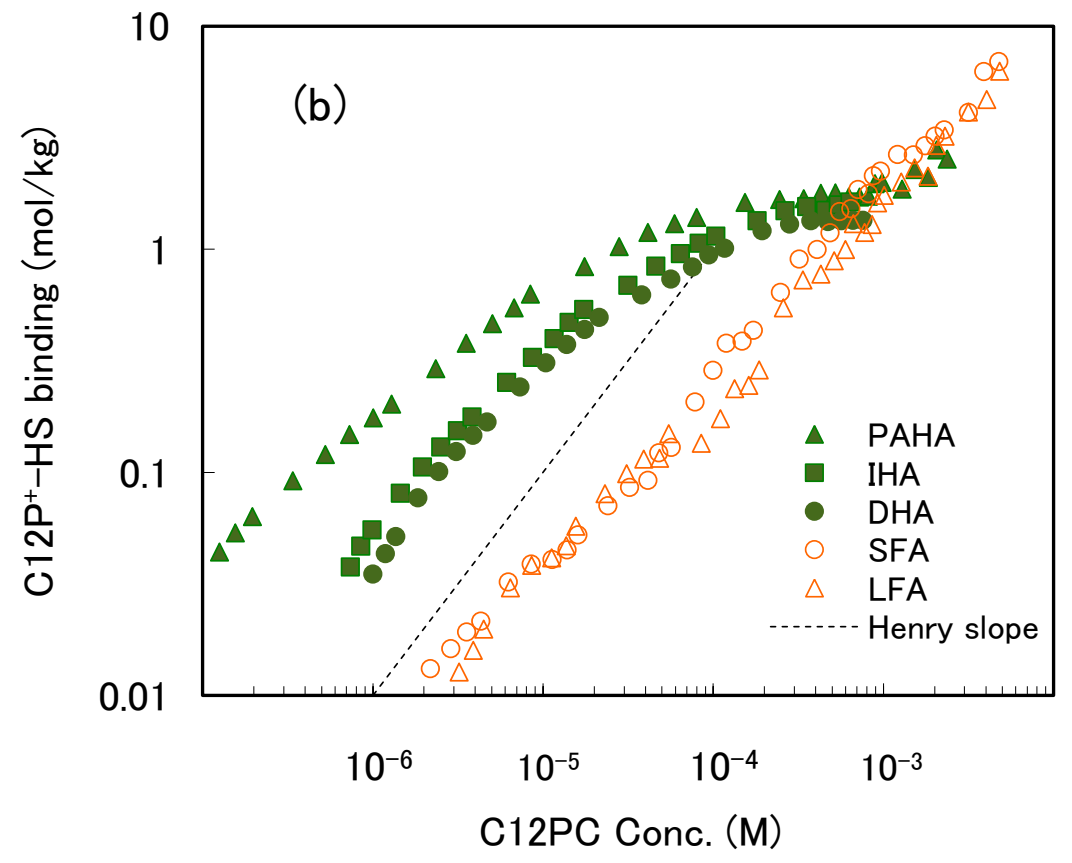




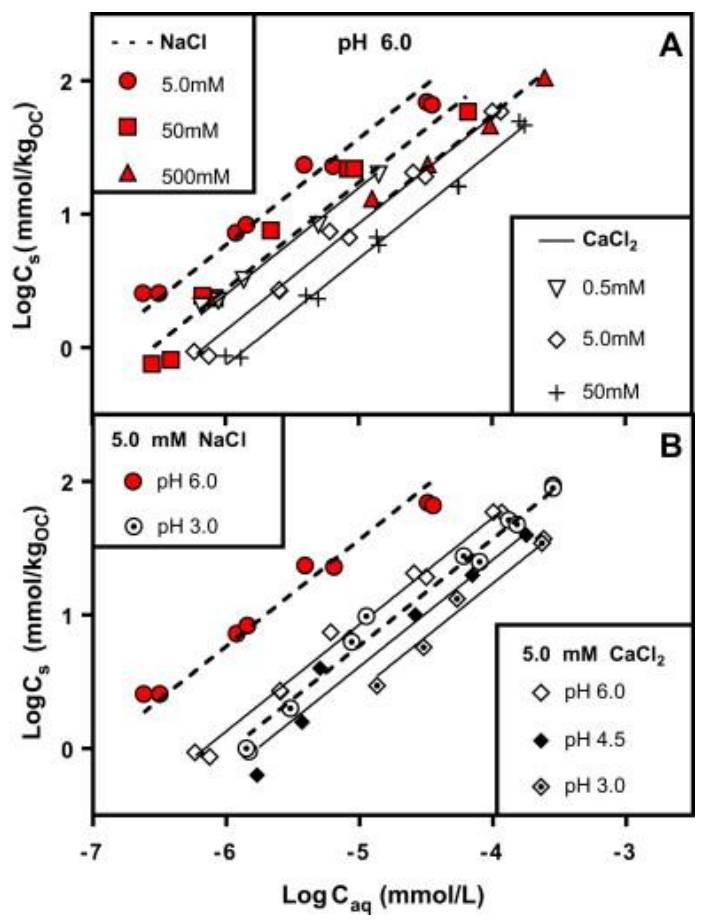

Fig. 13. 


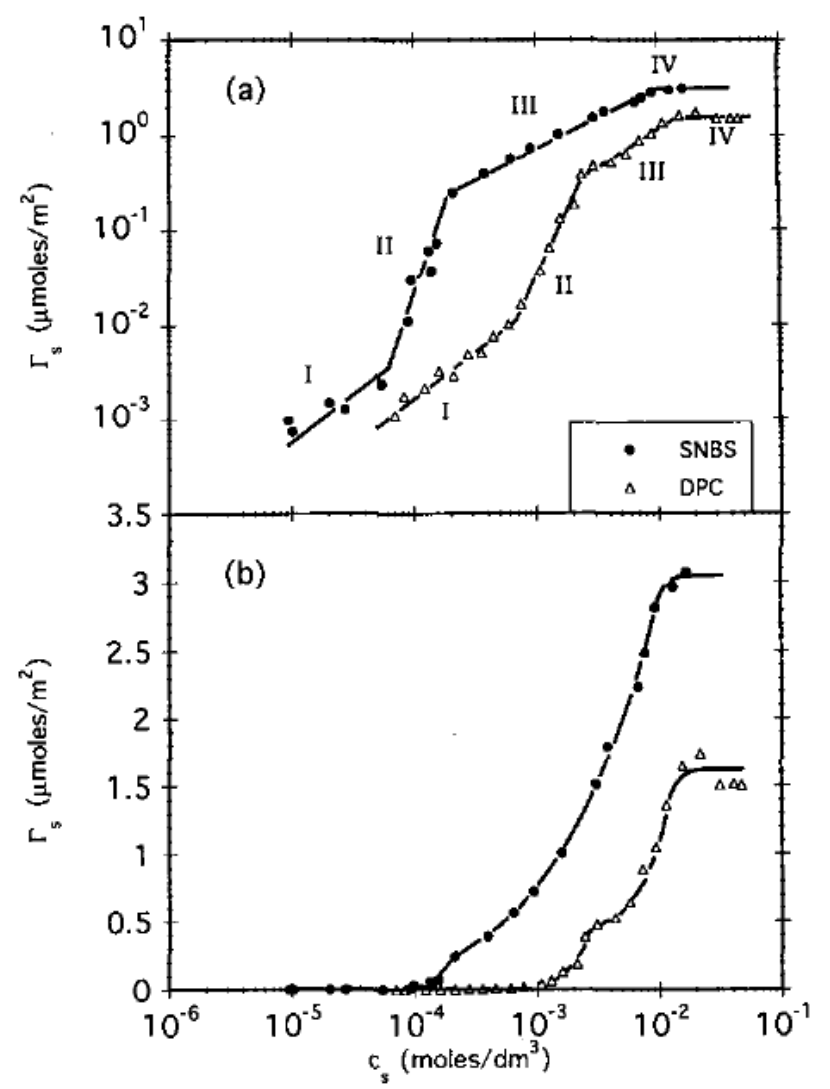

Fig. 14. 


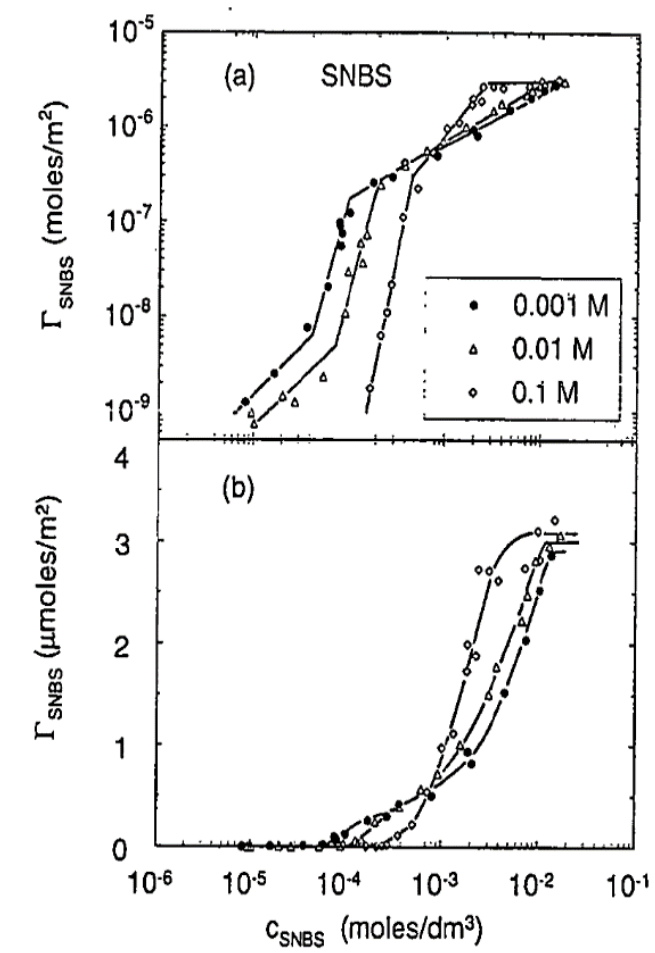

Fig. 15. 
Fig. 16.

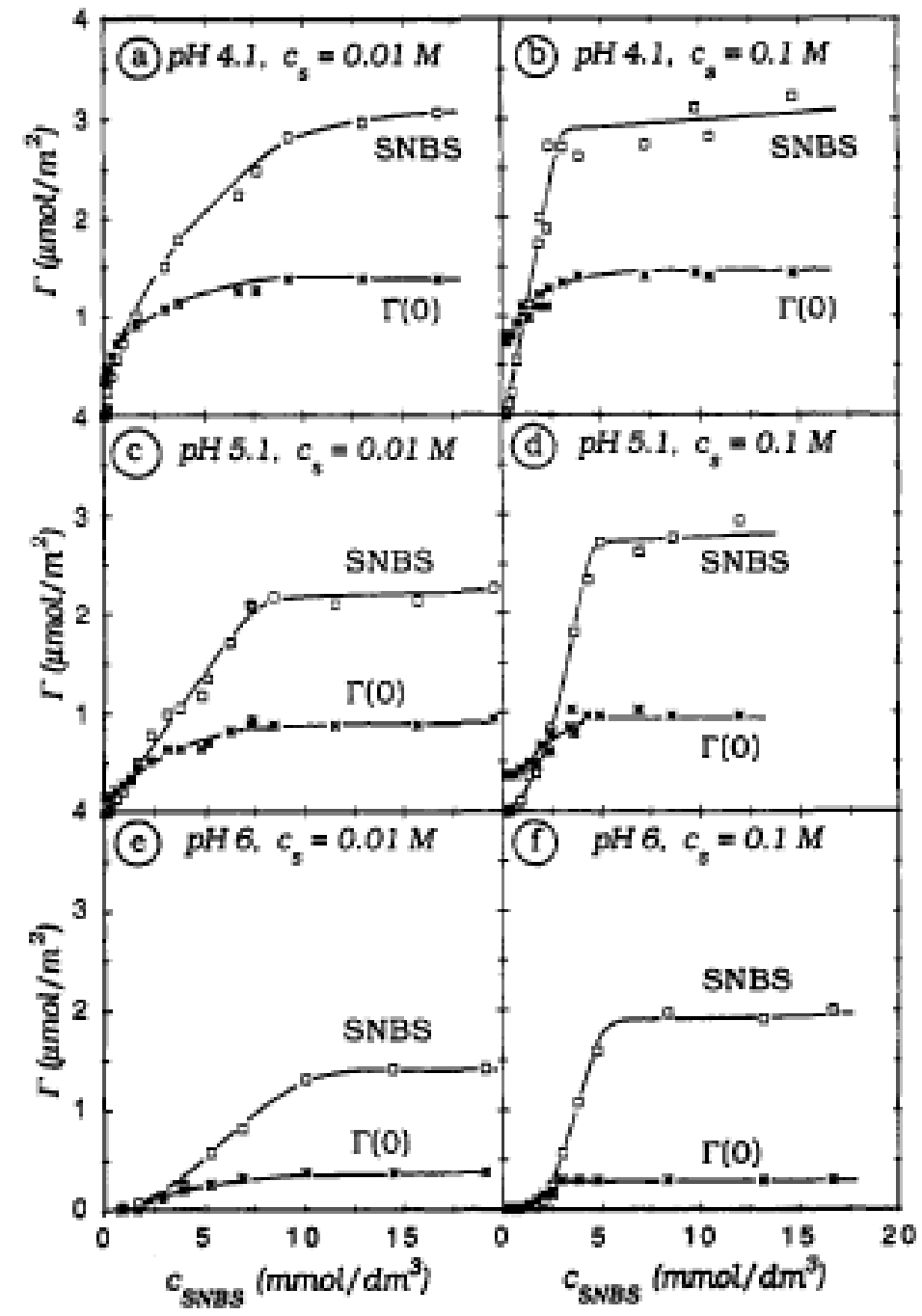




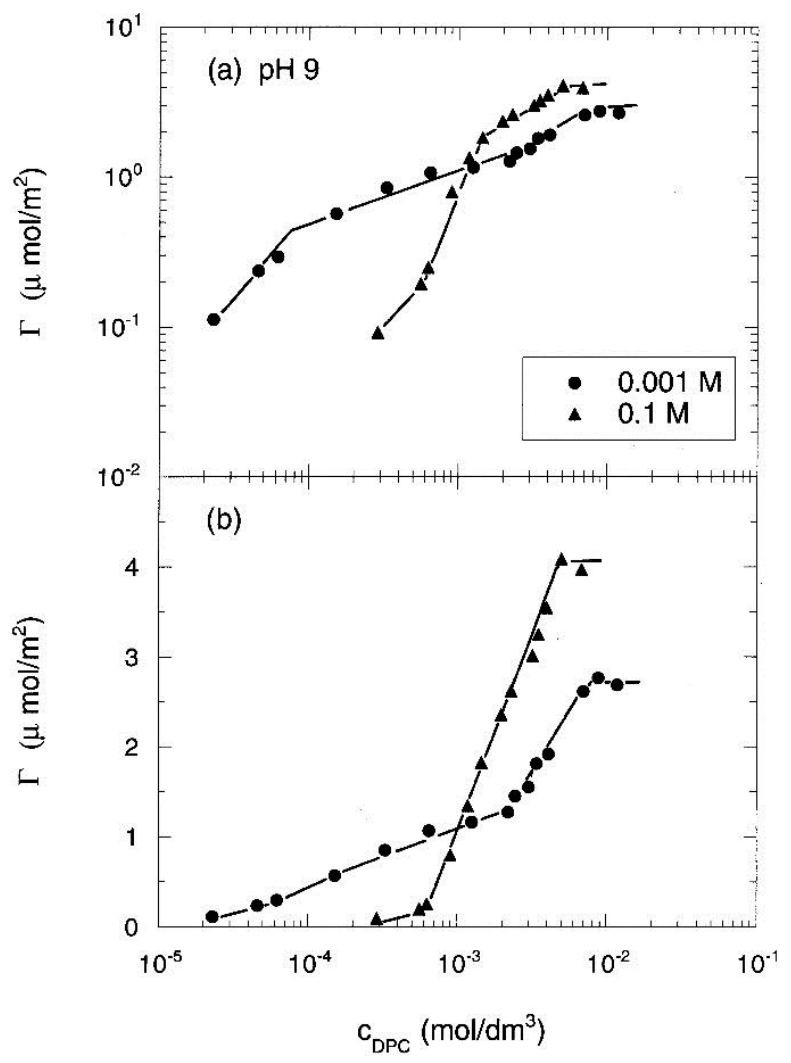

Fig. 17. 


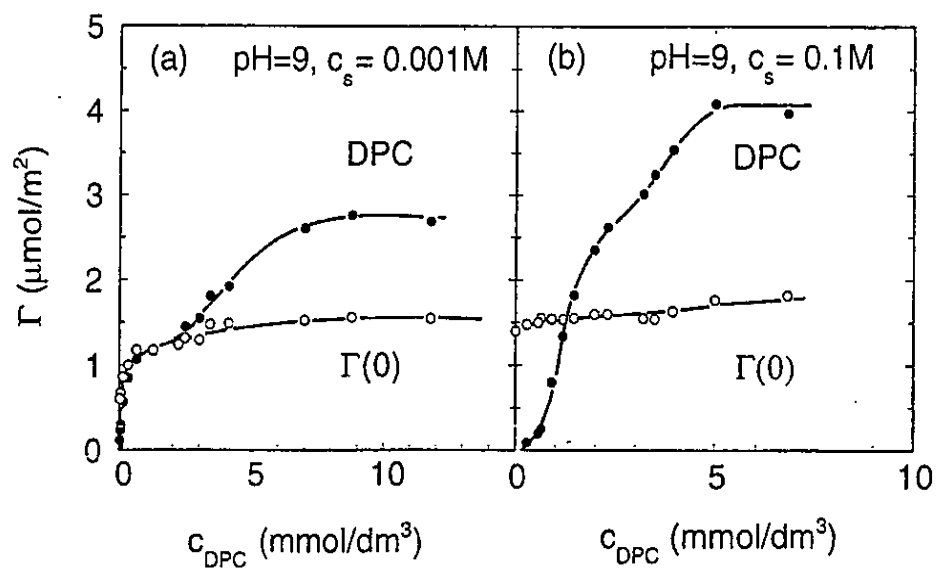

Fig. 18. 


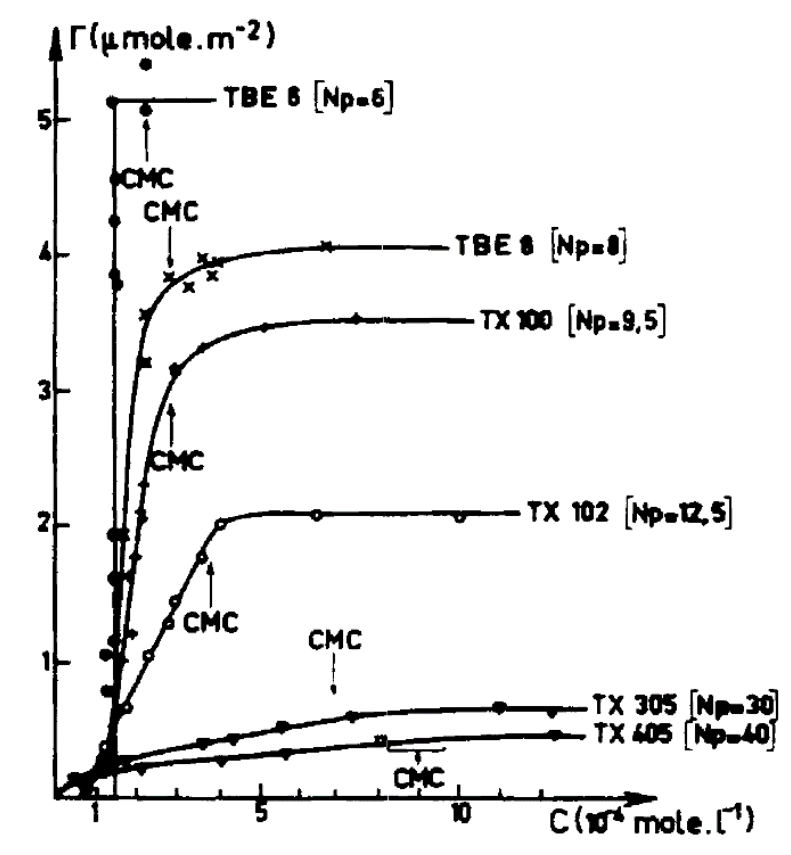

Fig. 19. 


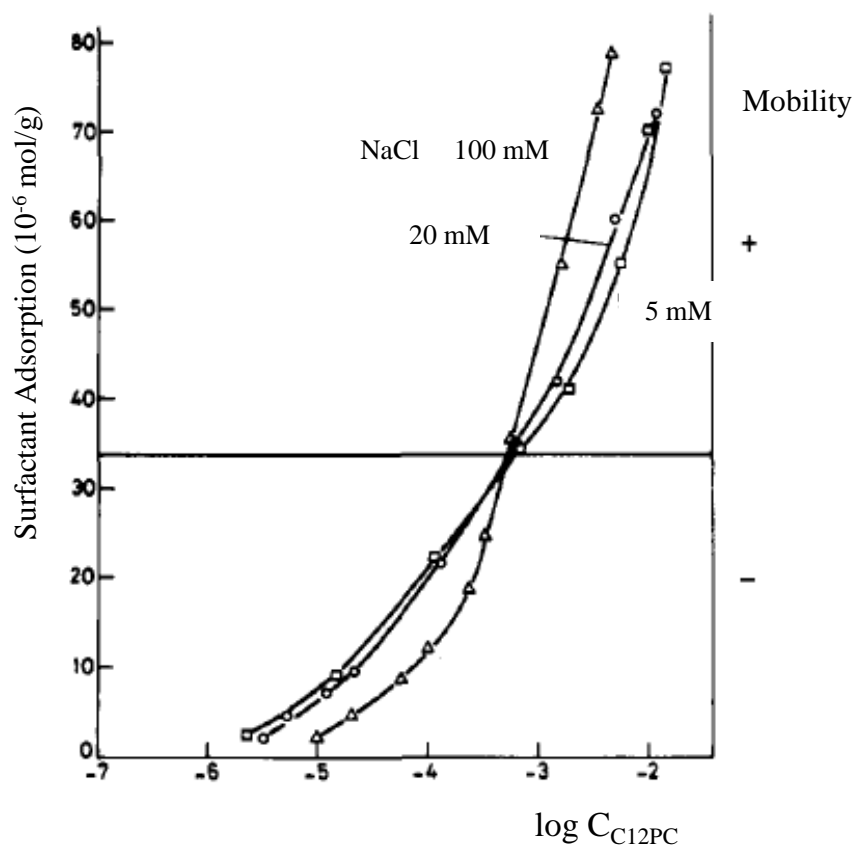

Fig. 20. 


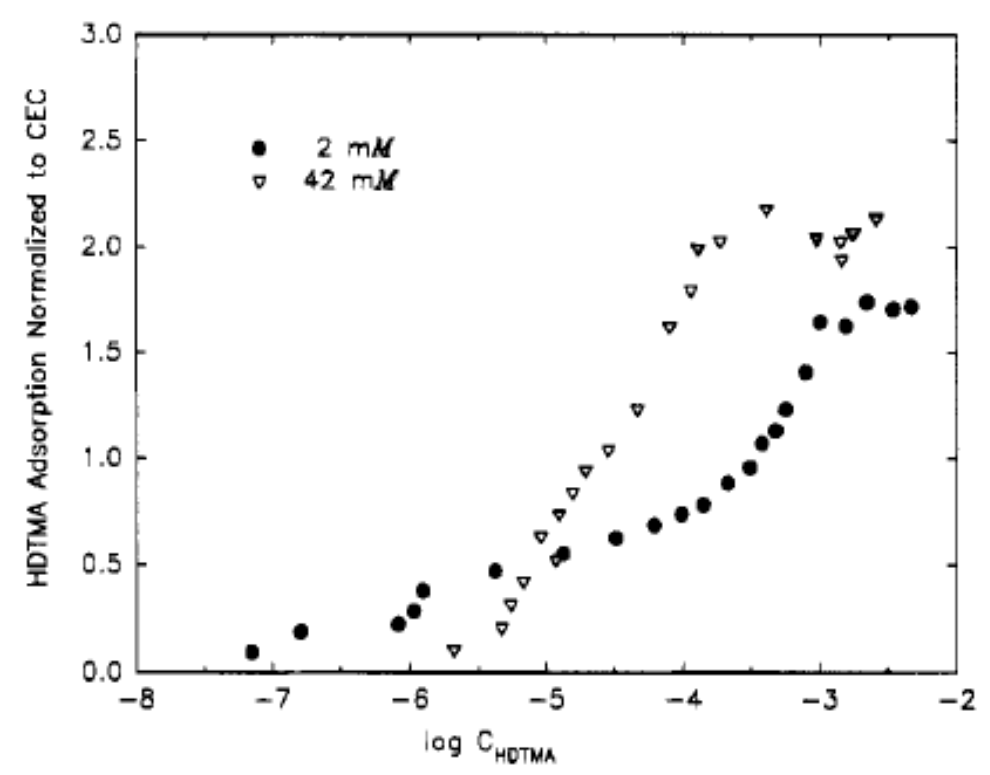

Fig. 21. 


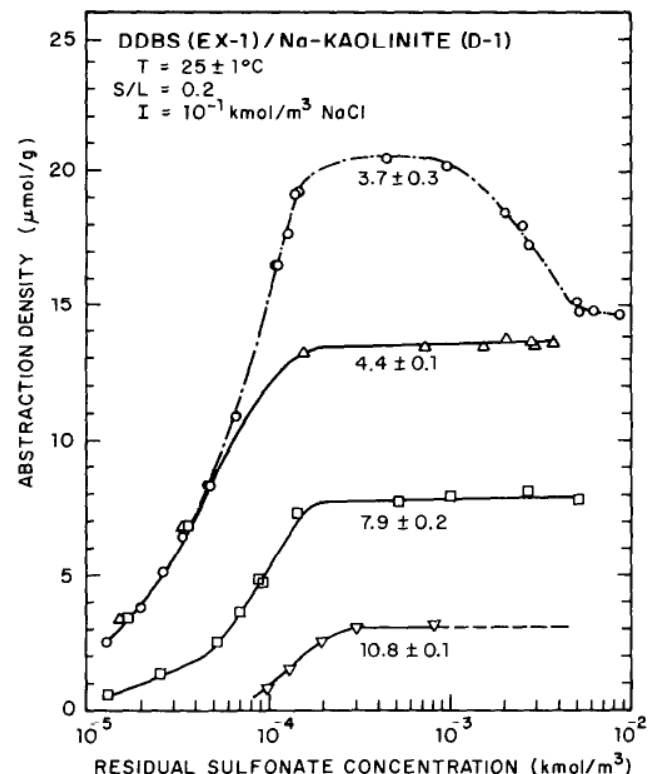

Fig. 22. 


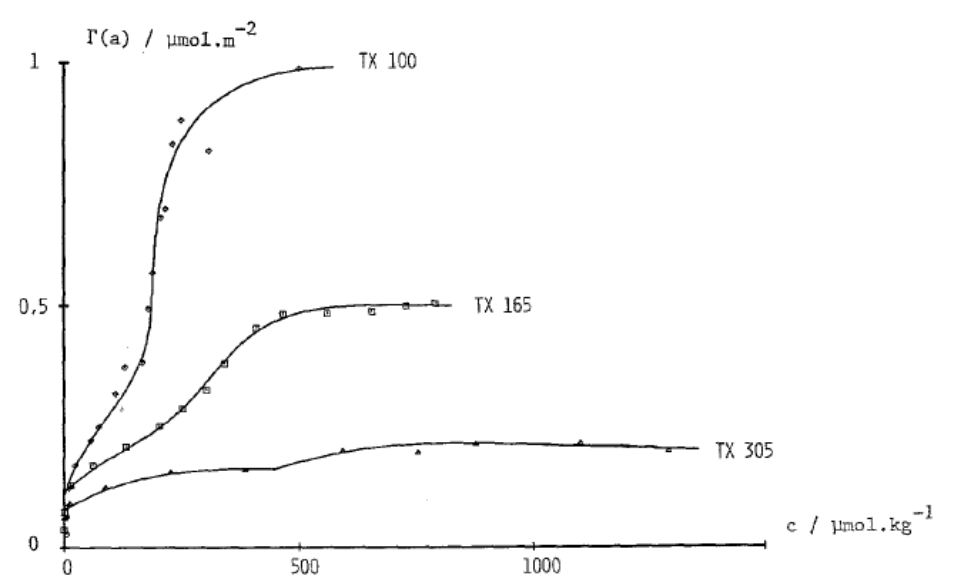

Fig. 23. 


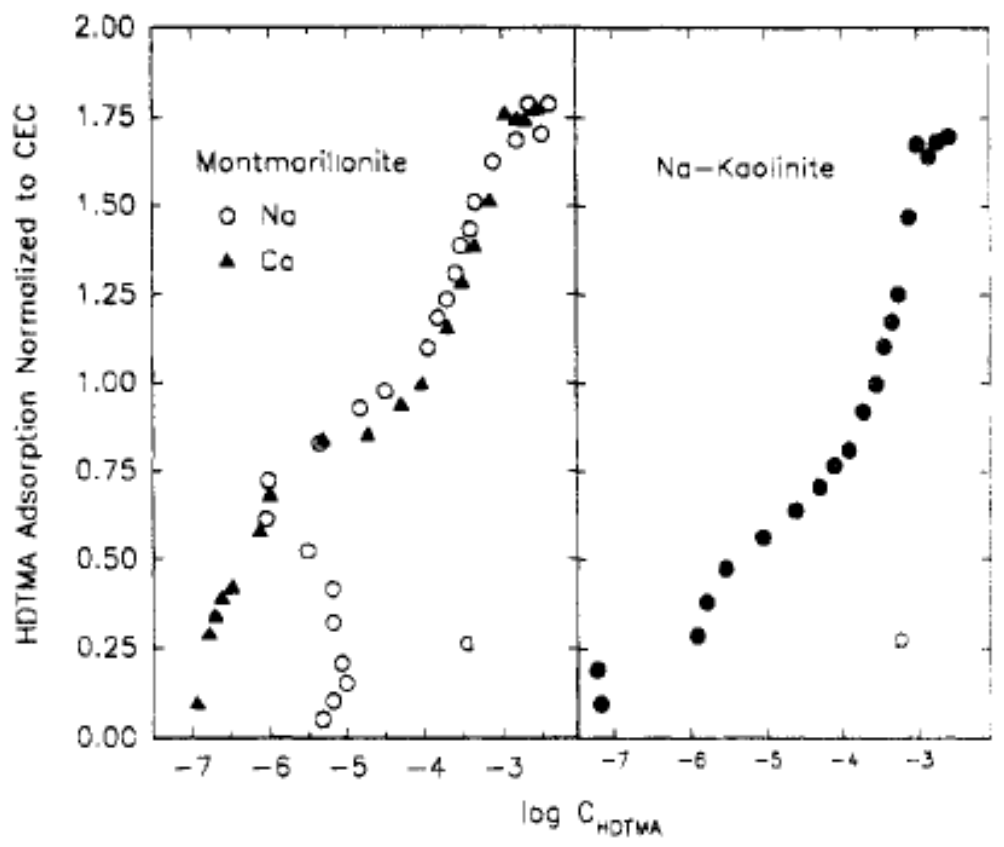

Fig. 24. 

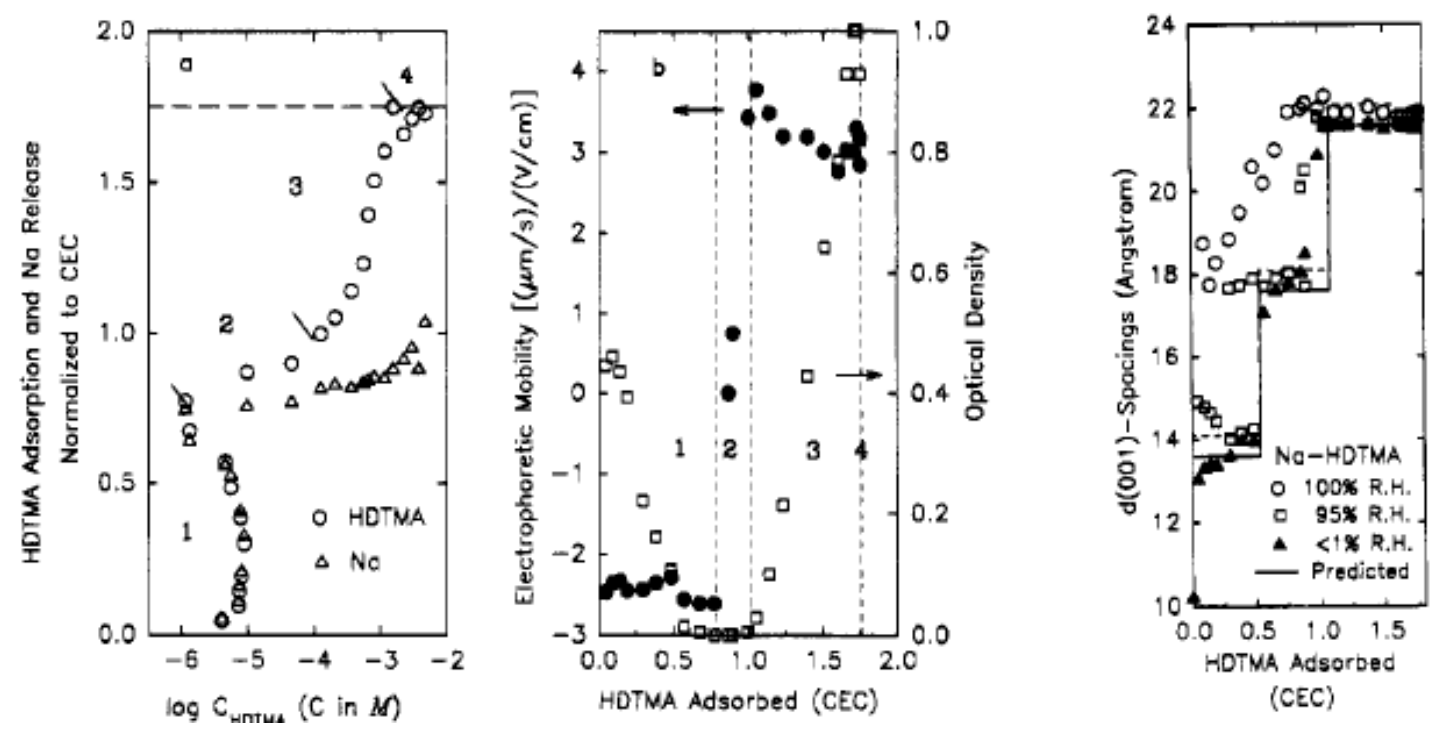

Fig. 25. 

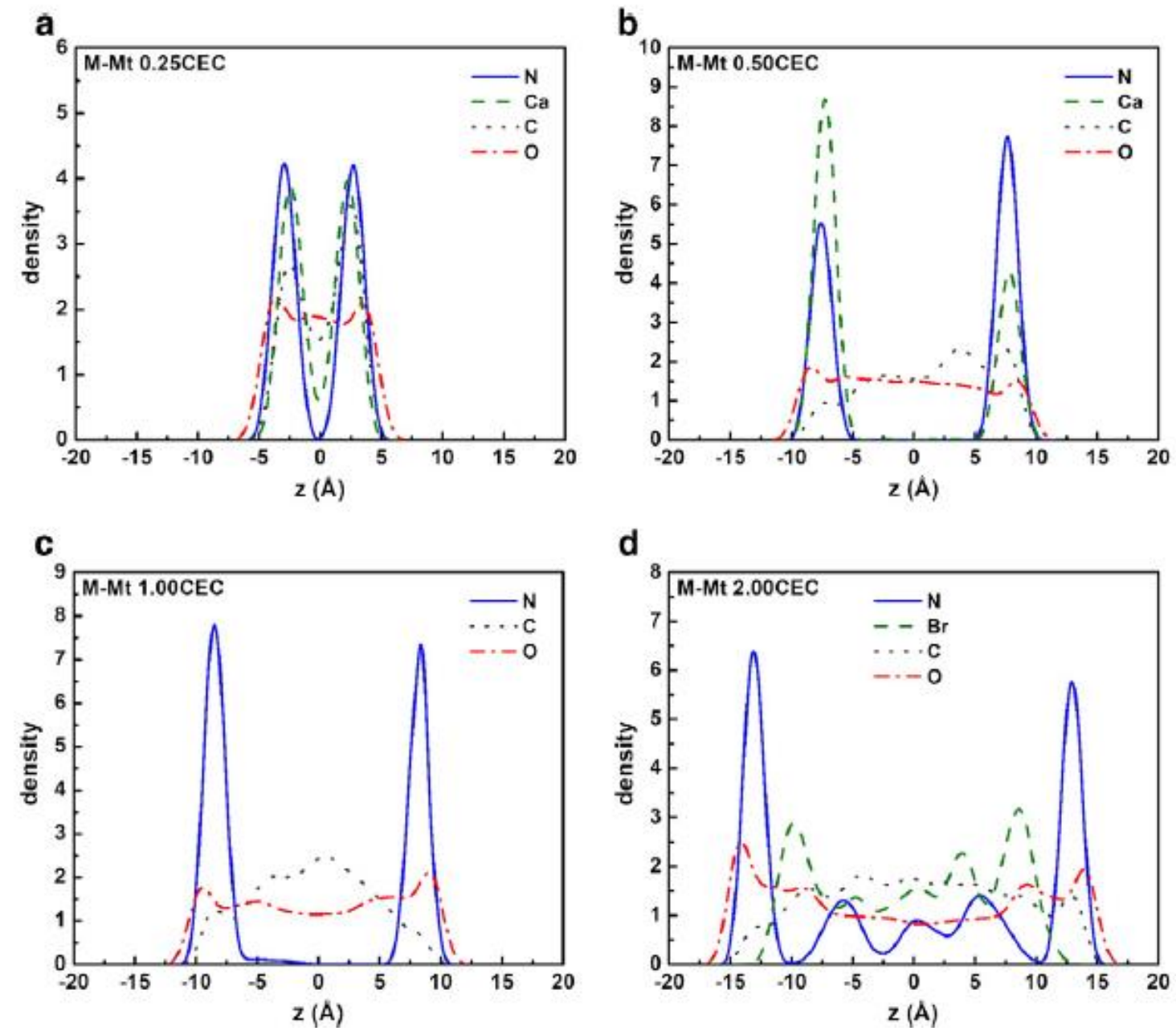

Fig. 26. 
b

7007.

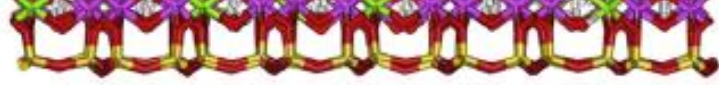

a

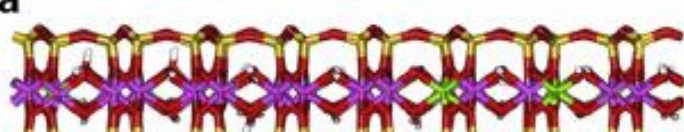
$200000000=$

28 o

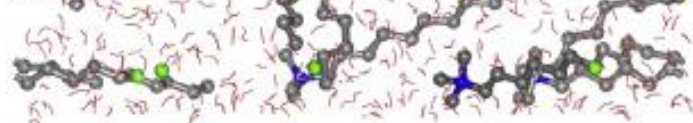

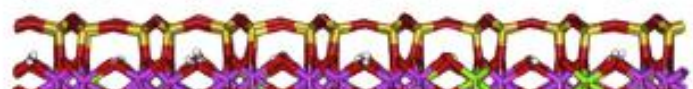

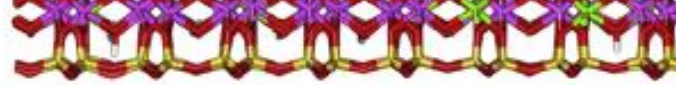

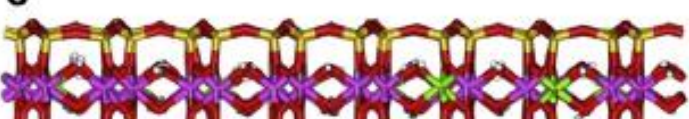

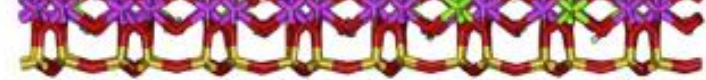

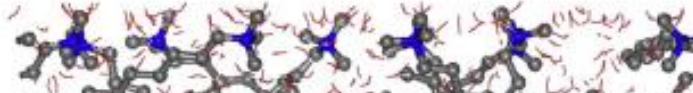
- 5 सी 52 o

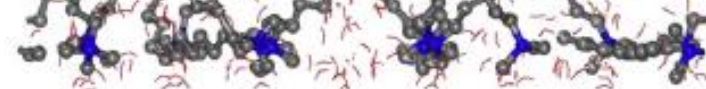
moñonomonoma

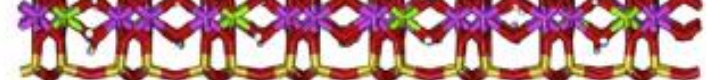

Fig. 27.

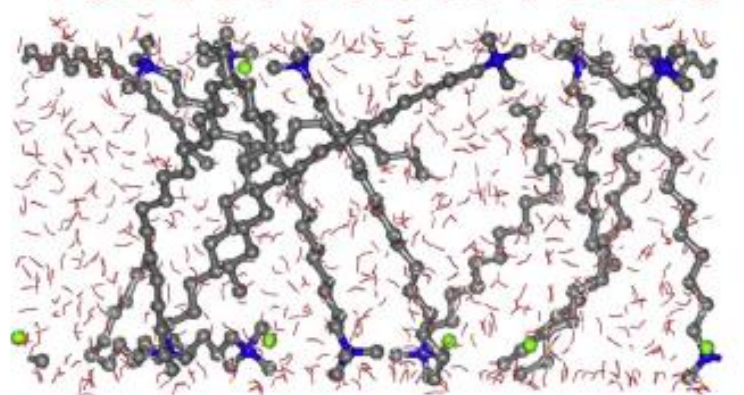

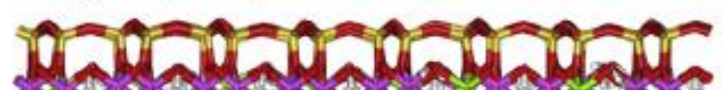

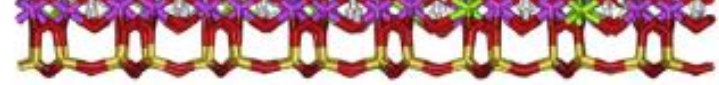

d

กด

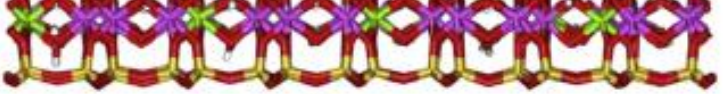

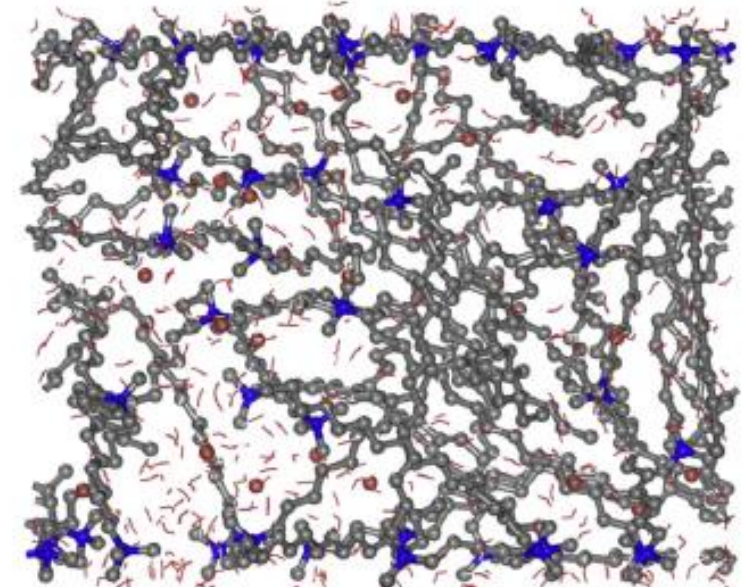

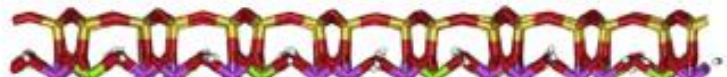

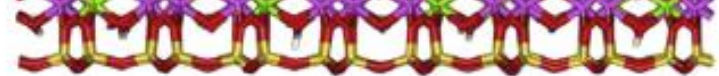




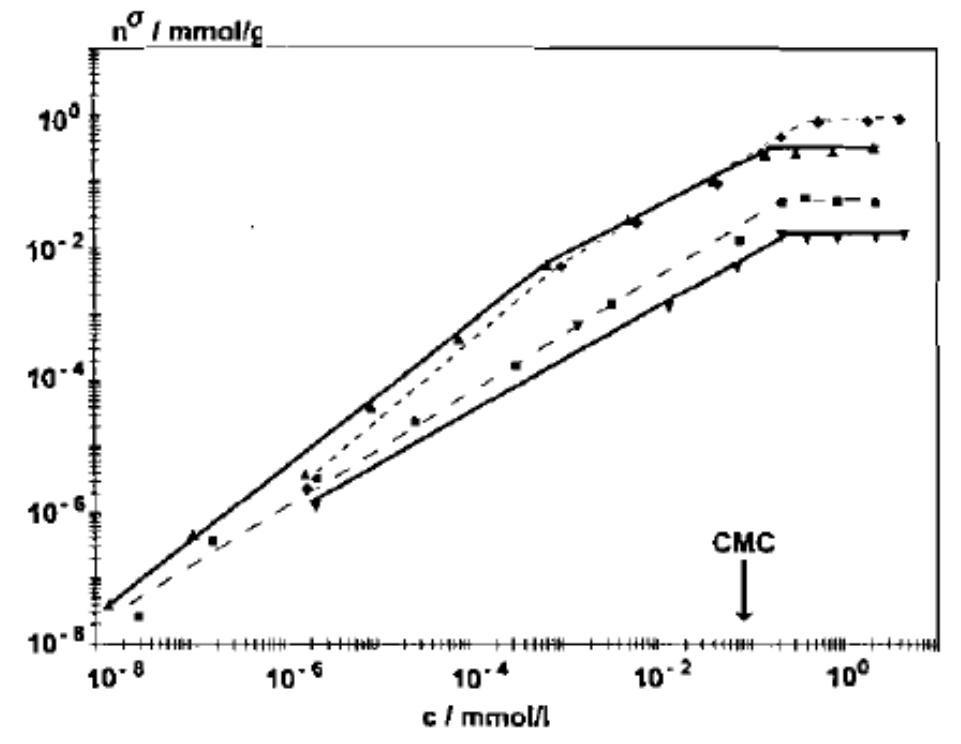

Fig. 28. 


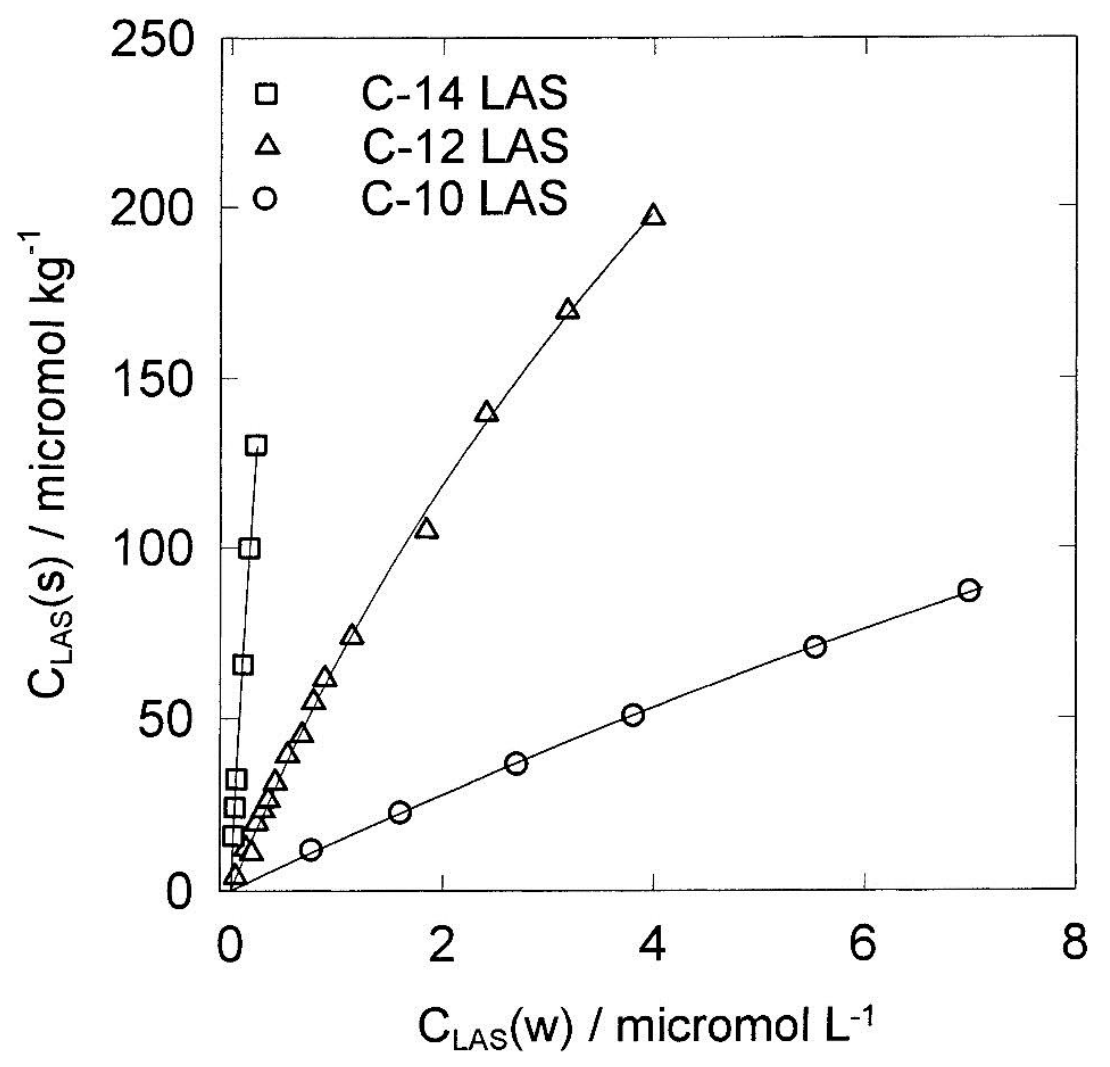

Fig. 29 


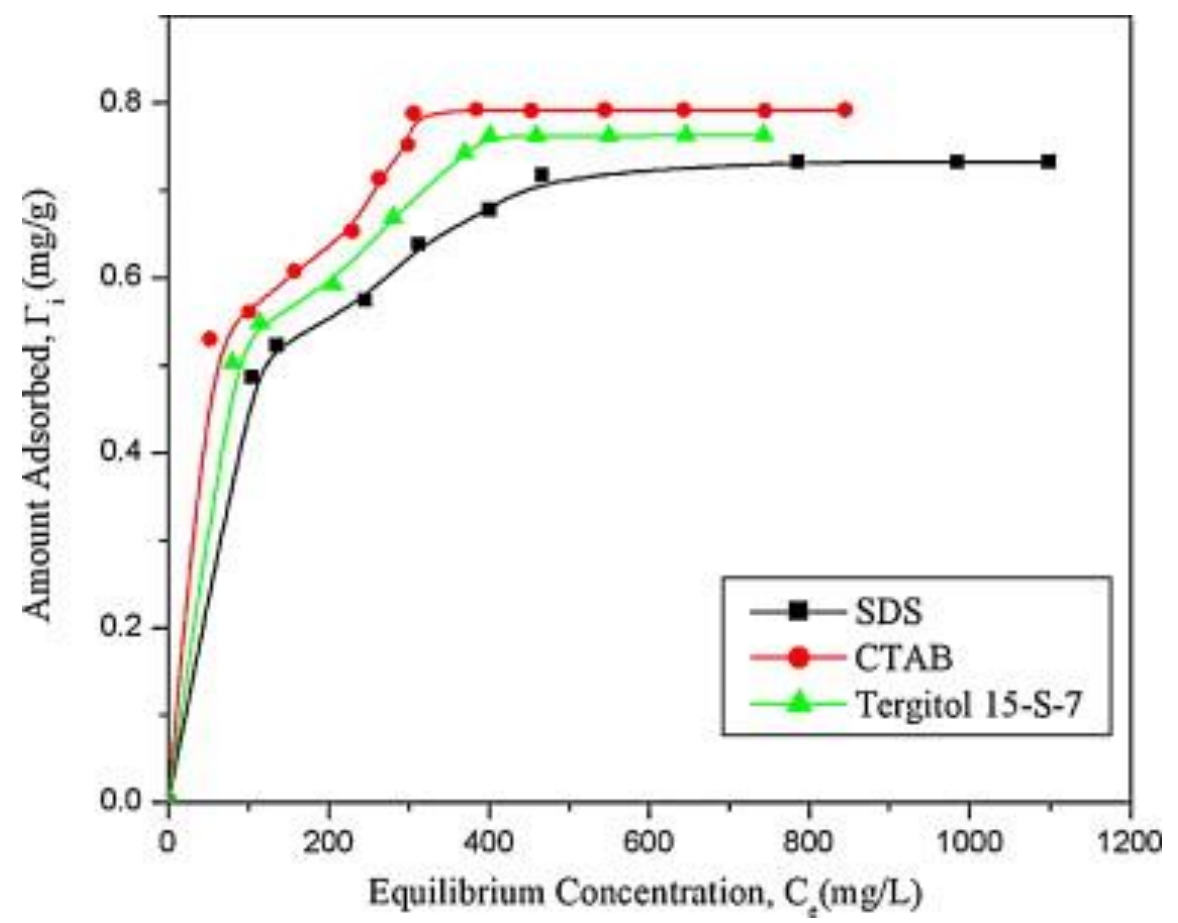

Fig. 30 


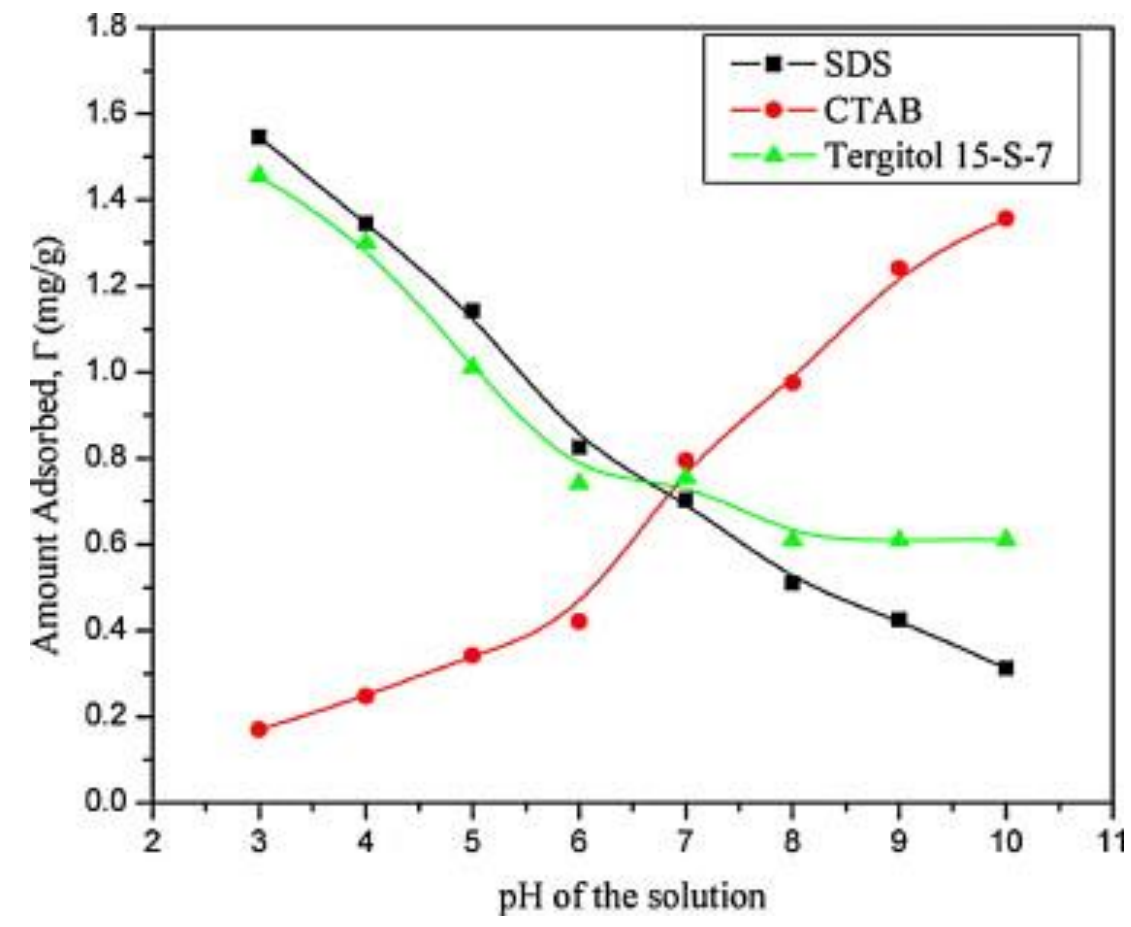

Fig. 31 

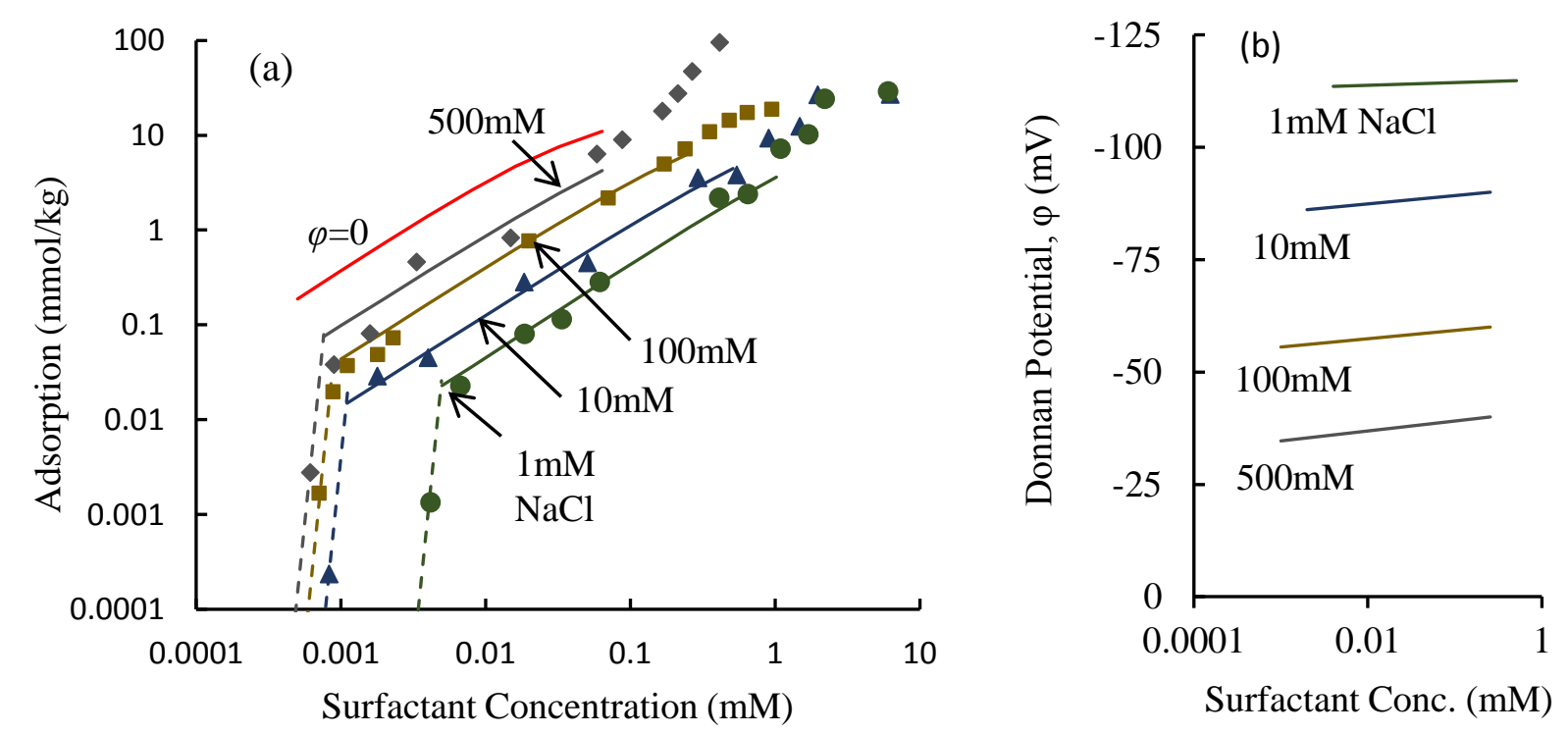

Fig. 32 


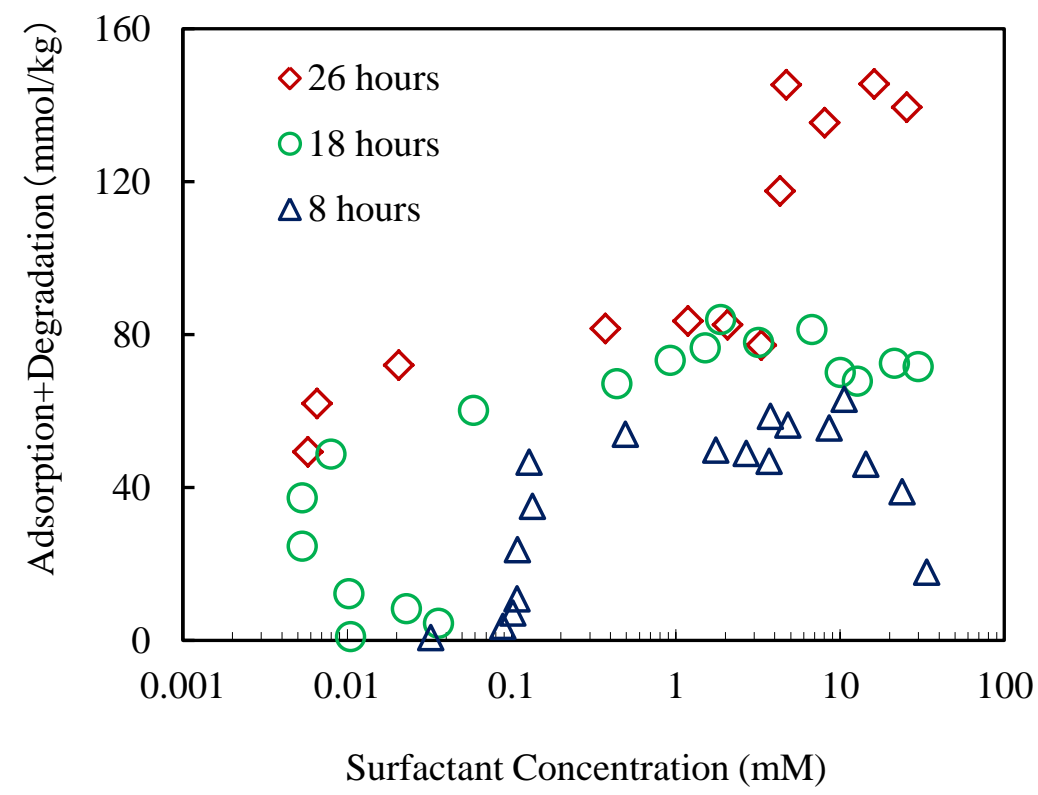

Fig. 33 

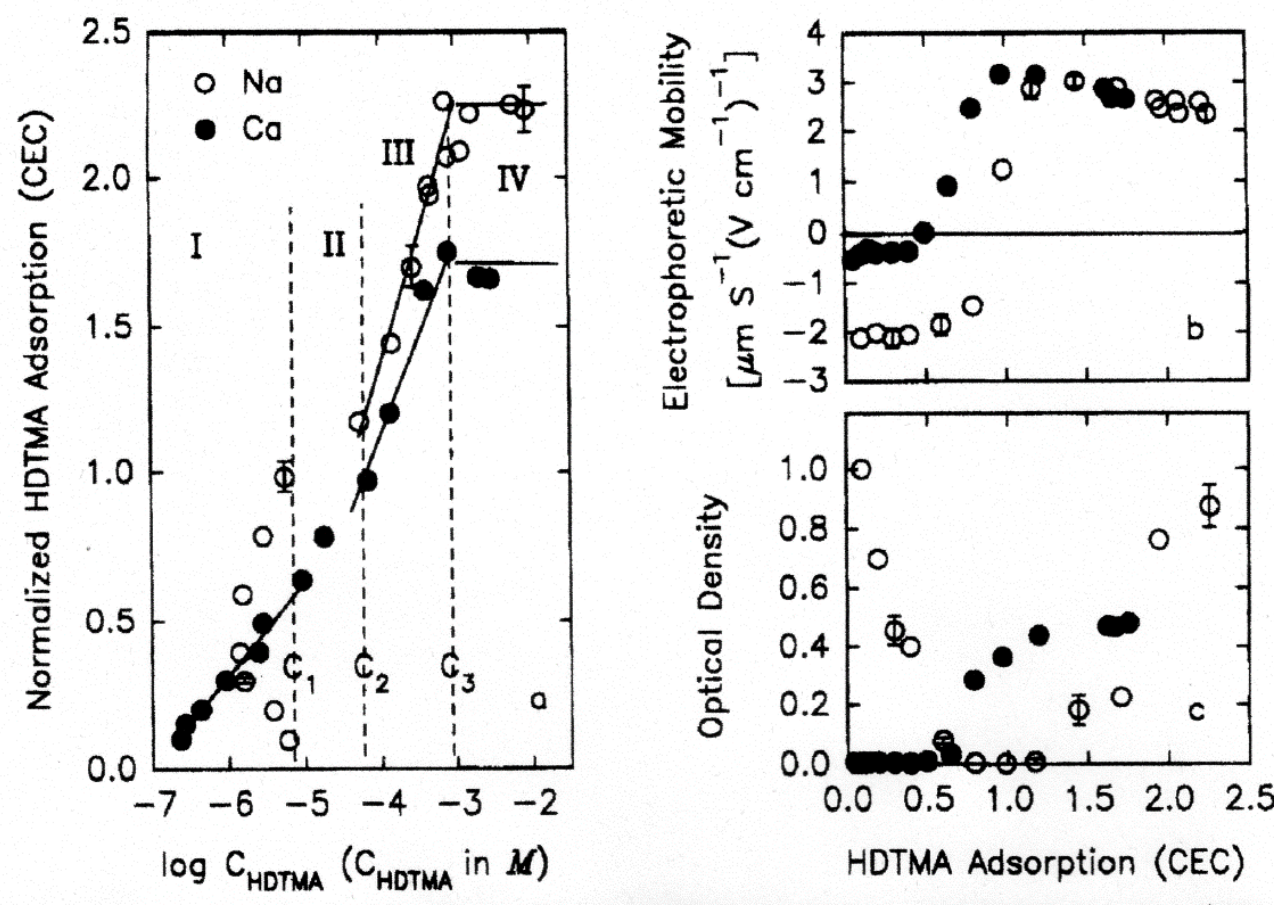

Fig. 34 

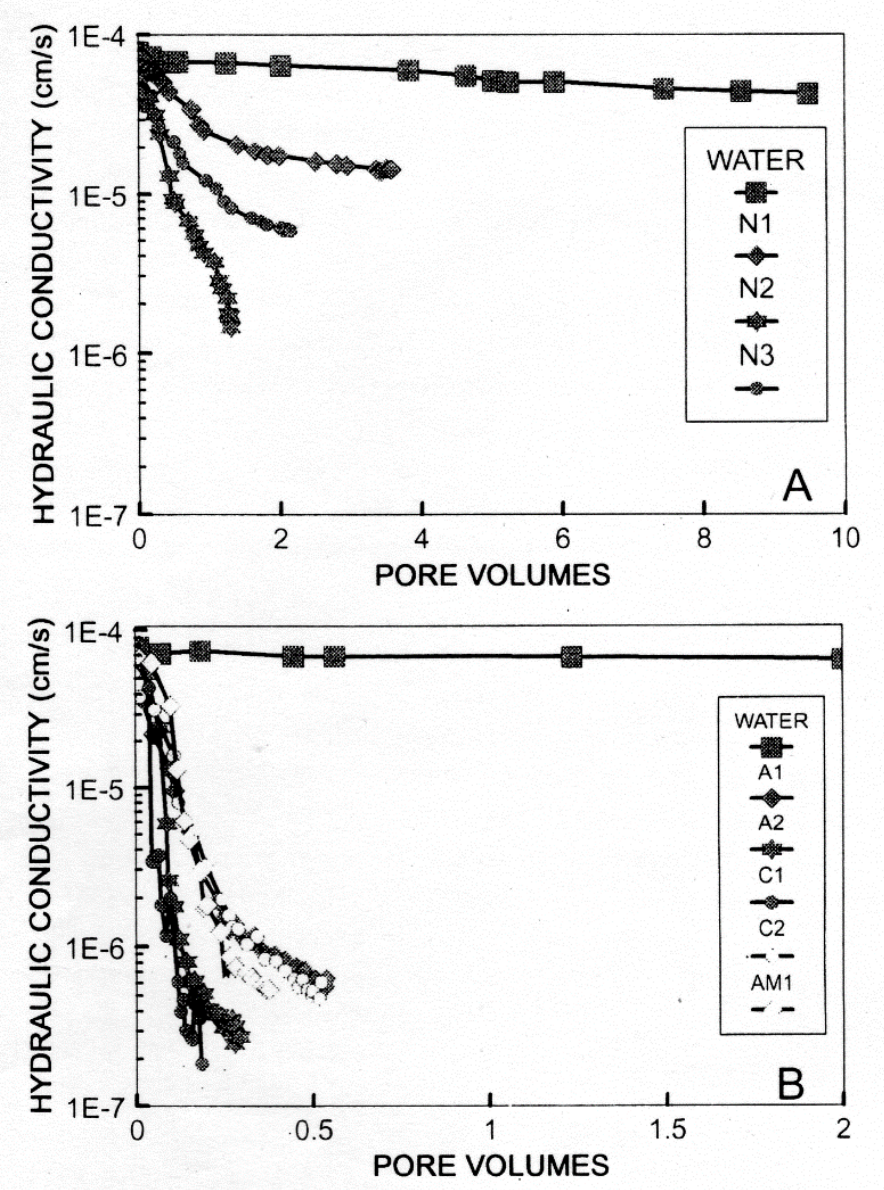

Fig. 35 


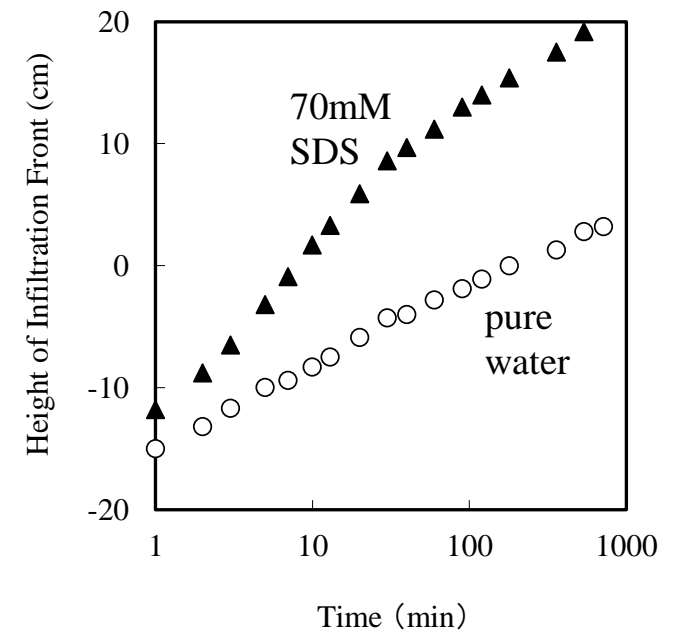

Fig. 36 